\title{
THE $W, Z$ SCALE FUNCTIONS KIT FOR FIRST PASSAGE PROBLEMS OF SPECTRALLY NEGATIVE LÉVY PROCESSES, AND APPLICATIONS TO CONTROL PROBLEMS
}

\author{
${\text { Florin } \text { Avram }^{1, *}, \text { Danijel Grahovac }}^{2}$ and Ceren Vardar-Acar ${ }^{3}$
}

\begin{abstract}
In the last years there appeared a great variety of identities for first passage problems of spectrally negative Lévy processes, which can all be expressed in terms of two " $q$-harmonic functions" (or scale functions) $W$ and $Z$. The reason behind that is that there are two ways of exiting an interval, and thus two fundamental "two-sided exit" problems from an interval (TSE). Since many other problems can be reduced to TSE, researchers developed in the last years a kit of formulas expressed in terms of the " $W, Z$ alphabet". It is important to note - as is currently being shown - that these identities apply equally to other spectrally negative Markov processes, where however the $W, Z$ functions are typically much harder to compute. We collect below our favorite recipes from the Lévy " $W, Z$ kit", drawing from various applications in mathematical finance, risk, queueing, and inventory/storage theory. A small sample of applications concerning extensions of the classic de Finetti dividend problem is offered. An interesting use of the kit is for recognizing relationships between problems involving behaviors apparently unrelated at first sight (like reflection, absorption, etc.). Another is expressing results in a standardized form, improving thus the possibility to check when a formula is already known.
\end{abstract}

Mathematics Subject Classification. 60G51, 60G40, 60J45.

Received November 1, 2017. Accepted November 6, 2019.

\section{INTRODUCTION}

From our biased point of view, the $W, Z$ scale functions kit is a new set of clothes for the classic first passage theory used in risk, queueing, mathematical finance and related fields, which was developed over the last 40 yr. A recent explosion of new contributions to this topic, notably to processes with Parisian ruin and reflection see Section 8, and the extension to spectrally negative Markov processes - see Section 11, suggested the utility of offering a new review. We attempted to pack in our "cookbook" a possibly overwhelming quantity of results; the best way for the reader to get an idea of what's to be found here might be to have first a quick look at the List of notations Section 13.

In this section we introduce the Cramér-Lundberg risk process, we define first passage times and some main quantities of interest for the control and optimization of risk processes.

Keywords and phrases: Spectrally negative processes, scale functions, Gerber-Shiu functions, Skorokhod regulation, dividend optimization, capital injections, processes with Poissonian/Parisian observations, generalized drawdown stopping.

${ }^{1}$ Laboratoire de Mathématiques Appliquées, Université de Pau, France.

2 Department of Mathematics, University of Osijek, Croatia.

3 Department of Statistics, Middle East Technical University, Ankara, Turkey.

* Corresponding author: florin.avram@orange.fr 
Origins. The origins of our field lie in the ruin problem for the Cramér-Lundberg or compound Poisson risk model $[1,109]$

$$
X_{t}=x-\left(\sum_{i=1}^{N_{t}^{(\lambda)}} C_{i}-c t\right)
$$

Here $c$ is the premium rate, $C_{i}, i=1,2, \ldots$ are i.i.d. nonnegative jumps with distribution $F(\mathrm{~d} z)$, arriving after independent exponentially distributed times with mean $1 / \lambda$, and $N^{(\lambda)}$ denotes the associated Poisson process counting the arrivals. Note that the process in parenthesis, called "cumulative loss", is used also to model the workload process of the $\mathrm{M} / \mathrm{G} / 1$ queue.

First passage theory concerns the first passage times above and below, and the hitting time of a level $b$. For any process $\left(X_{t}\right)_{t \geq 0}$, these are defined by

$$
\begin{aligned}
& T_{b,+}=T_{b,+}^{X}=\inf \left\{t \geq 0: X_{t}>b\right\}, \\
& T_{b,-}=T_{b,-}^{X}=\inf \left\{t \geq 0: X_{t}<b\right\}, \\
& T_{\{b\}}=T_{\{b\}}^{X}=\inf \left\{t \geq 0: X_{t}=b\right\},
\end{aligned}
$$

with $\inf \emptyset=+\infty$. The upper script $X$ will be typically omitted, as well as the signs,+- , when they are clear from the context.

First passage times are important in the control of reserves/risk processes. The rough idea is that when below low levels $a$, reserves processes should be replenished at some cost, and when above high levels $b$, they should be partly invested to yield income - see for example [1] and, for most recent work, papers like [4, 7, 31, 78], etc.

The first quantity to be studied historically was the eventual ruin probability

$$
\Psi(x)=P_{x}\left[T_{0}<\infty\right]
$$

for the Cramér-Lundberg/compound Poisson risk model [1, 109]. Subsequently, first passage (or exit) problems were studied in mathematical finance (barrier options, American options - see for example [94]), in risk [1], queueing [13], storage theory [48, 150], in mathematical biology [133], and in many other applications. The typical approach for a long while consisted in taking Laplace transform of the associated Kolmogorov integro-differential equation involving the generator operator.

In recent years it became clear that most first passage problems for spectrally negative or spectrally positive Lévy processes may be reduced to the solution of the two fundamental "two-sided exit" problems from an interval (TSE), upwards or downwards. At their turn, these can be ergonomically expressed in terms of two scale functions $/ q$-harmonic functions $W_{q}(x), Z_{q}(x, \theta)$. In the case of spectrally negative processes, one ends up with the following equations: ${ }^{1}$

$$
\begin{aligned}
\bar{\Psi}_{q}^{b}(x, a) & :=\mathbb{E}_{x}\left[e^{-q T_{b,+}} \mathbb{1}_{\left\{T_{b,+}<T_{a,-}\right\}}\right]=\frac{W_{q}(x-a)}{W_{q}(b-a)}, q \geq 0, a \leq x \leq b, \\
\Psi_{q, \theta}^{b}(x, a) & :=\mathbb{E}_{x}\left[e^{-q T_{a,-}+\theta\left(X_{\left.T_{a,-}-a\right)}\right.} \mathbb{1}_{\left\{T_{a,-}<T_{b,+}\right\}}\right]=Z_{q}(x-a, \theta)-W_{q}(x-a) \frac{Z_{q}(b-a, \theta)}{W_{q}(b-a)}, \theta \geq 0 .
\end{aligned}
$$

We will call $\bar{\Psi}_{q}^{b}(x, a), \Psi_{q}^{b}(x, a)$ (killed) survival and ruin first passage probabilities, respectively. When $a=0$, it will be omitted, to simplify the notation.

\footnotetext{
${ }^{1}$ The first equation generalizes the famous "gambler's winning" formula for the symmetric random walk $\bar{\Psi}_{0}^{b}(x, a)=$ $\mathbb{E}_{x}\left[\mathbb{1}_{\left\{T_{b,+}<T_{a,-}\right\}}\right]=\frac{x-a}{b-a}$.
} 
Remark 1.1. Note that the first quotient decomposition above holds true by the absence of positive jumps and by the strong Markov property, and that this defines $W_{q}$ up to a multiplicative constant. The second relation is equivalent to (1.11) below which defines $Z_{q}$ up to a multiplicative constant (see Thm. 12 in [78] and Rem. 6.4 below). For many other results in this vein, see $[22,32,42,43,78,85,94,104,142,153]$, and many other papers listed in the more detailed but still too succinct chronology in Section 12 below.

Remark 1.2. The relation between $W(x)$ and $\bar{\Psi}(x)$. When $q=0$, the scale function $W(x):=W_{0}(x)$ is related to the eventual ruin $\Psi(x)=P_{x}\left[T_{0}<\infty\right]$ and ultimate survival probabilities $\bar{\Psi}(x)=P_{x}\left[T_{0}=\infty\right]$, via

$$
\Psi(x)=1-\bar{\Psi}(x)=1-\kappa^{\prime}\left(0_{+}\right) W(x) .
$$

Here $\kappa$ is the Laplace exponent of $X$ given below in (2.1) and the Laplace transform of $W(x)$ is $\widehat{W}(s)=\frac{1}{\kappa(s)}$. Note that above and throughout the paper we will assume that $\kappa^{\prime}\left(0_{+}\right)$exists, which renders formulas simpler (and is typically satisfied in applications). (1.5) is related to the famous Pollaczek-Khinchine formula for the Laplace transform of the survival function of a spectrally negative Lévy process

$$
\widehat{\bar{\Psi}}(s):=\int_{0}^{\infty} e^{-s x} \bar{\Psi}(x) \mathrm{d} x=\frac{\kappa^{\prime}\left(0_{+}\right)}{\kappa(s)} .
$$

The scale function $W(x)$ provides an alternative characterization of a spectrally negative Lévy process, which may replace the classic Laplace exponent $\kappa(s)$.

Remark 1.3. The eventual ruin and survival probability have made the object of numerous numerical studies, for example by inversion of Padé approximations of $\frac{1}{\kappa(s)}[9,23,26]$ - see [1] for other methods and references. Furthermore, it is easy to adapt numerical studies of $W$ to yield $W_{q}$, by the so called Esscher transform (replacing $\kappa(s)$ by $\kappa(s+q)-\kappa(q))$ - see Remark 5.4. Note that once $W_{q}$ and $Z_{q}$ are computed, we have obtained also the answer to many other problems, thus removing the need for Laplace transform inversion. Hence, a cookbook of $W_{q}, Z_{q}$ formulas provides an alternative to the classic Markovian analytic approach.

Before continuing, we note that the last decade has witnessed also very interesting research on last passage times - see for example $[38,53,112,128]$. Since we had to stop at some point, these will not be covered in our review.

Control of dividends and capital injections. The next impetus came from control problems in risk theory which concern versions of $X_{t}$ which are reflected/constrained/regulated at first passage times (below or above):

$$
X_{t}^{[a}=X_{t}+L_{t}, \quad X_{t}^{b]}=X_{t}-U_{t}
$$

Here,

$$
\begin{aligned}
& L_{t}=L_{t}^{[a}=-\left(\underline{X}_{t}-a\right)_{-}, \quad \underline{X}_{t}=\inf _{0 \leq s \leq t} X_{s}, \\
& U_{t}=U_{t}^{b]}=\left(\bar{X}_{t}-b\right)_{+}, \quad \bar{X}_{t}:=\sup _{0 \leq s \leq t} X_{s},
\end{aligned}
$$

are the minimal "Skorokhod regulators" constraining $X_{t}$ to be bigger than $a$, and smaller than $b$, respectively, and we use the notation $x_{+}=\max (x, 0)$ and $x_{-}=\min (x, 0)$. 
One problem of historical interest is the de Finetti problem of expected total discounted dividends until the ruin time $T_{0,-}$, in the presence of a constant (reflecting ) dividend barrier - see (6.1). Interestingly, its solution

$$
V^{b]}(x)=\mathbb{E}_{x}\left[\int_{\left[0, T_{0,-}\right]} e^{-q t} \mathrm{~d} U_{t}\right]=\frac{W_{q}(x)}{W_{q}^{\prime}(b)}
$$

looks very similar to (1.3). Intuitively, this is due to the fact that the two problems differ only in what happens at the boundary $b$ (reflection versus absorption), which is translated respectively into the boundary conditions $\bar{\Psi}_{q}^{b}(b)=1,\left(V^{b]}\right)^{\prime}(b)=1$ - see Remark 6.2. In fact, this is the heart of the $W, Z$ theory: problems which differ only via their boundary behavior have similar answers - see Section 6 for further examples.

Drawdowns and drawups. Applications require often the study of the running maximum and of the process reflected at its maximum/drawdown

$$
Y_{t}=\bar{X}_{t}-X_{t}, \quad \bar{X}_{t}=\sup _{0 \leq s \leq t} X_{s}
$$

or that of the running infimum and of the process reflected from below/drawup

$$
\underline{Y}_{t}=X_{t}-\underline{X}_{t}, \quad \underline{X}_{t}=\inf _{0 \leq s \leq t} X_{s}
$$

The first passage times of the reflected processes, called drawdown/regret time and drawup time, respectively, are defined for $d>0$ by

$$
\begin{aligned}
\tau_{d} & :=\inf \{t \geq 0: \bar{X}(t)-X(t)>d\}, \\
\underline{\tau}_{d} & :=\inf \{t \geq 0: X(t)-\underline{X}(t)>d\} .
\end{aligned}
$$

Such times turn out to be optimal in several stopping problems, in statistics [121] in mathematical finance/risk theory (in problems involving dividends at a fixed barrier or capital injections) - see for example [22, 41, 50, 95, $99,100,118,141,143,144]$ and in queueing theory (for example when studying idle times until a buffer reaches capacity) - see for example [60, 61].

Capital injections/bail-outs. A second important problem is that of the expected capital injections necessary to maintain a process positive, before reaching an upper barrier; this involves two reflecting boundaries. Since problems with double reflection live on finite intervals, the possibility to solve them by Laplace transforms seems lost at first; however, their solutions are also expressible in terms of the fundamental scale functions $W_{q}, Z_{q}$.

For example, the joint Laplace transform of the total regulation/capital injections into a spectrally negative process (1.7) reflected at $a$ and of the first up-crossing of a level $b$ is ([78], Thm. 2)

$$
\bar{\Psi}_{q, \theta}^{b}\left(x,[a):=\mathbb{E}_{x}^{[a}\left[e^{-q T_{b}^{[a}-\theta L_{T_{b}^{[a}}^{[a}}\right]=\mathbb{E}_{x}^{[a}\left[e^{\left.-\theta L_{T_{b}^{[a}}^{[a} ; T_{b}^{[a}<e_{q}\right]}=\frac{Z_{q}(x-a, \theta)}{Z_{q}(b-a, \theta)},\right.\right.
$$

where $\mathbb{E}_{x}^{[a}$ denotes the expectation for the process reflected at $a, T_{b}^{[a}$ denotes the corresponding hitting time (6.5), and $e_{q}$ denotes an independent exponential random variable of rate $q$. This factorization is essentially a direct consequence of the strong Markov property. In our view, it is maybe the most important first passage law - see Theorem 6.3.

Joint behavior of the process and its drawdown. The third act in the development of risk theory was the consideration of the joint behavior of the process and its historical maxima or minima, or, equivalently, of the process and its drawdowns or drawups. It turns out that this study, just like the previous problems, may be reduced to finding the $W_{q}, Z_{q}$ functions - see for example Theorem 6.13. 
Contents. We start with a brief review of Lévy processes in Section 2. Section 3 presents the function $W_{q}$ which is the pillar of this field, and includes three remarkable results in which it appears. Section 4 introduces the $Z_{q}$ scale function, and Section 5 introduces a two variables extension $Z_{q}(x, \theta)$ of $Z_{q}(x)$.

We turn next to the extensive and expanding body of knowledge concerning spectrally negative Lévy processes. Our $W, Z$ "cookbook" collects a list of some of our favorite recipes. They come from many recent papers, like $[4,7,22,31-33,78,79,81,126]$ and other papers cited below, and we apologize for any omission. Section 6 alone lists ten of the most important first passage laws, dubbed theorems, an eleventh "meta theorem" including the "Poissonian/Parisian version" of most of the first ten theorems is presented in Section 8, and other twelve results spread throughout the paper are called propositions (this partition was adopted for the same reasons we organize files in folders).

Section 7 reviews some $W, Z$ formulas for smooth Gerber-Shiu functions. Here the smooth Gerber-Shiu function $Z_{q}(x, \theta)$ which corresponds to the overshoot penalty $e^{\theta X_{T_{0}}}$ is replaced by a function $G_{w}(x)$ corresponding to an arbitrary penalty function $w\left(X_{T_{0}}\right)$.

Section 8 reviews $W, Z$ formulas for processes with Poisonian/Parisian observations, and for the more general Omega processes. The idea, which emerged naturally in the last decade in the context of financial modeling, is that "transgressing boundaries" may pass unnoticed, with or without purpose, if observations are not continuous. This gives rise to "soft boundaries", in addition to the traditional reflecting and absorbing "hard boundaries" from the physics world; it seems therefore an important development in the theory of Markov processes. This topic is excellently presented in the article [12], but we go beyond that. Quite surprisigly, despite the fact that the methods of proof are different, we have showed in [20] that several of the Parisian formulas coincide with the classic ones, in terms of two new scale functions (which generalize the classic ones). The same phenomenon was observed in [33] for processes with Parisian observations within a finite buffer, below which absolute ruin occurs. It is still not understood why the classic and (buffered) Parisian laws look identical, once the appropriate scale functions have been identified. Let us note that due to its theoretical and applied implications, this topic constitutes an active field of research, with many open problems, some of which are listed below.

To illustrate the potential applicability of $W, Z$ formulas, we have included in Section 9 an important application: the optimization of dividends, under several objectives. We have chosen this application partly since it is a fundamental brick in the budding discipline of risk networks [17, 18, 20]. We also chose this to emphasize that the famous and still not completely understood de Finetti optimization problem [31, 32, 35, 36, 56, 68, 101, 139] is just one of a family of similar optimization problems which can be tackled via the scale function methodology, some of which may be more tractable than the original. Section 10 illustrates the results on examples like (Sect. 10.1) and exponential claims (Sect. 10.3), and Section 10.4 illustrates the numerical optimization of dividends for the Azcue-Muler example [35].

Section 11 reports on recent results on drawdown problems. The motivation is to explore the idea that in risk control (and optimal consumption/harvesting problems) it may be profitable to base decisions both on the position of the underlying process and on its distance from previous suprema. This suggests basing decisions on Azema-Yor/generalized drawdown/trailing stop times, which involve certain admissible functions of the position and supremum. This framework provides a natural unification of drawdown and classic first passage times.

It was discovered in this context that $W, Z$ formulas continue to hold for spectrally negative Markov processes [100]. The only difference is that in equations like (1.3) and (1.4), $W_{q}(x-a), Z_{q}(x-a, \theta)$ must be replaced by functions with one more variable $W_{q}(x, a), Z_{q}(x, a, \theta)$. Unfortunately, the computation of these scale functions is currently understood in only one particular case outside Lévy processes and diffusions: that of OrnsteinUhlenbeck with phase-type jumps, treated in Jacobsen-Jensen [83]. However, we believe that other diffusions with phase-type jumps will be treated in the future via variations of this approach. For that reason, we decided to present the last Section 11 in the context of spectrally negative Markov processes (note though that this is mostly uncharted territory).

The paper ends with a short chronology in Section 12, and a summary of notations and asymptotic formulas in Sections 12, 13, 13.1.

We hope that our compilation may be of help as a quick introduction to more detailed treatments like $[43,63,85,93,94]$ and also as a cookbook for computing quantities of interest in applications like risk theory, 
mathematical finance, inventory and queueing theory, reliability, etc. We will be forced to make appeal to the literature for many proofs, but some of the most useful methods of attack will be included.

\section{A GLIMPSE OF LÉVy PROCESSES}

A Lévy process [43, 94]. $X=X_{t} \in \mathbb{R}, t \geq 0$ may be characterized by its Lévy-Khinchine/Laplace exponent/symbol $\kappa(\theta)$, defined by

$$
\mathbb{E}_{0}\left[e^{\theta X_{t}}\right]=e^{t \kappa(\theta)},
$$

where $\theta \in \mathcal{D} \subset \mathbb{C}$, and $\mathcal{D}$ includes at least the imaginary axis.

Lévy processes and their reflections (drawdowns and drawups) satisfy a duality result ([43], Prop. VI.3, [94], Lem. 3.5):

Lemma 2.1. For each fixed $t>0$, the pairs $\left(\bar{X}_{t}, X_{t}-\bar{X}_{t}\right)$ and $\left(X_{t}-\underline{X}_{t}, \underline{X}_{t}\right)$ have the same distribution under $P_{0}$.

Remark 2.2. This result is behind the well-known duality between queueing and risk theories, which are concerned with reflected and absorbed processes, respectively. For example, applying it when $t \rightarrow \infty$ to the negative of the Cramér-Lundberg process $-X$, when $\kappa^{\prime}\left(0_{+}\right)>0$, yields the well-known identity between the stationary law of the $\mathrm{M} / \mathrm{G} / 1$ workload process and the infimum $\underline{X}_{\infty}$ of the Cramér-Lundberg risk process - see $[13,14]$, and see $[45,124]$ for further applications.

Remark 2.3. The reflected processes of a Lévy process are Markov processes ([43], Prop. VI.1); therefore, nice results on them and first drawdown /drawup passage times are to be expected. is:

Lévy processes satisfy the well-known Wiener Hopf factorization ([43], Prop. VI.5), a short version of which

Lemma 2.4. Let $G_{t}:=\sup \left\{0 \leq s \leq t: X_{s}=\bar{X}_{t}\right\}$ be the last time the process $X$ equals its supremum before or at time $t\left(t-G_{t}\right.$ is therefore the duration of the last drawdown at time $\left.t\right)$. For any independent exponential random variable $e_{q}$ with rate $q>0$, the pairs $\left(\bar{X}\left(e_{q}\right), \bar{G}\left(e_{q}\right)\right)$ and $\left(X\left(e_{q}\right)-\bar{X}\left(e_{q}\right), e_{q}-\bar{G}\left(e_{q}\right)\right)$ are independent under $P_{0}$.

\subsection{The spectrally negative Lévy risk model}

From now on, $X_{t}, t \geq 0$ will denote a spectrally negative Lévy process. It is natural in applications to restrict to the case when the Laplace exponent has a Lévy-Khinchine decomposition of the form

$$
\kappa(\theta)=\frac{\sigma^{2}}{2} \theta^{2}+p \theta+\int_{(0, \infty)}\left[e^{-\theta y}-1+\theta y\right] \Pi(\mathrm{d} y), \theta \geq 0,
$$

with a Lévy measure $\Pi$ of $-X$ satisfying

$$
\int_{(0, \infty)}\left(y \wedge y^{2}\right) \Pi(\mathrm{d} y)<\infty
$$

(and $\Pi(-\infty, 0)=0) .^{2}$ This implies that the growth (or profit) rate satisfies

$$
\mathbb{E}_{0}[X(1)]=p=\kappa^{\prime}\left(0_{+}\right) \neq \infty,
$$

a reasonable assumption in risk theory.

\footnotetext{
${ }^{2}$ Note that even though $X$ has only negative jumps, for convenience we work with the Lévy measure of $-X$.
} 
This assumption excludes Lévy measures like $\Pi(\mathrm{d} x)=x^{-2} \mathrm{~d} x$ and $\alpha$-stable processes with $\alpha \in(0,1)$, but it allows $\alpha$-stable processes with $\alpha \in[1,2)$ (the Lévy measure is allowed to have infinite mean, as long as $\left.\int_{1}^{\infty} y \Pi_{Z}(\mathrm{~d} y)<\infty\right)$.

Remark 2.5. $X_{t}$ is a Markovian process with infinitesimal generator $\mathcal{G}$, which acts on $h \in C_{0}^{2}\left(\mathbb{R}_{+}\right)$by ([137], Thm. 31.5)

$$
\mathcal{G} h(x)=\frac{\sigma^{2}}{2} h^{\prime \prime}(x)+p h^{\prime}(x)+\int_{(0, \infty)}\left[h(x-y)-h(x)+y h^{\prime}(x)\right] \Pi(\mathrm{d} y)
$$

(where we used (2.3)). Incidentally, this may be formally written as $\mathcal{G}=\kappa(D)$, where $D$ denotes the differentiation operator.

If furthermore the jumps of the process have a finite mean $\int_{0}^{\infty} y \Pi(\mathrm{d} y)<\infty$ (but not necessarily finite mass, which allows including interesting examples like the Gamma process [58]), we may rewrite (2.2) as

$$
\kappa(\theta)=\frac{\sigma^{2}}{2} \theta^{2}+c \theta+\int_{(0, \infty)}\left[e^{-\theta y}-1\right] \Pi(\mathrm{d} y), \quad \theta \geq 0, \quad c:=p+\int_{(0, \infty)} z \Pi(\mathrm{d} z),
$$

which reflects a decomposition into a Brownian motion with parameters $(c, \sigma)$ and the negative of a subordinator. We will call this the Brownian perturbed finite mean subordinator risk model.

A further particular case to bear in mind is that when the Lévy measure has finite mass $\Pi(0, \infty)=\lambda<\infty$. We may write then $\Pi(\mathrm{d} z)=\lambda F(\mathrm{~d} z)$, and rewrite the process and its symbol as

$$
X_{t}=x+\sigma B_{t}+c t-\sum_{i=1}^{N_{t}^{(\lambda)}} C_{i}, \quad \kappa(\theta)=\frac{\sigma^{2} \theta^{2}}{2}+c \theta+\lambda \widehat{f}_{C}(\theta)-\lambda,
$$

where $B_{t}$ is the Wiener process, $C_{i}, i=1,2, \ldots$ are i.i.d. nonnegative jumps with distribution $F(\mathrm{~d} z)$, arriving after exponentially distributed times with mean $1 / \lambda$, and $\widehat{f}_{C}$ denotes the Laplace transform of $C_{i}$. This is the Brownian perturbed compound Poisson risk model [57]. If furthermore $X_{t}$ has paths of bounded variation, which happens if and only if $\sigma=0$, we obtain the classic Cramér-Lundberg risk model (1.1). The simplicity of this case comes from the fact that its down-ladder times are discrete, which made it a natural favorite in risk theory.

Finally, let us mention the so-called "Pollaczek-Khinchine" processes which satisfy a generalization of the Pollaczek-Khinchine formula [57]. The most general version due to [77] is obtained by putting together a negative subordinator satisfying $\int_{(0, \infty)}(y \wedge 1) \Pi(\mathrm{d} y)<\infty$ and an independent spectrally negative zero mean perturbation satisfying (2.3). The advantage of this class comes from the fact that its jump down-ladder times are discrete.

State dependent Lévy processes. Nowadays there is also considerable interest in "Lévy processes with state dependent coefficients". For example Albrecher and Cani studied the Cramér-Lundberg process with affine dividends $X_{t}=x+\int_{0}^{t}\left(p-k X_{s}\right) \mathrm{d} s-\sum_{0}^{N_{t}^{(\lambda)}} C_{i}$ [2], and [55] studied a more general "Lévy driven Langevin model" $\mathrm{d} X_{t}=p\left(X_{t}\right) \mathrm{d} t-\mathrm{d} S_{t}$, where $S_{t}$ is a spectrally positive Lévy process.

\section{The SCAle FUnCtion $W_{q}$ And its LOGaRithmic DeRIVAtive $\nu_{q}$}

\subsection{Introduction}

First passage results for spectrally negative Lévy processes are remarkably simpler than in the general case. Here everything reduces finally to the determination of the "scale functions" $W_{q}(x): \mathbb{R}_{+} \rightarrow[0, \infty), q \geq 0$ 
defined on the positive half-line by the Laplace transform (3.1), and extended to be 0 on $\mathbb{R}_{-}$.

$$
\int_{0}^{\infty} \mathrm{e}^{-s x} W_{q}(x) \mathrm{d} x=\frac{1}{\kappa(s)-q}, \quad \forall s>\Phi(q)
$$

where $\Phi_{q}$ is the largest nonnegative root of the Cramér-Lundberg equation

$$
\Phi(q):=\sup \{s \geq 0: \kappa(s)-q=0\}, \quad q \geq 0 .
$$

The scale function $W_{q}(x)$ is continuous and increasing on [0, ) [44] ([43], Thm. VII.8, [94], Thm. 8.1).

Applying optional stopping at $T_{x,+}$ to the Wald martingale $e^{\Phi_{q} X_{t}-q t}$ yields the fundamental identity

$$
\mathbb{E}_{a}\left[e^{-q T_{x,+}}\right]=e^{-(x-a) \Phi_{q}}=P_{a}\left[\bar{X}\left(e_{q}\right)>x\right],
$$

where $e_{q}$ is an independent exponential random variable with parameter $q$ (thus, $T_{x,+}, x \geq 0$ is a subordinator, with Laplace exponent $\Phi_{q}[43]$, Thm. VII.1).

Remark 3.1. In the case of general Lévy processes, solving first passage problems rests on the Wiener-Hopf factorization of the Laplace exponent with killing $\kappa(s)-q$ ([43], Prop. VI.5) (for meromorphic exponents, this means the identification and separation of the positive and negative roots, see Sect. 6.5.4 in [94] for details. ${ }^{3}$ The factorization simplifies considerably for Lévy processes which jump in only one direction (as is the case in queueing and risk theory), since then one part of the factorization involves only the root $\Phi(q)$ defined in (3.2). Typically, this renders the factorization unnecessary, with most things expressable in terms of the pair of functions $\kappa, \Phi$.

For example, in the spectrally negative case, the moment generating function of the drawdown $Y_{e_{q}}$ at an exponential time $e_{q}$, equal to that of $-\underline{X}_{e_{q}}$, satisfies ([94], Thm. 4.8)

$$
\mathbb{E}_{0}\left[e^{-s Y_{e_{q}}}\right]=\frac{s-\Phi(q)}{\kappa(s)-q} \frac{q}{\Phi_{q}} .
$$

When $q \rightarrow 0$, this becomes the Pollaczek-Khinchine formula

$$
\mathbb{E}_{0}\left[e^{-s Y_{\infty}}\right]=\frac{\kappa^{\prime}\left(0_{+}\right) s}{\kappa(s)},
$$

which made some authors call (3.4) the generalized Pollaczek-Khinchine formula.

Another case in which the factorization is easy to compute is that of two-sided phase-type jumps - see for example [15].

The smooth two-sided exit problem. The most fundamental first passage problem is the classic gambler's winning problem [69] ([142], Thm. 3; [42], (6)). This is an extension of (3.3), in which one kills the process upon reaching a lower barrier $a$ which may be taken w.l.o.g. to be 0 .

Proposition 3.2. For any $b>0$ and $x \in[0, b]^{4}$,

$$
\bar{\Psi}_{q}^{b}(x)=\mathbb{E}_{x}\left[e^{-q T_{b,+}} \mathbb{1}_{\left\{T_{b,+}<T_{0}\right\}}\right]=\frac{W_{q}(x)}{W_{q}(b)}:=e^{-\int_{x}^{b} \nu_{q}(s) \mathrm{d} s} .
$$

\footnotetext{
${ }^{3}$ For a proof using the Kella-Whitt martingale, see Theorem 4.8 in [94].

${ }^{4}$ Note that (3.5) may be obtained by stopping the martingale $W_{q}\left(X_{t}\right)$ at $T_{b,+}$.
} 
Analytically, $\nu_{q}(s)$ is the "logarithmic derivative of $W_{q}$ from the right" ([94], (8.26)),

$$
\nu_{q}(s)=\frac{W_{q}^{\prime}(s+)}{W_{q}(s)}
$$

and the "from the right" will be omitted below since we assume $W_{q} \in C^{1}(0, \infty){ }^{5}$

Remark 3.3. Two probabilistic interpretations of $\nu_{q}$. We are trying to avoid as much as possible in our review the use of excursion theory. However, in preparation for the very important problem of dividends paid under a constant barrier policy, we will make an exception, and present a "homemade" version of excursion theory, explained in this remark and in Section 11.

1. It has been noted in [8] that the last equality in (3.5) may be interpreted as the probability that no arrival has occurred between times $x$ and $b$, for a nonhomogeneous Poisson process of rate $\nu_{q}(s)$.

This checks with the probabilistic definition of $\nu_{q}(s)$ provided by excursion theory:

$$
\nu_{q}(x):=n\left[\bar{\epsilon}>x, s(\epsilon) \leq e_{q}\right]
$$

where $n(d \epsilon)$ is the characteristic measure of the Poisson process of downward excursions $\epsilon$ from a running maximum, $\bar{\epsilon}$ denotes the height of a downward excursion, $s(\epsilon)$ denotes the starting time of an excursion, and $e_{q}$ is an independent exponential random variable of rate $q$ - see for example [43], ([62], (12)).

2. We would prefer to avoid excursion theory in our cookbook; however, the concept of excursion is too fundamental to be avoided. We proceed therefore with a "homemade" version of excursion theory for spectrally negative processes, based on excising the negative excursions of $X_{t}$. It has been noted in $[11,30]$ that differentiating the last equality in (3.5) yields

$$
\frac{\mathrm{d}}{\mathrm{d} s} \bar{\Psi}_{q}^{b}(s)-\nu_{q}(s) \bar{\Psi}_{q}^{b}(s)=0, \quad \bar{\Psi}_{q}^{b}(b)=1 .
$$

One may recognize here the Kolmogorov equation for the probability that a deterministic process $\widetilde{X}(s)=s$ starting at 0 , and also killed at rate $\nu_{q}(s)$ either when a negative excursion larger that $s$ occurs, or when an exponential clock of rate $q$ ticks, reaches $b$ before being killed. "It turns out" that $\widetilde{X}(s)$ may be obtained by taking the running maximum value $s$ as time parameter, and by excising the negative excursions of $X(t)$ which are larger than $s$. This interpretation is fundamental, and holds for spectrally negative Markov processes as well - see the last Section 11, in particular Remark 11.1. $\widetilde{X}(s)$ will be called from now on "excised ladder process".

Note that the quotation marks in "it turns out" above and below mean that the statement can be left as an exercise for the Cramér-Lundberg process, but needs in general careful treatment, which is beyond the scope of our cookbook.

Summarizing this discussion, we retain that $\nu_{q}(s)$ represents the rate of the exponentially distributed period of time the process spends at an upward creeping moment (when $\bar{X}_{t}=X_{t}$ ), before a downward excursion bigger than $s$ occurs, and before an exponential clock of rate $q$ ticks [94].

This interpretation of $\nu_{q}(s)$ is especially important in the de Finetti problem (6.1), where we will exploit the fact that the expected dividends $v_{q}(b)$ paid at a fixed barrier $b$ when starting from $b$ equal the expected

\footnotetext{
${ }^{5}$ Since (3.5) is the Laplace transform of the density of $T_{b,+}$, with absorption at $T_{0}$, a Laplace inversion will recover the corresponding density.
} 
discounted time until killing of $\widetilde{X}$. This yields finally the simple relation

$$
v_{q}(b):=\mathbb{E}_{b}\left[\int_{0}^{T_{0,-}} e^{-q t} \mathrm{~d} U_{t}\right]=\nu_{q}(b)^{-1} .
$$

This relation can be extended to spectrally negative Markov processes with generalized drawdown (11.29).

The smoothness of $W_{q}$. Regarding the smoothness of the scale function, it holds that $W_{q} \in C^{1}(0, \infty)$ iff the Lévy measure has no atoms, or $X$ is of unbounded variation. If a Gaussian component is present $(\sigma>0)$, then furthermore $W_{q} \in C^{2}(0, \infty)$. See [52,64] for further results on smoothness, and [101] for the case of completely monotone Lévy measures. ${ }^{6}$ Below, we will always assume that $W_{q}(\cdot)$ is smooth enough to satisfy the equation $\mathcal{G}\left(W_{q}\right)(x)=q W_{q}(x)$ in the classical sense.

The behavior in the neighborhood of zero of $W_{q}$ can be obtained from the behavior of its Laplace transform (3.1) at $\infty$ ([91], Lem. 4.3-4.4; [85], Lem. 3.2-3.3):

$$
\begin{gathered}
W_{q}(0)=\lim _{s \rightarrow \infty} \frac{s}{\kappa(s)-q}= \begin{cases}\frac{1}{c}, & \text { if } X \text { is of bounded variation/Cramér-Lundberg } \\
0, & \text { if } X \text { is of unbounded variation }\end{cases} \\
W_{q}^{\prime}\left(0_{+}\right)=\lim _{s \rightarrow \infty} s\left(\frac{s}{\kappa(s)-q}-W_{q}(0)\right)= \begin{cases}\frac{q+\Pi(0, \infty)}{c^{2}}, & \text { if } X \text { is of bounded variation } \\
\frac{2}{\sigma^{2}}, & \text { if } \sigma>0, \\
\infty, & \text { if } \sigma=0 \text { and } \Pi(0, \infty)=\infty\end{cases}
\end{gathered}
$$

Following the same approach, we may recursively compute $W_{q}^{\prime \prime}(0)$, etc. (these Taylor coefficients may be used in Padé approximations, see [26]). We find, when the jump distribution has a density $f$, that

$$
\begin{aligned}
W_{q}^{\prime \prime}\left(0_{+}\right) & =\lim _{s \rightarrow \infty} s\left(s\left(\frac{s}{\kappa(s)-q}-W_{q}(0)\right)-W_{q}^{\prime}\left(0_{+}\right)\right) \\
& = \begin{cases}\frac{1}{c}\left(\left(\frac{\lambda+q}{c}\right)^{2}-\frac{\lambda}{c} f(0)\right), & \text { if } X \text { is of bounded variation } \\
-c\left(\frac{2}{\sigma^{2}}\right)^{2}, & \text { if } \sigma>0\end{cases}
\end{aligned}
$$

where the notation for the compound Poisson case is as in (1.1). This equation is important in establishing the nonnegativity of the optimal dividends barrier - see Example 10.6.

We offer now as appetizer a strikingly beautiful recent application of the scale function due to (14) in [71] to the calculation of the maximal severity of ruin [123] - see also Prop XII.2.15 in [1] for the compound Poisson case.

Proposition 3.4. Let

$$
\eta:=T_{0}=\inf \left\{t>T_{0,-}: X_{t}=0\right\}
$$

denote the hitting time of 0 ("recovery after ruin") - see also (6.13).

The cumulative distribution function of the maximal severity of ruin $-\underline{X}_{\eta}$ (i.e. the absolute value of the infimum of the process before "recovery after ruin") is given by

$$
P_{x}\left[-\underline{X}_{\eta}<u, T_{0,-}<\infty\right]=\frac{W(x+u)-W(x)}{W(u)} .
$$

\footnotetext{
${ }^{6}$ This paper shows that if the Lévy measure has a completely monotone density, $W_{\Phi_{q}} \in C^{\infty}(0, \infty)$, and $W_{\Phi_{q}}^{\prime}$ is also completely monotone.
} 
Proof. By requiring that the first passage time precedes reaching $-u$ and by using the gambler's winning identity (3.5) one obtains that

$$
P_{x}\left[-\underline{X}_{\eta}<u, T_{0,-}<\infty\right]=\int_{0}^{u} P_{x}\left[-X_{T_{0,-}} \in \mathrm{d} y, T_{0,-}<\infty\right] \frac{W(u-y)}{W(u)} .
$$

On the other hand, by considering the event of reaching 0 , but never reaching $-u$ at all we get

$$
\Psi(x)-\Psi(x+u)=\int_{0}^{u} P_{x}\left[-X_{T_{0,-}} \in \mathrm{d} y, T_{0,-}<\infty\right] \bar{\Psi}(u-y),
$$

and by using (1.5) and (3.12) it follows that

$$
W(x+u)-W(x)=\int_{0}^{u} P_{x}\left[-X_{T_{0,-}} \in \mathrm{d} y, T_{0,-}<\infty\right] W(u-y)=P_{x}\left[-\underline{X}_{\eta}<u, T_{0,-}<\infty\right] W(u) .
$$

Remark 3.5. We end this subsection by noting that showing that the function defined by (1.3) has Laplace transform (3.1) (up to a constant), is not trivial.

The first construction via excursion theory is due to Theorem VII.8 in [43]. Other elegant solutions are due to [119], who used a Kennedy type martingale, and to (3) in [126], who constructed the scale function as

$$
W_{q}(x)=\Phi_{q}^{\prime} e^{\Phi_{q} x}-u_{q}(-x)=\Phi_{q}^{\prime}\left(e^{x \Phi_{q}}-\mathbb{P}_{x}\left[T_{\{0\}}<e_{q}\right]\right), x \geq 0
$$

where $u_{q}$ is the potential density - see (6.14) below for a proof of the last formula, which can be easily implemented via Monte Carlo simulation. ${ }^{7,8}$

The simplest solution maybe is to reduce to the case $q=0$ by using the easily checked Esscher transform relation

$$
W_{q}(x)=e^{x \Phi_{q}} W_{0}^{\left(\Phi_{q}\right)}(x)
$$

Here $W_{0}^{\left(\Phi_{q}\right)}(x)$ denotes the 0-scale function with respect to the "Esscher transformed" measure $P^{\left(\Phi_{q}\right)}$ (in general, the transform $P^{(r)}$ of the measure $P$ of a Lévy process with Laplace exponent $\kappa(s)$ is the measure of the Lévy process with Laplace exponent $\kappa(s+r)-\kappa(r)$, with $r$ in the domain of $\kappa(\cdot)$ ([1], Prop. 4.2; [94], 3.3 p. 83).

The advantage of $W_{0}^{\left(\Phi_{q}\right)}(x)$ is that this is a monotone bounded function, with values in the interval $\left(\lim _{s \rightarrow \infty} \frac{s}{\kappa(s)}, \frac{1}{\kappa^{\prime}\left(\Phi_{q}\right)}\right)$. Therefore, for numerical computation of $W_{q}$ it will be useful to replace it by $W_{0}^{\left(\Phi_{q}\right)}(x)$, with Laplace transform

$$
\widehat{W}^{\left(\Phi_{q}\right)}(s)=\frac{1}{\kappa\left(s+\Phi_{q}\right)-q}=\frac{1}{\kappa\left(s+\Phi_{q}\right)-\kappa\left(\Phi_{q}\right)}:=\frac{1}{\kappa^{\left(\Phi_{q}\right)}(s)},
$$

(removing thus the exponential growth). Padé and Laguerre approximations of (3.14) are provided in [29].

Another probabilistic interpretation of (3.14) is

$$
W_{q}(x)=e^{x \Phi_{q}} \widetilde{L}_{q}(x),
$$

${ }^{7}(3.13)$ holds trivially for $x \in \mathbb{R}_{-}$as well, when it reduces to $\mathbb{P}_{x}\left[T_{\{0\}}<e_{q}\right]=e^{x \Phi_{q}}$, which may be interpreted as the value of a payment of 1 at the hitting time $T_{\{0\}}$.

${ }^{8}$ Noting finally that $u_{q}(x), x \in \mathbb{R}_{+}$is exponential given by $u_{q}(x)=\Phi_{q}^{\prime} e^{-\Phi_{q} x}, x \geq 0$ and letting $u_{q}^{+}(x)=\Phi_{q}^{\prime} e^{-\Phi_{q} x}, x \in \mathbb{R}$ denote the analytic continuation of $u_{q}(x), x \geq 0$ yields yet another representation $W_{q}(x)=u_{q}^{+}(-x)-u_{q}(-x)[21]$. 
where $\widetilde{L}_{q}(b)=E\left[\int_{0}^{T_{b,+}} e^{-q t} \mathrm{~d} L_{t}^{0}\right]=L_{T_{b,+} \wedge e_{q}}^{0}=\Phi_{q}^{\prime}-e^{-\Phi_{q} x} u_{q}(-x)$ is the expected discounted occupation time at 0 , starting at 0 , before up-crossing the level $b([43], \mathrm{V}(18))$. This relation extends to the spectrally negative Markov additive processes (SNMAP) context ([78], (2),(12)) and has been used for computing numerically the SNMAP matrix scale function [80].

Remark 3.6. $\Phi_{q}$ and the other roots of the Cramér-Lundberg equation $\kappa(s)-q=0$ play a central role in asymptotics computations. Clearly, $\Phi_{q}$ is the asymptotically dominant singularity

$$
W_{q}(x) \sim \Phi_{q}^{\prime} e^{x \Phi_{q}}=\frac{e^{x \Phi_{q}}}{\kappa^{\prime}\left(\Phi_{q}\right)}, \quad x \rightarrow \infty
$$

The other poles of the right hand side of (3.1) (the roots of the Cramér-Lundberg equation) intervene, when they exist, in the asymptotics of the eventual ruin probabilities when $\kappa^{\prime}\left(0_{+}\right)>0$ and in their numerical approximations - see for example [9, 23-26].

\subsection{Two resolvents in terms of the $W_{q}(x)$ function}

We will recall here two fundamental resolvent formulas expressed in terms of $W_{q}$. Resolvents are at a level of sophistication above the other concepts reviewed in this paper, and these results will not be proved. However, once accepted, they provide us with a convenient point of entrance in our topic.

We introduce first a notation style used throughout the paper.

Remark 3.7. Our cookbook will require notations for several types of boundaries for example absorbing, reflecting, refracting, and Parisian/Poisonian stopping or reflecting. To deal with these five cases, it is convenient, following [81], to append the state space to the specification of a process; the five cases above will be denoted

below by $b \mid, b], b[, b \vdots, b\}$ for an upper boundary, and for a lower boundary by $\mid a,[a] a,, \vdots a,\{a$. For drawdown boundaries, the respective notations will be $\bar{d}, \widehat{d}, \breve{d}, \ddot{d}, \widetilde{d}$. Note that the term "boundary" for the refracting and Parisian cases is meant in the sense of a discontinuous "regime switching" in the drift and killing parameters of the process, respectively. This convention gives suggestive notations when composing several mechanisms. For example, for the "classic reflection above at $b$, with Parisian reflection below at $b_{0}$ and absolute ruin at $a<b_{0}$ " studied in $[33,130]$, the notation for the corresponding state space would be $\mid a,\left\{b_{0}, b_{1}\right]$. Such boundaries are useful in optimal control [132].

Note that absorption delimiters like $\mid a$ and $b \mid$ and may and will be often omitted without confusion (so the default for an unspecified end-point is absorbing).

Proposition 3.8. Put $W_{q}(x, a)=W_{q}(x-a)$ (as a reminder that these formulas hold also for spaceinhomogeneous models, like for example for refracted processes [114]). ${ }^{9}$

A) For any bounded interval $[a, b]$ and any Borel set $B \subset[a, b]$, let

$$
U_{q}^{|a, b|}(x, B)=\mathbb{E}_{x}\left[\int_{0}^{T_{a,-} \wedge T_{b,+}} e^{-q t} \mathbb{1}_{\left\{X_{t} \in B\right\}} \mathrm{d} t\right],
$$

denote the q-resolvent of the spectrally negative Lévy process killed outside the interval [a,b]. Then ([142]; [42], Thm. 1; [94], Thm. 8.7; [81], (14); [104], Thm. 2.2; [114], Thm. 1) $U_{q}^{|a, b|}(x, B)=\int_{a}^{b} \mathbb{1}_{\{y \in B\}} u_{q}^{|a, b|}(x, y) \mathrm{d} y$, with resolvent density

$$
u_{q}^{|a, b|}(x, y)=\frac{P_{x}\left[X_{e_{q}} \in d y\right]}{q d y}=\frac{W_{q}(x, a)}{W_{q}(b, a)} W_{q}(b, y)-W_{q}(x, y) .
$$

\footnotetext{
${ }^{9}$ One of the nice things about the toolkit is that switching to inhomogeneous skip-free processes just requires changing $x-a$ to $x, a$. The only thing specific to Lévy (and refracted) setting is that $W$ is quasi-explicit.
} 
Note also the following identities in limiting cases - see for example Chapter 8.4 in [94]:

$$
\begin{aligned}
(q \mathrm{~d} y)^{-1} P\left(X_{e_{q}} \in \mathrm{d} y\right) & =\Phi^{\prime}(q) e^{-\Phi(q) y}-W_{q}(-y), \\
(q \mathrm{~d} y)^{-1} P\left(X_{e_{q}} \in \mathrm{d} y, e_{q}<T_{b,+}\right) & =e^{-\Phi(q) b} W_{q}(b-y)-W_{q}(-y), \\
(q \mathrm{~d} y)^{-1} P_{b}\left(X_{e_{q}} \in \mathrm{d} y, e_{q}<T_{0}\right) & =e^{-\Phi(q) y} W_{q}(b)-W_{q}(b-y),
\end{aligned}
$$

where $b>0$ and the killing rate $q \geq 0$ is implicit.

B) The q-resolvent of a spectrally negative Lévy process absorbed below at a and reflected above at $b$ (see (1.7) for definition of reflection) has the resolvent density ([81], (21); [104], Thm. 2.4)

$$
u_{q}^{\mid a, b]}(x, y)=\frac{W_{q}(x, a)}{W_{q}^{\prime}(b, a)}\left(W_{q}^{\prime}(b, y)+W_{q}(0) \delta_{b}(\mathrm{~d} y)\right)-W_{q}(x, y)
$$

where the derivative is taken with respect to the first variable.

Remark 3.9. Letting $b \rightarrow \infty$ in (3.16) we find the resolvent on intervals bounded only below for any Borel set $B \subset[a, \infty)$, which is closely related to Dickson's formula in the actuarial literature

$$
U_{q}^{\mid a}(x, B)=\int_{a}^{\infty} \mathbb{1}_{\{y \in B\}} u_{q}^{\mid a}(x, y) \mathrm{d} y, \quad u_{q}^{\mid a}(x, y)=W_{q}(x-a) e^{-\Phi(q)(y-a)}-W_{q}(x-y) .
$$

Remark 3.10. For other resolvent laws involving all possible combinations of boundary conditions (reflection or/and absorption), see [81, 94, 104]. Note that the proofs use typically excursion theory. One exception is Theorem 4.1 in [132], who compute the resolvent density $u_{q, \lambda}^{]^{0}}(x, y)$ with Parisian reflection at Poisson observation times of intensity $\lambda$. The proof uses the Markov property in the bounded variation case, and a Laplace transform approach in the unbounded variation case.

\section{Obtaining the $Z_{q}(x)$ FUnCtion In teRms of $W_{q}(x)$ By USing THE RESOLVENT}

The first resolvent formula will now be used to introduce the second pillar of this theory, the scale function $Z_{q}$, which intervenes in the "non-smooth-exit law" below. Using this together with the "smooth-exit law" (3.5) will be essential in deriving the other recipes offered below.

Proposition 4.1. A) The Laplace transform of the time until the lower boundary 0 , if this precedes an upper boundary $b>0$, is given by ([22], (10))

$$
\Psi_{q}^{b}(x):=\mathbb{E}_{x}\left[e^{-q T_{0}} ; T_{0}<T_{b,+}\right]=Z_{q}(x)-\frac{W_{q}(x)}{W_{q}(b)} Z_{q}(b),
$$

where $Z_{q}(x)=1+q \bar{W}_{q}(x), \bar{W}_{q}(x)=\int_{0}^{x} W_{q}(u) \mathrm{d} u$.

B) The Laplace transform of the time until the lower boundary 0 in the presence of reflection at an upper boundary $b \geq 0$ is ([32], Prop. 5.5;[78], Thm. 6)

$$
\Psi_{q}^{b]}(x):=\mathbb{E}_{x}^{b]}\left[e^{-q T_{0}^{b]}}\right]=Z_{q}(x)-\frac{W_{q}(x)}{W_{q}^{\prime}(b)} Z_{q}^{\prime}(b),
$$


where $\mathbb{E}^{b]}$ denotes expectation for the process reflected from above at $b$ and

$$
T_{0}^{b]}=T_{0} \mathbb{1}_{\left\{T_{0}<T_{b,+}\right\}}+\tau_{b} \mathbb{1}_{\left\{T_{b,+}<T_{0}\right\}}
$$

denotes the first passage below 0 under this measure (recall that $\tau_{b}$ is a drawdown time (1.10), or, equivalently, the time when the process starting at $b$ and Skorokohod reflected at $b$ is ruined ${ }^{10}$ ).

Here is a proof of Proposition 4.1, borrowed from [114] (who consider the more general case of Omega models).

Proof. A) Put $T=\min \left(T_{0}, T_{b,+}\right)$, and consider the elementary identity:

$$
\int_{0}^{T} q e^{-q t} \mathrm{~d} t=1-e^{-q T}
$$

By denoting

$$
\bar{W}_{q}(x):=\int_{0}^{x} W_{q}(y) \mathrm{d} y,
$$

taking expectation and using the resolvent formula (3.16), we get

$$
\begin{gathered}
q \int_{0}^{b} u_{q}^{|0, b|}(x, y) \mathrm{d} y=1-\frac{W_{q}(x)}{W_{q}(b)}-\Psi_{q}^{b}(x) \Leftrightarrow \\
q\left(\frac{W_{q}(x)}{W_{q}(b)} \int_{0}^{b} W_{q}(b-y) \mathrm{d} y-\int_{0}^{x} W_{q}(x-y) \mathrm{d} y\right)=1-\frac{W_{q}(x)}{W_{q}(b)}-\Psi_{q}^{b}(x) \Longrightarrow \\
\Psi_{q}^{b}(x)=1-\frac{W_{q}(x)}{W_{q}(b)}-q\left(\frac{W_{q}(x)}{W_{q}(b)} \bar{W}_{q}(b)-\bar{W}_{q}(x)\right)=1+q \bar{W}_{q}(x)-\frac{W_{q}(x)}{W_{q}(b)}\left(1+q \bar{W}_{q}(b)\right) .
\end{gathered}
$$

Putting now $Z_{q}(x)=1+q \bar{W}_{q}(x)$ yields the result.

B) Applying the same steps to $T_{0}^{b]}$, we find

$$
\begin{aligned}
\mathbb{E}_{x}\left[\int_{0}^{T_{0}^{b]}} q e^{-q t} \mathrm{~d} t\right]=\mathbb{E}_{x}\left[1-e^{-q T_{0}^{b]}}\right]=1-\Psi_{q}^{b]}(x) & = \\
q\left(\frac{W_{q}(x)}{W_{q}^{\prime}(b)}\left(\int_{0}^{b} W_{q}^{\prime}(b-y) \mathrm{d} y+W_{q}(0)\right)-\int_{0}^{b} W_{q}(x-y) \mathrm{d} y\right) & \Longrightarrow \\
\Psi_{q}^{b]}(x)=1-q\left(\frac{W_{q}(x)}{W_{q}^{\prime}(b)} W_{q}(b)-\bar{W}_{q}(x)\right) & =Z_{q}(x)-\frac{W_{q}(x)}{W_{q}^{\prime}(b)} Z_{q}^{\prime}(b) .
\end{aligned}
$$

Remark 4.2. These two proofs illustrate the very important method of integrating resolvent densities - see [81] for a compendium of resolvent formulas. For a direct proof not using resolvents, in the case of Brownian motion, see Theorem 1.1 in [116].

Remark 4.3. Note the similar structure of (4.1) and (4.2) (a phenomenon which will keep recurring below). Formally, switching from absorption at $b$ to the measure $\mathbb{E}^{b]}$ involving reflection at $b$ only requires switching the respective boundary conditions $\Psi_{q}^{b}(b)=0,\left(\Psi_{q}^{b]}\right)^{\prime}(b)=0$. Now the first boundary condition is obvious, like any absorption boundary condition, but not the second.

\footnotetext{
${ }^{10}$ When $x=b,(4.3)$ simplifies to $T_{0}^{b]}=\tau_{b}$.
} 
Let us examine now a "failed direct approach" to establish

$$
\left(\Psi_{q}^{b]}\right)^{\prime}(b)=0 \Leftrightarrow \Psi_{q}^{b]}(b-\epsilon)-\Psi_{q}^{b]}(b)=o(\epsilon) .
$$

Using now the decomposition (4.3) yields

$$
\begin{aligned}
& \Psi_{q}^{b]}(b)-\Psi_{q}^{b]}(b-\epsilon)=\Psi_{q}^{b]}(b)-\left(Z_{q}(b-\epsilon)-\frac{W_{q}(b-\epsilon)}{W_{q}(b)} Z_{q}(b)+\frac{W_{q}(b-\epsilon)}{W_{q}(b)} \Psi_{q}^{b]}(b)\right) \\
& =\Psi_{q}^{b]}(b)\left(1-\frac{W_{q}(b-\epsilon)}{W_{q}(b)}\right)-\left(Z_{q}(b-\epsilon)-\frac{W_{q}(b-\epsilon)}{W_{q}(b)} Z_{q}(b)\right) \\
& =\epsilon\left[\Psi_{q}^{b]}(b) \frac{W_{q}^{\prime}(b)}{W_{q}(b)}+\left(Z_{q}^{\prime}(b)-\frac{W_{q}^{\prime}(b)}{W_{q}(b)} Z_{q}(b)\right)\right]+o(\epsilon)=\epsilon\left[Z_{q}^{\prime}(b)+\frac{W_{q}^{\prime}(b)}{W_{q}(b)}\left(\Psi_{q}^{b]}(b)-Z_{q}(b)\right)\right]+o(\epsilon) .
\end{aligned}
$$

The boundary condition on the derivative is equivalent thus to the boundary condition on the function $\Psi_{q}^{b]}(b)=$ $\mathbb{E}_{b}^{b]}\left[e^{-q T_{0}^{b]}}\right]=Z_{q}(b)-\frac{W_{q}(b)}{W_{q}^{\prime}(b)} Z_{q}^{\prime}(b)$, which we wanted to avoid establishing. A more sophisticated approach is thus needed. For the Cramér-Lundberg model, the boundary condition (4.6) on the derivative has been established in [111], using the regenerative property of the Poisson process at claim instants (their proof is quite ingenious). For spectrally negative Lévy processes, the use of excursion theory seems unavoidable.

Remark 4.4. The Propositions 3.2-4.1 and most of the results in this review may be modified to apply formally to the context of spectrally negative and spectrally positive Markov processes, which include for example the continuous state-space branching processes (CSBP) - see for example Chapter 12 in [94] (in particular Thm. 12.8), and the continuous-state branching processes with immigration (CBI) introduced by Kawazu and Watanabe [92], which may characterized in terms of two Laplace exponents $\psi, \kappa$ of spectrally positive Lévy processes. However, while $W, Z$ exist (as functions of two variables), no straightforward method for their computation is available. ${ }^{11}$

Remark 4.5. Adding (3.5) and (4.1), we find that for $T=\min \left(T_{0}, T_{b,+}\right)$

$$
\mathbb{E}_{x}\left[e^{-q T}\right]=P_{x}\left[T \leq e_{q}\right]=Z_{q}(x)-\frac{W_{q}(x)}{W_{q}(b)}\left(Z_{q}(b)-1\right)=1-q\left(\frac{W_{q}(x)}{W_{q}(b)} \bar{W}_{q}(b)-\bar{W}_{q}(x)\right),
$$

which recovers ([42], Cor. 1) (up to the omission of $q$ there). Since this must be less than 1, it follows that the function $\frac{\bar{W}_{q}(x)}{W_{q}(x)}$ is increasing, or, equivalently, that $\bar{W}_{q}(x)$ is log-concave, and

$$
\frac{W_{q}^{\prime}(x) \bar{W}_{q}(x)}{W_{q}^{2}(x)}<1
$$

see also [105].

\footnotetext{
${ }^{11}$ Recall that CSBPs are characterized by generators of the form $x \psi(D)$, where $\psi(D)$ is the generator of a spectrally positive Lévy process, and that they may be obtained from spectrally positive Lévy process by a time-change called the Lamperti transformation - see [54]. This acts on the Skorokhod space $\mathbb{D}$ of càdlàg trajectories with values in $E=[0, \infty]$, as follows: for any $f \in \mathbb{D}$, introduce the additive functional $I$ and its inverse $\overleftarrow{I}$, given by $I_{t}=I_{t}(f):=\int_{0}^{t} f(s) \mathrm{d} s \in[0, \infty], \quad \overleftarrow{I}_{t}=\overleftarrow{I}_{t}(g):=\inf \left\{s \geq 0: I_{s}(g)>t\right\}=$ $I_{t}\left(\frac{1}{g}\right) \in[0, \infty]$. The Lamperti transformation $L: \mathbb{D} \rightarrow \mathbb{D}$ is defined by $L(f)=f \circ \overleftarrow{I}$ (note that $L(f)(t)=f(\infty)$ if $\overleftarrow{F}_{t}=\infty$, so that $0, \infty$ indeed are absorbing for $L(f))$. It may be checked that $L$ is a bijection of $\mathbb{D}$, with inverse given by $L^{-1}(g)=g \circ I(g)$. An extension to the CBI case is offered in [51]. However, the Lamperti transformation seems too complicated to yield a method for the computation of $W, Z$ in terms of the Lévy Laplace exponents. It is intriguing to investigate whether simple formulas for $W, Z$ are available in these cases at all.
} 
For a second probabilistic proof of (4.8), consider the time from $b$ to 0 of a reflected process (4.2), which is equal in law to the drawdown time $\tau_{b}{ }^{12}$ Choosing $x=b$ in (4.2) yields

$$
\delta_{q}(b):=\Psi_{q}^{b]}(b)=\mathbb{E}_{0}\left[e^{-q \tau_{b}}\right]=Z_{q}(b)-\frac{W_{q}(b) Z_{q}^{\prime}(b)}{W_{q}^{\prime}(b)}=1-q\left(\frac{W_{q}^{2}(b)}{W_{q}^{\prime}(b)}-\bar{W}_{q}(b)\right) .
$$

Since this must be less than 1, the nonnegativity of the term in parenthesis follows.

Reduction of first passage problems to the computation of the solutions $W_{q}$ and $Z_{q}$ of TSE. It turns out that the solutions of a great variety of first passage problems reduce ultimately to the solutions of the two-sided smooth and non-smooth first passage problems of exit from a bounded interval (TSE). Thus, they may be expressed in terms of $W_{q}$ [42], and further simplified by the introduction of the second scale function $Z_{q}[22]$. Many calculations and inversions of Laplace transforms may be replaced for spectrally negative Lévy processes by the computation of the $W$ and $Z$ scale functions - see [31, 78, 125-127], to cite only a few papers. Furthermore, the formulas reviewed hold as well for spectrally negative Markov additive processes, where the appropriate matrix scale functions were identified in [78, 79, 89], for random walks (the compound binomial risk model) [19], and for positive self similar Markov processes with one-sided jumps [145, 147].

Somewhat surprisingly, it appeared recently that the recipes reviewed below apply equally to spectrally negative Lévy processes with (exponential) Parisian absorption or reflection below [5, 12, 33, 40, 107], with the appropriate scale functions $W, Z$ identified in [20,33]. This mystery was explained in $[104,114,146]$, who showed that the $W, Z$ recipes appropriately extended apply to the general class of Omega models, of which Parisian Poissonian models are a particular case. In fact, the second paper considers even more general models with refraction $[86,90]$.

\section{The three variables $Z_{q}(x, \theta)$ SCAle Function/Dickson-HipP OPERATOR APPLIED TO $W_{q}(\cdot)$}

Let $\widehat{ }_{x} W_{q}(\theta)$ denote the Laplace transform of the shifted scale function ${ }_{x} W_{q}(y):=W_{q}(x+y)$ (the composition of shift with Laplace transform is also called Dickson-Hipp operator).

When the Laplace transform $\mathbb{E}_{x}\left[e^{\theta X_{T_{0}}}\right]$ of the first position of the process after exiting $[0, \infty)$ is of interest, one ends up working with the two variables $Z_{q}$ scale function [22, 78] ([32], Cor. 5.9), defined for $\theta \in \mathbb{C}$ such that the real part $\Re(\theta)>\Phi(q)$ (to ensure integrability) by:

$$
\begin{aligned}
Z_{q}(x, \theta) & =(\kappa(\theta)-q) \int_{0}^{\infty} e^{-\theta y} W_{q}(x+y) \mathrm{d} y:=(\kappa(\theta)-q)_{x} \widehat{x}_{q}(\theta) \\
& =\frac{\kappa(\theta)-q}{\theta-\Phi_{q}} W_{q}(x)+\mathbb{E}_{x}\left[e^{-q T_{0}+\theta X_{T_{0}}} \mathbb{1}_{\left\{T_{0}<\infty\right\}}\right] \\
& =\frac{\kappa(\theta)-q}{\theta-\Phi_{q}} W_{q}(x)+\Psi_{q, \theta}(x), \quad \Re(\theta)>\Phi(q) .
\end{aligned}
$$

(see Cor. 6.6 A for the proof of the last decomposition.) Thus, up to a constant, $Z_{q}(x, \theta)$ is the Laplace transform ${ }_{{ }_{x} W_{q}}(\theta)$ of the shifted scale function ${ }_{x} W_{q}(y):=W_{q}(x+y)$, and the normalization ensures that $Z_{q}(0, \theta)=1$.

Remark 5.1. The first term in the decomposition above is asymptotically dominant for $q>0$. The second term simplifies in the Cramér-Lundberg case when $x=q=0$ to

$$
\mathbb{E}_{0}\left[e^{\theta X_{T_{0}}} \mathbb{1}_{\left\{T_{0}<\infty\right\}}\right]=1-\frac{\kappa(\theta)}{c \theta}=\frac{\widehat{\bar{\Pi}}(\theta)}{c}, \forall \theta>0,
$$

\footnotetext{
${ }^{12}$ That is easily understood by fixing the maximum at $b$, which changes the negative of the drawdown into the Skorokhod reflected process.
} 
identifying the well-known Laplace transform of the deficit at ruin starting from 0 for the Cramér-Lundberg process, where $\widehat{\bar{\Pi}}$ denotes the Laplace transform of the tail of the Lévy measure $\bar{\Pi}(y)=\Pi(y, \infty)$.

The analytic continuation of (5.1) is

$$
Z_{q}(x, \theta)=e^{\theta x}+(q-\kappa(\theta)) \int_{0}^{x} e^{\theta(x-y)} W_{q}(y) \mathrm{d} y, \theta \in \mathbb{C} .
$$

This implies that

$$
\left\{\begin{array}{ll}
Z_{q}(x, \theta)=e^{\theta x}, & x \leq 0 \\
Z_{q}\left(x, \Phi_{q}\right)=e^{x \Phi_{q}}, & x \in \mathbb{R}
\end{array} .\right.
$$

Remark 5.2. We can also identify $Z_{q}(x, \theta)$ via its Laplace transform in $x$ :

$$
\begin{aligned}
& \widehat{Z_{q}}(s, \theta)=(s-\theta)^{-1}\left(\kappa_{q}(\theta)^{-1}-\kappa_{q}(s)^{-1}\right) \kappa_{q}(\theta)=\frac{\kappa(s)-\kappa(\theta)}{s-\theta} \frac{1}{\kappa(s)-q}, \kappa_{q}(s):=\kappa(s)-q \\
& \Longrightarrow \widehat{Z}_{q}(s)=s^{-1} \kappa(s) \kappa_{q}(s)^{-1} .
\end{aligned}
$$

We list now some useful easy to check formulas involving $Z_{q}(x), Z_{q}(x, \theta)$ :

$$
\begin{aligned}
Z_{q}(x) & =1+q \bar{W}_{q}(x)=c W_{q}(x)+\frac{\sigma^{2}}{2} W_{q}^{\prime}(x)-\int_{0}^{x} W_{q}(x-y) \bar{\Pi}(y) \mathrm{d} y \\
\bar{Z}_{q}(x) & :=\int_{0}^{x} Z_{q}(z) \mathrm{d} z=x+q \int_{0}^{x} \int_{0}^{z} W_{q}(w) \mathrm{d} w \mathrm{~d} z \\
Z_{q}^{(1)}(x) & ={\frac{\partial Z_{q}(x, \theta)}{\partial \theta}}_{\theta=0}=\bar{Z}_{q}(x)-\kappa^{\prime}\left(0_{+}\right) \bar{W}_{q}(x), \\
Z_{q}^{\prime}(x, \theta) & =\theta Z_{q}(x, \theta)+(q-\kappa(\theta)) W_{q}(x),
\end{aligned}
$$

where' denotes here and below derivative with respect to $x$ and $\bar{\Pi}(y)=\Pi(y, \infty)$. The second formula for $Z_{q}(x)$ is a particular case of (7.4). Let us check it now when $\sigma>0$ :

$$
\begin{aligned}
1+q \bar{W}_{q}(x) & =1+\int_{0}^{x} \mathcal{G}\left(W_{q}\right)(y) \mathrm{d} y \\
& =1+\frac{\sigma^{2}}{2}\left(W_{q}^{\prime}(x)-W_{q}^{\prime}\left(0_{+}\right)\right)+c\left(W_{q}(x)-W_{q}\left(0_{+}\right)\right)+\int_{0}^{\infty}\left(\int_{0}^{x} W_{q}(y-z) \mathrm{d} y-\int_{0}^{x} W_{q}(y) \mathrm{d} y\right) \Pi(\mathrm{d} z) \\
& =1+\frac{\sigma^{2}}{2}\left(W_{q}^{\prime}(x)-W_{q}^{\prime}\left(0_{+}\right)\right)+c W_{q}(x)+\int_{0}^{\infty}\left(-\int_{x-z}^{x} W_{q}(y) \mathrm{d} y\right) \Pi(\mathrm{d} z)-\mathbb{1}_{\left\{\int \Pi(\mathrm{d} z)<\infty \& \sigma=0\right\}} \\
& =\frac{\sigma^{2}}{2}\left(W_{q}^{\prime}(x)-W_{q}^{\prime}\left(0_{+}\right)\right)+c W_{q}(x)-\int_{0}^{x} W_{q}(x-y) \bar{\Pi}(y) \mathrm{d} y+\mathbb{1}_{\{\sigma>0\}} \\
& =c W_{q}(x)+\frac{\sigma^{2}}{2} W_{q}^{\prime}(x)-\int_{0}^{x} W_{q}(x-y) \bar{\Pi}(y) \mathrm{d} y
\end{aligned}
$$

where we integrated $q W_{q}(x)=\mathcal{G}\left(W_{q}\right)(x)$ with $\mathcal{G}$ given in (2.4), and used Fubini, integration by parts, and $(3.9)$. 
Remark 5.3. For Brownian motion, (5.4) yields

$$
Z_{q}(x)=c W_{q}(x)+\frac{\sigma^{2}}{2} W_{q}^{\prime}(x)=c W_{q}(x)+\frac{W_{q}^{\prime}(x)}{W_{q}^{\prime}(0)} .
$$

Remark 5.4. Note that for $x \leq 0$, it holds that $\bar{W}_{q}(x)=0, Z_{q}(x)=1, \bar{Z}_{q}(x)=x$, and that $Z_{q}(x, \theta)$ is proportional to an Esscher transform; indeed, it is easy to check that $W_{q-\kappa(\theta)}^{(\theta)}(x)=e^{-\theta x} W_{q}(x), Z_{q-\kappa(\theta)}^{(\theta)}(x)=$ $e^{-\theta x} Z_{q}(x, \theta)$. Recall that the Esscher transform refers to an exponential change of measure using the martingale $e^{\theta X_{t}-\kappa(\theta) t}, t \geq 0$. For each $\theta$ in the domain of $\kappa(\cdot)$, the process $X$ remains in the class of spectrally negative Lévy processes, is characterized by the Laplace exponent $\kappa(\cdot+\theta)-\kappa(\theta)$, and $W_{q}^{(\theta)}, Z_{q}^{(\theta)}$ denote the scale functions of $X$ under this change of measure. ${ }^{13}$

Remark 5.5. It is easy to check by taking Laplace transform $[108,125]$ that the convolution of two $W$ scale functions satisfies the equation

$$
W_{q} * W_{\lambda}(x)=\frac{W_{q}(x)-W_{\lambda}(x)}{q-\lambda} .
$$

The analogue formula for the convolution of two $Z$ scale functions is more complicated. When $\sigma=0$, it holds that

$$
\left(Z_{q} * Z_{\lambda}\right)(x)=\left(\frac{Z_{\lambda}-Z_{\xi}}{q-\lambda}\right) *(\bar{\Pi})(x) .
$$

However, the convolution of $W$ and $Z$ is again simple ([12], Lem. 4.1)

$$
W_{q} * Z_{\lambda}(x, \theta)=\frac{Z_{q}(x, \theta)-Z_{\lambda}(x, \theta)}{q-\lambda} .
$$

The history of $\mathbf{Z}$. The second scale function $Z_{q}(x)$ was introduced in the thesis of M. Pistorius (which the first author codirected with A. Kyprianou), as a means of expressing in a simpler way both the results of [42, 142] and some new results involving reflected processes and drawdown stopping (used "Russian options"). See (6) in [22] for the first published reference. Its importance became clearer after its further use in [62, 88, 119, 125-127].

By some historical error, all these papers, as well as the textbook [94], omitted the information that the "birth certificate" of the function $Z$ was signed in the thesis of Pistorius and in [22]. Instead, reference was made to the pioneering work [42], which however contains no $Z$ function.

The three variables extension $Z_{q}(x, \theta)$ was introduced essentially in [22] as an Esscher transform of $Z_{q}(x)-$ see Remark 5.4. Then, the simultaneous papers [78] and Corollary 5.9 in [32] (first submitted in 2011, ArXiv 1110.4965) proposed the direct definition (5.2), without the Esscher transform from previous papers.

Subsequently, $Z_{q}(x, \theta)$ was shown in Theorem 5.3, Corollary 5.9 in [32] to be a particular case of a "smooth Gerber-Shiu function" ([32], Def. 5.2) associated to an exponential payoff $e^{\theta x}$. More precisely, $Z_{q}(x, \theta)$ is the unique "smooth" solution of

$$
\left\{\begin{array}{ll}
(\mathcal{G}-q I) Z_{q}(x, \theta)=0, & x \geq 0 \\
Z(x, \theta)=e^{\theta x}, & x \leq 0
\end{array},\right.
$$

where $\mathcal{G}$ is the Markovian generator (2.4) of the process $X_{t}-$ see (1.12), (5.23), Section 5 in [32] and Section 7.

\footnotetext{
${ }^{13}$ Before the introduction of the notation $Z_{q}(x, \theta)$ in $[32,78]$, results were expressed in terms of Esscher transformed scale functions.
} 
$Z_{q}(x, \theta)$ was used first as generating function for the smooth Gerber-Shiu functions associated to power rewards $1, x, x^{2}$, which were denoted respectively by $Z_{q}, Z_{q}^{(1)}, Z_{q}^{(2)}, \ldots$ Subsequently, it started being used intensively in exponential Parisian ruin problems following the work of [12].

As of recently, several papers $[4,12,20,31,33,78,79,81,86]$ showed that Lévy formulas expressed in terms of $W_{q}(x)$ and $Z_{q}(x)$ or $Z_{q}(x, \theta)$ hold also for doubly reflected processes, ${ }^{14}$ refracted processes, spectrally negative Markov additive processes, processes with Parisian absorption or reflection, and combinations of these features. More precisely, formulas which hold for the Lévy model continue to hold for the others, once appropriate (matrix) scale functions are identified.

We will call this body of related first passage formulas the scale functions kit or cookbook. Its availability means that the analytic work required to solve a first passage problem may often be replaced by looking up in the cookbook. The next section contains ten of our favorite recipes.

\section{TEN FIRST PASSAGE LAWS}

We will start with the easiest problem, which involves only $W_{q}(\cdot)$.

\subsection{Expected discounted dividends}

We review now expected discounted dividends $U$ under both reflection and absorption regimes. These are especially important in the control of reserves processes - see Section 9.

Theorem 6.1. A) The expected total discounted dividends up to $T_{0}^{b]}$ are given by

$$
V^{b]}(x):=\mathbb{E}_{x}^{\mid 0, b]}\left[\int_{\left[0, T_{0}^{b]}\right]} e^{-q t} \mathrm{~d} U_{t}\right]=\frac{W_{q}(x)}{W_{q}^{\prime}(b)},
$$

where $\mathbb{E}^{[0, b]}$ denotes the law of the process reflected from above at $b$, and absorbed at 0 and below.

B) The expected total discounted dividends over an infinite horizon for the doubly reflected process, with expectation denoted $\mathbb{E}^{[0, b]}$, are given by ([31], (4.3))

$$
V^{[0, b]}(x):=\mathbb{E}_{x}^{[0, b]}\left[\int_{0}^{\infty} e^{-q t} \mathrm{~d} U_{t}\right]=\frac{Z_{q}(x)}{Z_{q}^{\prime}(b)} .
$$

Proof. A) Since $V^{b]}(x)=\frac{W_{q}(x)}{W_{q}(b)} V^{b]}(b)$ by the smooth-exit law (3.5), the essential part is proving the result for $x=b$, i.e. that $V^{b]}(b)=\frac{W_{q}(b)}{W_{q}^{\prime}(b)}=\nu_{q}(b)^{-1}$, where the latter (excursion theoretic) quantity has already been introduced in Remark 3.3. For the Cramér-Lundberg case, a direct computation of $V^{b]}(b)$ is provided in Lemma 6.4 in [93]; for the spectrally negative case, a generalization to all moments of the discounted dividends (using excursion theory) may be found in Theorem 10.3 in [94].

To see the idea behind the excursion theory proof, note, following [6], that

$$
\mathbb{E}_{x}^{b]}\left[\int_{0}^{T_{0}^{b]}} e^{-q t} \mathrm{~d} U_{t}\right]=\mathbb{E}_{x}^{b]}\left[\int_{0}^{T_{0}^{b]} \wedge e_{q}} \mathrm{~d} U_{t}\right]=\mathbb{E}_{x}^{b]}\left[U_{T_{0}^{b]} \wedge e_{q}}\right]
$$

Finally, the law of variable $U_{T_{0}^{b]} \wedge e_{q}} \mid x=b$ is exponential with parameter $\nu_{q}(b)$, cf. Remark 3.3 (see also Thm. 6.8 A) below for a generalization).

\footnotetext{
${ }^{14}$ For the construction of these, one may use a recursive approach, or the recent paper [87].
} 
B) Again, it is enough to prove the result for $x=b$, since

$$
\begin{aligned}
V^{[0, b]}(x) & =\mathbb{E}_{x}^{[0, b]}\left[\int_{0}^{\infty} e^{-q t} \mathrm{~d} U_{t}\right]=\mathbb{E}_{x}^{[0, b]}\left[\int_{T_{b}^{[0}}^{\infty} e^{-q t} \mathrm{~d} U_{t}\right]=\mathbb{E}_{x}^{[0, b]}\left[e^{-q T_{b}^{[0}} \int_{0}^{\infty} e^{-q t} \mathrm{~d} U_{t}\right] \\
& =\frac{Z_{q}(x)}{Z_{q}(b)} \mathbb{E}_{x}^{b]}\left[\int_{0}^{e_{q}} \mathrm{~d} U_{t}\right]=\frac{Z_{q}(x)}{Z_{q}(b)} \mathbb{E}_{x}^{b]}\left[U_{e_{q}}\right] .
\end{aligned}
$$

It turns out that for $x=b$, the variable $U_{e_{q}}$ under the measure $\mathbb{E}_{x}^{[0, b]}$ is exponential with parameter $\frac{Z_{q}^{\prime}(b)}{Z_{q}(b)}$, yielding the result (see Thm. 6.13 and Rem. 6.14 below for a generalization and further references).

Remark 6.2. Since the boundary condition $V^{b]}(b)=\frac{W_{q}(b)}{W_{q}^{\prime}(b)}$ in A) requires excursion theory, one might try to establish instead the simpler boundary condition on the derivative

$$
\left(V^{b]}\right)^{\prime}(b)=1,
$$

which says roughly that

$$
V^{b]}(b)-V^{b]}(b-\epsilon) \sim \epsilon
$$

1. Let us start with the Cramér-Lundberg model, and follow the derivation suggested in [70], which note that when starting from $b-\epsilon$, no dividends are gained during a period of $\frac{\epsilon}{c}$, while when starting from $b$, dividends roughly equal to $c \frac{\epsilon}{c}=\epsilon$ are gained during this period.

More precisely, construct the processes starting from $b$ and $b-\epsilon$ on the same probability space, and let $A$ denote the event that there is no jump in the interval $[0, \epsilon / c]$. Over this event, the processes are coupled at time $\frac{\epsilon}{c}$ and the only difference between the dividends comes from the interval $[0, \epsilon / c]$. Putting now together the contribution over $A$ and over its complement yields:

$$
\begin{aligned}
& \frac{V^{b]}(b)-V^{b]}(b-\epsilon)}{\epsilon} \\
& =\frac{c}{\epsilon} \int_{0}^{\frac{\epsilon}{c}} e^{-q s} \mathrm{~d} s+\epsilon^{-1} \int_{0}^{\epsilon / c} \lambda e^{-\lambda s-q s} \int_{0}^{b}(V(b+c s-x)-V(b-\epsilon+c s-x)) f(x) \mathrm{d} x \mathrm{~d} s \\
& \leq \frac{c}{\epsilon} \int_{0}^{\frac{\epsilon}{c}} e^{-q s} \mathrm{~d} s+\epsilon^{-1} \int_{0}^{\epsilon / c} \lambda e^{-\lambda s-q s} \int_{0}^{b} \frac{\lambda}{c} V(b+c s-x) \epsilon f(x) \mathrm{d} x \rightarrow 1,
\end{aligned}
$$

where we used the increasingness and locally Lipschitz property of the value function [139] ([36], 1.3, Prop. 1.3, p. 9), in the Cramér-Lundberg case.

2. We turn now to the spectrally negative Lévy model. Armed with our two exit laws, we find:

$$
\begin{aligned}
& V^{b]}(b-\epsilon)=\frac{W_{q}(b-\epsilon)}{W_{q}(b)} V^{b]}(b)+\left(Z_{q}(b-\epsilon)-\frac{W_{q}(b-\epsilon)}{W_{q}(b)} Z_{q}(b)\right) \times 0 \\
& \Leftrightarrow \frac{V^{b]}(b)-V^{b]}(b-\epsilon)}{\epsilon}=\frac{W_{q}(b)-W_{q}(b-\epsilon)}{\epsilon W_{q}(b)} V^{b]}(b) \\
& \Leftrightarrow\left(V^{b]}\right)^{\prime}(b)=\frac{W_{q}^{\prime}(b)}{W_{q}(b)} V^{b]}(b)
\end{aligned}
$$


and we fall back on the problem of tackling $V^{b]}(b)$, suggesting that the boundary condition is not trivial and that the use of excursion theory (see [43]) is unavoidable in general. Note however that the perturbed Cramér-Lundberg model was solved in [96], via a perturbation approach.

\subsection{The total discounted capital injections/bailout law, with non-smooth regulation}

The next result $[78,125]$ shows the importance of $Z$ for reflected spectrally negative Lévy processes. It also provides a generalization of the fundamental survival probability formula (1.3).

Theorem 6.3. The Laplace transform of the discounted capital injections/bailouts for the process reflected below. Let $X_{t}^{[0}$ denote the process reflected at 0 (1.7) with regulator $L_{t}=-\underline{X}_{t}$, let $\mathbb{E}_{x}^{[0}$ denote expectation for this process and let

$$
T_{b}^{[0}=T_{b,+} \mathbb{1}_{\left\{T_{b,+}<T_{0}\right\}}+\underline{\tau}_{b} \mathbb{1}_{\left\{T_{0}<T_{b,+}\right\}}
$$

denote the first passage to $b$ of $X_{t}^{[0}$, to be called "reflected up time". The total capital injections into the process reflected at 0 , until the first up-crossing of a level b satisfy ${ }^{15}$ ([78], Thm. 2):

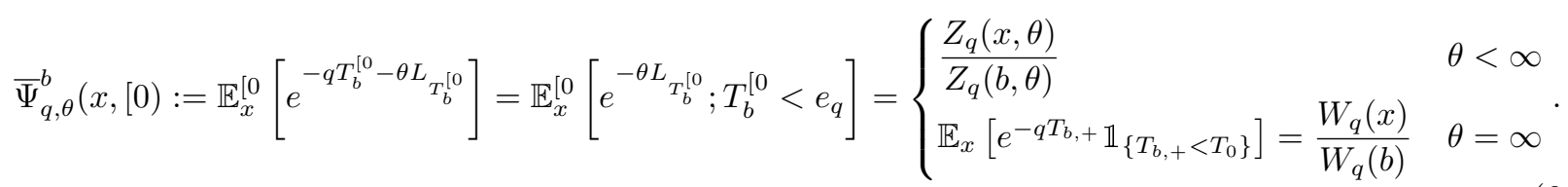

Remark 6.4. Theorem 6.3 was first proved in Theorem 2 from [78] as a consequence of a more general result ([78], Thm. 13), but we prefer to use the observation that it is essentially equivalent to (6.8) [78]. Indeed, (6.5) implies:

$$
\mathbb{E}_{x}^{[0}\left[e^{-q T_{b}^{[0}-\theta L_{T_{b}^{[0}}}\right]=\mathbb{E}_{x}\left[e^{-q T_{0}+\theta X_{T_{0}}} ; T_{0}<T_{b,+}\right] \mathbb{E}_{0}^{[0}\left[e^{-q T_{b}^{[0}-\theta L_{T_{b}}^{[0}}\right]+W_{q}(x) W_{q}(b)^{-1}
$$

If the first term is known one gets an equation for the deficit at ruin

$$
Z_{q}(x, \theta) Z_{q}(b, \theta)^{-1}=W_{q}(x) W_{q}(b)^{-1}+\mathbb{E}_{x}\left[e^{-q T_{0}+\theta X_{T_{0}}} ; T_{0}<T_{b,+}\right] Z_{q}(b, \theta)^{-1},
$$

with the known solution $\mathbb{E}_{x}\left[e^{-q T_{0}+\theta X_{T_{0}}} ; T_{0}<T_{b,+}\right]=Z_{q}(x, \theta)-W_{q}(x) W_{q}(b)^{-1} Z_{q}(b, \theta)$. And if the deficit at ruin is known, one may use (6.7) with $x=0$ to solve for $\mathbb{E}_{0}^{[0}\left[e^{-q T_{b,+}-\theta L\left(T_{b,+}\right)}\right]$, provided that $W_{q}(0) \neq 0$. When $W_{q}(0)=0$, one must start with a "perturbation (approximation) approach", letting $x \rightarrow 0$ [153]- see also Section 8.1 , where this result is proved directly, in the more general context of Parisian ruin.

\subsection{Deficit at ruin}

We turn now to problems of deficit at ruin. We will present here a generalization of the "non-smooth-exit law", featuring the $Z_{q}(x, \theta)$ function.

\footnotetext{
${ }^{15}$ The result (6.6) above may be viewed as the fundamental law of spectrally negative Lévy processes, since it implies the fundamental smooth two-sided exit formula (3.5). Note also that formally, replacing absorption at the boundary 0 by reflection leads to replacing $W$ by $Z$; this will be further confirmed in several of the results below.
} 
Theorem 6.5. Deficit at ruin for a process absorbed or reflected at $b>0$.

A) The joint Laplace transform of the first passage time of 0 and the undershoot for a process absorbed at $b>0$ is given by ([32], Prop. 5.5; [78], Cor. 3; [12], (5))

$$
\Psi_{q, \theta}^{b}(x):=\mathbb{E}_{x}\left[e^{-q T_{0}+\theta X_{T_{0}}} \mathbb{1}_{\left\{T_{0}<T_{b,+}\right\}}\right]=Z_{q}(x, \theta)-\frac{W_{q}(x)}{W_{q}(b)} Z_{q}(b, \theta), x \geq 0 .
$$

B) The joint Laplace transform of the first passage time at 0 ("reflected ruin time", see (4.3)) and the undershoot in the presence of reflection at a barrier $b \geq 0$ is ([32],Prop. 5.5; [78], Thm. 6)

$$
\Psi_{q, \theta}^{b]}(x):=\mathbb{E}_{x}^{b]}\left[e^{-q T_{0}^{b]}+\theta X_{T_{0}^{b]}}^{b]}}\right]=Z_{q}(x, \theta)-\frac{W_{q}(x)}{W_{q}^{\prime}(b)} Z_{q}^{\prime}(b, \theta), x \geq 0
$$

Proof sketch. A) is a consequence of the harmonicity/q-martingale property of $Z_{q}\left(X_{t}, \theta\right)$, and of the boundary condition it satisfies (5.10). Indeed, stopping the martingale $e^{-q t} Z_{q}\left(X_{t}, \theta\right)$ at $\min \left(T_{b,+}, T_{0}\right)$ yields

$$
\begin{aligned}
Z_{q}(x, \theta) & =\mathbb{E}_{x}\left[e^{-q T_{b,+}} Z_{q}(b, \theta) \mathbb{1}_{\left\{T_{b,+}<T_{0}\right\}}\right]+\mathbb{E}_{x}\left[e^{-q T_{0}} Z_{q}\left(X_{T_{0}}, \theta\right) \mathbb{1}_{\left\{T_{0}<T_{b,+}\right\}}\right] \\
& =\frac{W_{q}(x)}{W_{q}(b)} Z_{q}(b, \theta)+\mathbb{E}_{x}\left[e^{-q T_{0}+\theta X_{T_{0}}} \mathbb{1}_{\left\{T_{0}<T_{b,+}\right\}}\right]=\frac{W_{q}(x)}{W_{q}(b)} Z_{q}(b, \theta)+\Psi_{q, \theta}^{b}(x) .
\end{aligned}
$$

Note also that using another (less smooth) harmonic function with the same boundary condition, necessarily of the form $Z_{q}(x, \theta)+k W_{q}(x), k \neq 0$ would not change anything, since $W_{q}(x)$ would cancel in the final result. ${ }^{16}$

B) Conditioning at $\min \left(T_{b,+}, T_{0}\right)$ shows that $\Psi_{q}^{b]}(x, \theta)$ is also of the form $Z_{q}(x, \theta)-k W_{q}(x)$. To determine $k$, we need to use either the (non-trivial) boundary condition $\left(\Psi_{q, \theta}^{b]}\right)^{\prime}(b)=0$ or the final value

$$
\Psi_{q, \theta}^{b]}(b)=Z_{q}(b, \theta)-\frac{W_{q}(b)}{W_{q}^{\prime}(b)} Z_{q}^{\prime}(b, \theta)
$$

The latter has been established in the related drawdown literature - see (6.17) and Theorem 6.7 for a generalization and further references.

Corollary 6.6. A) By using $\lim _{b \rightarrow \infty} \frac{Z_{q}(b, \theta)}{W_{q}(b)}=\frac{\kappa(\theta)-q}{\theta-\Phi(q)}$ (see (13.1) below) in (6.8), we recover ([12], (7))

$$
\mathbb{E}_{x}\left[e^{-q T_{0}+\theta X_{T_{0}}} \mathbb{1}_{\left\{T_{0}<\infty\right\}}\right]=Z_{q}(x, \theta)-W_{q}(x) \frac{\kappa(\theta)-q}{\theta-\Phi(q)}, \quad \theta>\Phi(q) .
$$

B) The relation (6.10) holds as well for $\theta=0$, by analytic continuation, recovering the classic ruin time transform ([22], (10))

$$
\mathbb{E}_{x}\left[e^{-q T_{0}} \mathbb{1}_{\left\{T_{0}<\infty\right\}}\right]=Z_{q}(x)-W_{q}(x) \frac{q}{\Phi(q)}
$$

\footnotetext{
${ }^{16} \mathrm{~A}$ direct proof using the resolvent formula (3.16) and (4.4) is also possible.
} 
C) The limit of (6.10) when $\theta \rightarrow \infty$, which is the second term in the asymptotic expansion (5.1), is

$$
\begin{aligned}
& \lim _{\theta \rightarrow \infty} \mathbb{E}_{x}\left[e^{-q T_{0}+\theta X_{T_{0}}}\right]=\lim _{\theta \rightarrow \infty}\left(Z_{q}(x, \theta)-\frac{\kappa(\theta)-q}{\theta-\Phi_{q}} W_{q}(x)\right) \\
& =\mathbb{E}_{x}\left[e^{-q T_{0}} ; X_{T_{\{0\}}}=0\right]=\frac{\sigma^{2}}{2}\left(W_{q}^{\prime}(x)-\Phi_{q} W_{q}(x)\right) .
\end{aligned}
$$

The last equality is the so-called "creeping law" ([126], Cor. 2; [85], (2.30)).

D) A similar result for the hitting time of 0 ("recovery after ruin") may be obtained by letting first $\theta \rightarrow \Phi_{q}$ in (6.10).

Indeed, using $\kappa^{\prime}\left(\Phi_{q}\right)=\frac{1}{\Phi_{q}^{\prime}}$ and (5.3), we find

$$
\mathbb{E}_{x}\left[e^{-q T_{0}+\Phi_{q} X_{T_{0}}} \mathbb{1}_{\left\{T_{0}<\infty\right\}}\right]=Z_{q}(x, \Phi(q))-\frac{1}{\Phi_{q}^{\prime}} W_{q}(x)=e^{x \Phi_{q}}-\frac{1}{\Phi_{q}^{\prime}} W_{q}(x) .
$$

Turning now to the Laplace transform of the hitting time of 0 , we find that for $x \geq 0$,

$$
\begin{aligned}
& \mathbb{E}_{x}\left[e^{-q T_{\{0\}}} \mathbb{1}_{\left\{T_{\{0\}}<\infty\right\}}\right]=\mathbb{E}_{x}\left[\mathbb{E}_{x}\left[e^{-q T_{0}-q\left(T_{\{0\}}-T_{0}\right)} \mathbb{1}_{\left\{T_{0}<\infty\right\}} \mid X_{T_{0}}\right]\right] \\
& =\mathbb{E}_{x}\left[e^{-q T_{0}+\Phi_{q} X_{T_{0}}} \mathbb{1}_{\left\{T_{0}<\infty\right\}}\right]=e^{x \Phi_{q}}-\frac{1}{\Phi_{q}^{\prime}} W_{q}(x),
\end{aligned}
$$

(alternatively, this formula may be obtained by a martingale stopping argument, and holds for $x \in \mathbb{R}$ as well). This yields the representation of $W_{q}$ announced in (3.13):

$$
e^{x \Phi_{q}}-\frac{W_{q}(x)}{\Phi_{q}^{\prime}}=\mathbb{P}_{x}\left[T_{\{0\}}<e_{q}\right]
$$

\subsection{From drawdowns to the dividends-penalty law}

This section and the following ones will exploit the connection between drawdown s and dividends. Namely, the law of the drawdown triple and that of the dividend triple

$$
\left.\left(\tau_{b}, \bar{X}_{\tau_{b}}-X_{0}, Y_{\tau_{b}}-b,\right) \mid\left\{X_{0}=b\right\}, \quad\left(T_{0}^{b]}, U_{T_{0}^{b]}},-X_{T_{0}^{b]}}\right)\right)
$$

coincide. See Figure 1 below, where the paths of the process $X^{b]}$ are obtained from the paths of the process $X$ on the right by Skorokhod reflection at $b$. For the picture of $X$, we may assume that $X_{0}=b$ for simplicity, but that is not necessary. Now note that: a) the times $T_{0}^{b]}$ and $\tau_{b}$ coincide; b) the total regulation equals the sum of the projections on the $X$ axis of the segments when $X$ is at a running maximum; c) the last drop must be the same on both pictures, since no reflection occurs during the last drop. Thus $b-X_{T_{0}^{b]}}=Y_{\tau_{b}} .{ }^{17}$

This section reviews first the independence of the law of the supremum $\bar{X}_{\tau_{d}}-x$ of the law of the (killed) drawdown achieved on the last downwards excursion. The former law is exponential with parameter $\nu_{q}(d)=$ $\frac{W_{q}^{\prime}(d)}{W_{q}(d)}$ (recall this follows intuitively from the fact that the upward ladder process with downward excursions excised is a drift killed at rate $\nu_{q}(d)$ ). The independence is due intuitively to the fact that each time the upward ladder process reaches a new point, the search for the killing excursion larger than $d$ starts again.

\footnotetext{
${ }^{17}$ To understand Skorokhod reflection informally, imagine the process $X$ arrives to $b$ from below, and encounters a barrier. If the barrier is fixed, it is forced to stick to the barrier until the first impulse downwards. If the barrier is movable, it is just raised during running maximum periods. In physics, under these two hypotheses, $b-X_{t}$ represents the distance to $b$ with respect to a fixed and moving frame, respectively.
} 

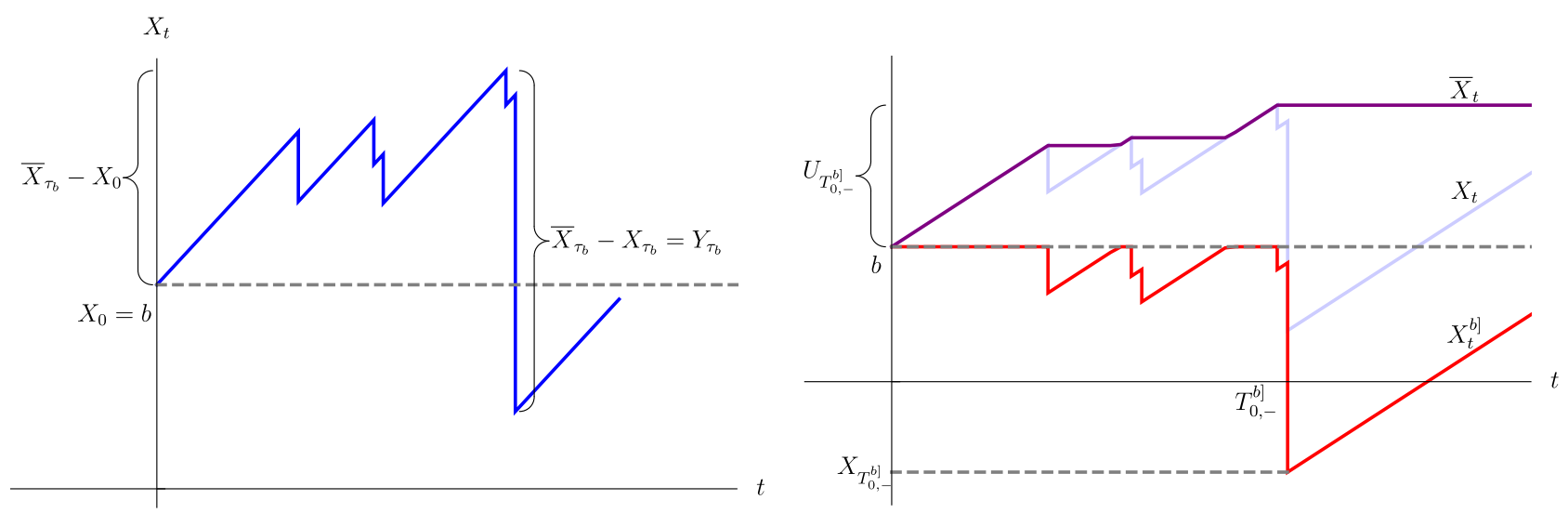

FiguRe 1. Drawdown and dividend triples (6.15).

Equivalently, by (6.15), the independence of the dividends until ruin and of the final deficit when starting from $b$ follows. When starting from $x<b$, one gets the famous Dividends-Penalty identity first obtained in [111].

Theorem 6.7. The deficit at drawdown [118] ([99], Thm. 3.1) ([110], Props. 3.1, 3.2) $)^{18}$ satisfies:

$$
\begin{aligned}
& \delta_{q, \theta}(d, x, s):=\mathbb{E}_{x}\left[e^{-q \tau_{d}-\theta\left(Y_{\tau_{d}}-d\right)} ; \bar{X}_{\tau_{d}} \in \mathrm{d} s\right]=\left(\nu_{q}(d) e^{-\nu_{q}(d)(s-x)_{+}} d s\right) \widetilde{\delta}_{q, \theta}(d) \\
& \Leftrightarrow \mathbb{E}_{x}\left[e^{-q \tau_{d}-\theta\left(Y_{\tau_{d}}-d\right)-\vartheta\left(\bar{X}_{\tau_{d}}-x\right)}\right]=\frac{\nu_{q}(d)}{\vartheta+\nu_{q}(d)} \widetilde{\delta}_{q, \theta}(d)
\end{aligned}
$$

where

$$
\widetilde{\delta}_{q, \theta}(d)=E_{x}\left[e^{-q \tau_{d}-\theta\left(Y_{\tau_{d}}-d\right)}\right]=Z_{q}(d, \theta)-W_{q}(d) \frac{Z_{q}^{\prime}(d, \theta)}{W_{q}^{\prime}(d)} .
$$

Using now the alternative interpretation furnished by (6.15) yields a powerful generalization of the deficit at ruin with reflection, Theorem $6.5 \mathrm{~B}$ ):

Theorem 6.8. Let

$$
D P_{q, \theta, \vartheta}^{b]}(x):=\mathbb{E}_{x}^{b]}\left[e^{-q T_{0}^{b]}+\theta X_{T_{0}^{b]}}-\vartheta U_{T_{0}^{b]}}}\right]
$$

denote the dividends-penalty Laplace transform. ${ }^{19}$

A) When $x=b$, it holds that

$$
D P_{q, \theta, \vartheta}^{b]}(b)=\frac{\nu_{q}(b)}{\vartheta+\nu_{q}(b)} \widetilde{\delta}_{q, \theta}(b) .
$$

Thus, when starting from $x=b$, the dividends $U_{T_{0}^{b]} \wedge e_{q}}$ and the deficit at ruin $X_{T_{0}^{b]} \wedge e_{q}}$ are independent, with the first variable having an exponential distribution [94].

\footnotetext{
${ }^{18}$ We have re-expressed the result using the transformations in Remark 5.4.

${ }^{19}$ On an arbitrary interval $\left.\mid a, b\right]$, we will use the notation $\Psi_{q, \theta, \vartheta}^{b]}(x, a)$.
} 
B) Furthermore ([78], Thm. 6):

$$
\begin{aligned}
& D P_{q, \theta, \vartheta}^{b]}(x)=Z_{q}(x, \theta)-W_{q}(x) H_{D P}(b), \\
& H_{D P}(b)=\frac{Z_{q}^{\prime}(b, \theta)+\vartheta Z_{q}(b, \theta)}{W_{q}^{\prime}(b)+\vartheta W_{q}(b)} .
\end{aligned}
$$

Proof. A) When starting at $x=b$ one may apply Theorem 6.7 from the drawdown literature. ${ }^{20}$

B) Stopping at $T_{0} \wedge T_{b,+}$ yields that $l(x)$ satisfies:

$$
l(x)=Z_{q}(x, \theta)-\frac{W_{q}(x)}{W_{q}(b)} Z_{q}(b, \theta)+\frac{W_{q}(x)}{W_{q}(b)} l(b)=Z_{q}(x, \theta)+W_{q}(x) \frac{l(b)-Z_{q}(b, \theta)}{W_{q}(b)}
$$

and the result follows from part A) by easy algebra.

Remark 6.9. It is easy to check that when $x=b$, the transform (6.19) factorizes and we recover (6.18):

$$
\begin{aligned}
D P_{q, \theta, \vartheta}^{b]}(b) & =\frac{Z_{q}(b, \theta) W_{q}^{\prime}(b)-Z_{q}^{\prime}(b, \theta) W_{q}(b)}{W_{q}^{\prime}(b)+\vartheta W_{q}(b)} \\
& =\frac{\frac{W_{q}^{\prime}(b)}{W_{q}(b)}}{\frac{W_{q}^{\prime}(b)}{W_{q}(b)}+\vartheta}\left(Z_{q}(b, \theta)-Z_{q}^{\prime}(b, \theta) \frac{W_{q}(b)}{W_{q}^{\prime}(b)}\right)=\frac{\frac{W_{q}^{\prime}(b)}{W_{q}(b)}}{\frac{W_{q}^{\prime}(b)}{W_{q}(b)}+\vartheta} \widetilde{\delta}_{q, \theta}(b) .
\end{aligned}
$$

Remark 6.10. Setting $\vartheta=0$ in $D P_{q, \theta, \vartheta}^{b]}(b)$ yields

$$
D P_{q, \theta, 0}^{b]}(b)=\delta_{q, \theta}(b)=\Psi_{q, \theta}^{b]}(b)=\frac{\Delta_{q, \theta}^{(Z W)}(b)}{W_{q}^{\prime}(b)} \in(0,1)
$$

where we denoted

$$
\Delta_{q, \theta}^{(Z W)}(x, b):=Z_{q}(x, \theta) W_{q}^{\prime}(b)-Z_{q}^{\prime}(b, \theta) W_{q}(x), \Delta_{q, \theta}^{(Z W)}(b)=\Delta_{q, \theta}^{(Z W)}(b, b) .
$$

The obvious nonnegativity of $\Delta_{q, \theta}^{(Z W)}$ implies that the function $\frac{Z_{q}(b, \theta)}{W_{q}(b)}$ is decreasing (other papers refer to this as the log-convexity of $\left.Z_{q}(x)\right)$. It also implies an upper bound for the Wronskian

$$
\left.0 \leq \Delta^{(\bar{W} W)}:=W_{q}^{2}(b)-\bar{W}_{q}(b) W_{q}(b)\right) \leq q^{-1} W_{q}^{\prime}(b) .
$$

\subsection{From bailouts to the joint dividends-bailouts law}

After dividends, we now turn to bailouts as defined by $L_{t}=-\min \left(\underline{X}_{t}, 0\right)$, and finally to their joint law.

\section{Theorem 6.11. Bailouts until an exponential time.}

$$
\text { A) } \mathbb{E}_{x}^{[0}\left[e^{-\theta L_{e_{q}}} ; e_{q}<T_{b}^{[0}\right]=1-Z_{q}(x)-Z_{q}(x, \theta) \frac{1-Z_{q}(b)}{Z_{q}(b, \theta)}
$$

\footnotetext{
${ }^{20}$ Putting $l(x):=D P_{q, \theta, \vartheta}^{b]}(x)$, the (mixed) boundary condition at $x=b$ is now $l^{\prime}(b)+\vartheta l(b)=0$; this offers another line of attack, at least in the Cramér-Lundberg case.
} 


$$
\begin{aligned}
& \text { B) } \mathbb{E}_{x}^{[0}\left[e^{\left.-\theta L_{e_{q} \wedge T_{b}^{[0}}\right]}=1-Z_{q}(x)+Z_{q}(x, \theta) \frac{Z_{q}(b)}{Z_{q}(b, \theta)}\right. \\
& \text { C) } \mathbb{E}_{x}^{[0, b]}\left[e^{\left.-\theta L_{e_{q}}\right]}=1-Z_{q}(x)+Z_{q}(x, \theta) \frac{Z_{q}^{\prime}(b)}{Z_{q}^{\prime}(b, \theta)} .\right.
\end{aligned}
$$

Proof. A) Decompose $l(x):=\mathbb{E}_{x}^{[0}\left[e^{-\theta L_{e_{q}}} ; e_{q}<T_{b}^{[0]}\right]$ as

$$
\begin{aligned}
l(x) & \left.=\mathbb{E}_{x}^{[0}\left[e^{-\theta L_{e_{q}}} ; e_{q}<T_{0} \wedge T_{b,+}\right]+\mathbb{E}_{x}^{[0}\left[e^{-\theta L_{e_{q}}} ; T_{0} \leq e_{q}<T_{b,+}\right)\right] \\
& =P_{x}^{[0}\left[e_{q}<T_{0} \wedge T_{b,+}\right]+\mathbb{E}_{x}^{[0}\left[e^{\theta X_{T_{0}}} ; T_{0} \leq e_{q} \wedge T_{b,+}\right] \mathbb{E}_{0}^{[0}\left[e^{-\theta L_{e_{q}}} ; e_{q}<T_{b}^{[0}\right] \\
& =\left(1-Z_{q}(x)+\frac{W_{q}(x)}{W_{q}(b)}\left(Z_{q}(b)-1\right)\right)+\left(Z_{q}(x, \theta)-\frac{W_{q}(x)}{W_{q}(b)} Z_{q}(b, \theta)\right) l(0),
\end{aligned}
$$

where we used the minimum law $(4.7)$ and the deficit law (6.8). In the Cramér-Lundberg case when $W_{q}(0) \neq 0$ we may plug $x=0$ and conclude that

$$
l(0)=\frac{q \bar{W}(b)}{Z_{q}(b, \theta)} .
$$

The same may be shown in the general case by a perturbation argument. Plugging now $l(0)$ yields the result A).

B) Follows by adding (6.6).

C) Follows by conditioning at time $e_{q} \wedge T_{b}^{[0}$, where $h(x):=\mathbb{E}_{x}^{[0, b]}\left[e^{-\theta L_{e_{q}}}\right]$. Indeed,

$$
\begin{aligned}
& h(x)=\left(1-Z_{q}(x)+Z_{q}(x, \theta) \frac{Z_{q}(b)-1}{Z_{q}(b, \theta)}\right)+\frac{Z_{q}(x, \theta)}{Z_{q}(b, \theta)} h(b) \\
& \Longrightarrow \frac{h(x)+Z_{q}(x)-1}{Z_{q}(x, \theta)}=\frac{h(b)+Z_{q}(b)-1}{Z_{q}(b, \theta)}=\frac{Z_{q}^{\prime}(b)}{Z_{q}^{\prime}(b, \theta)}, \forall x,
\end{aligned}
$$

where for the last equality we have used $h^{\prime}(b)=0$ and the fact that for two functions $f$ and $g, f(x) / g(x)=c$ implies $f^{\prime}(x) / g^{\prime}(x)=c$.

Remark 6.12. By letting $b \rightarrow \infty$ in B) we recover ([7], Lem. 3.1).

Theorem 6.13. The joint dividends-bailouts law for a process doubly reflected at 0 and b, over an exponential horizon.

The dividends-bailouts function is given by

$$
\begin{aligned}
& D B_{q}^{[0, b]}(x, \theta, \vartheta):=\mathbb{E}_{x}^{[0, b]}\left[e^{\left.-\vartheta U_{e_{q}}-\theta L_{e_{q}}\right]}=1-Z_{q}(x)+Z_{q}(x, \theta) D B_{q}^{[0, b]}(0, \theta, \vartheta),\right. \\
& D B_{q}^{[0, b]}(0, \theta, \vartheta)=\frac{Z_{q}^{\prime}(b)+\vartheta\left(Z_{q}(b)-1\right)}{\left.Z_{q}^{\prime}(b, \theta)+\vartheta Z_{q}(b, \theta)\right)}=q \frac{W_{q}(b)+\vartheta \bar{W}_{q}(b)}{\left.Z_{q}^{\prime}(b, \theta)+\vartheta Z_{q}(b, \theta)\right)}:=H_{D B}(b) .
\end{aligned}
$$


Proof. Conditioning at $e_{q} \wedge T_{b}^{[0}$ and using Theorem $\left.6.11 \mathrm{~A}\right)$ and Theorem 6.3 we find

$$
\begin{aligned}
l(x) & :=D B_{q}^{[0, b]}(x, \theta, \vartheta)=\mathbb{E}_{x}^{[0, b]}\left[e^{-\theta L_{e_{q}}} ; e_{q}<T_{b}^{[0]}\right]+\mathbb{E}_{x}^{[0, b]}\left[e^{-\theta L_{T_{b}}^{[0} ; T_{b}^{[0}} \leq e_{q}\right] l(b) \\
& =1-Z_{q}(x)-\frac{Z_{q}(x, \theta)}{Z_{q}(b, \theta)}\left(1-Z_{q}(b)\right)+\frac{Z_{q}(x, \theta)}{Z_{q}(b, \theta)} l(b) \\
& \Longrightarrow \frac{l(x)-1+Z_{q}(x)}{Z_{q}(x, \theta)}=\frac{l(b)-1+Z_{q}(b)}{Z_{q}(b, \theta)}=l(0) .
\end{aligned}
$$

The value of $l(b)$

$$
l(b)=\mathbb{E}_{b}^{[0, b]}\left[e^{-\vartheta U_{e_{q}}-\theta L_{e_{q}}}\right]=\frac{Z_{q}^{\prime}(b, \theta)+\left(Z_{q}(b, \theta) Z_{q}^{\prime}(b)-Z_{q}^{\prime}(b, \theta) Z_{q}(b)\right)}{Z_{q}^{\prime}(b, \theta)+\vartheta Z_{q}(b, \theta)}
$$

was obtained in Theorem 1 in [7], via excursion theoretic arguments.

Remark 6.14. When $\theta=0,(6.24)$ shows that discounted dividends starting from $b$ over an exponential horizon, with double reflection, have an exponential law with parameter $\frac{Z_{q}^{\prime}(b)}{Z_{q}(b)}$, a surprising result which seems to have gone unnoticed. Also, $\mathbb{E}_{x}^{[0, b]}\left[e^{\left.-\vartheta U_{e_{q}}\right]}=1-\frac{\vartheta Z_{q}(x)}{Z_{q}^{\prime}(b)+\vartheta Z_{q}(b)}\right.$, recovering $\mathbb{E}_{x}^{[0, b]}\left[U_{e_{q}}\right]=\frac{Z_{q}(x)}{Z_{q}^{\prime}(b)}([31],(4.3))$.

Putting $\vartheta=0$ in $(6.23)$ yields Theorem $6.11 \mathrm{C})$, and differentiating recovers $([31],(4.4))$

$$
\begin{aligned}
\mathbb{E}_{x}^{[0, b]}\left[L_{e_{q}}\right] & =\frac{1}{Z_{q}^{\prime}(b)}\left[Z_{q}(x)\left(Z_{q}(b)-\kappa^{\prime}\left(0_{+}\right) W_{q}(b)\right)-\left(\bar{Z}_{q}(x)-\kappa^{\prime}\left(0_{+}\right) \bar{W}_{q}(x)\right) q W_{q}(b)\right] \\
& =\frac{Z_{q}(x) Z_{q}(b)-\bar{Z}_{q}(x) Z_{q}^{\prime}(b)-\kappa^{\prime}\left(0_{+}\right) W_{q}(b)}{Z_{q}^{\prime}(b)}=\frac{Z_{q}(x) Z_{q}(b)}{Z_{q}^{\prime}(b)}-\bar{Z}_{q}(x)-\frac{\kappa^{\prime}\left(0_{+}\right)}{q}
\end{aligned}
$$

where $\bar{Z}_{q}(x)$ is defined in (5.5).

\subsection{Expected discounted bailouts}

We recall now results on expected discounted bailouts until $T_{b,+}$ and over an infinite horizon, which may be obtained simply by differentiating the corresponding moment generating functions in Theorem 6.11 B), C).

Theorem 6.15. Put

$$
G_{q}^{B}(x)=Z_{q}^{(1)}(x)={\frac{\partial Z_{q}(x, \theta)}{\partial \theta}}_{\theta=0}=\bar{Z}_{q}(x)-\kappa^{\prime}\left(0_{+}\right) \bar{W}_{q}(x) .
$$

A) The expectation of the total discounted bailouts up to $T_{b,+}$ for $0 \leq x \leq b$ is ([33], Cor. 3.2 (ii)):

$$
B^{b}(x):=\mathbb{E}_{x}^{[0}\left[\int_{0}^{T_{b}^{[0}} e^{-q t} \mathrm{~d} L_{t}\right]=\mathbb{E}_{x}^{[0}\left[L_{T_{b}^{[0} \wedge e_{q}}\right]=\frac{Z_{q}(x)}{Z_{q}(b)} G_{q}^{B}(b)-G_{q}^{B}(x) .
$$

B) The expected total discounted bailouts over an infinite horizon, with reflection at $b$ are ([31], $(4.4)):$

$$
B^{[0, b]}(x)=\mathbb{E}_{x}^{[0, b]}\left[\int_{0}^{\infty} e^{-q t} \mathrm{~d} L_{t}\right]=\mathbb{E}_{x}^{[0, b]}\left[L_{e_{q}}\right]=\frac{Z_{q}(x)}{Z_{q}^{\prime}(b)}\left(G_{q}^{B}\right)^{\prime}(b)-G_{q}^{B}(x) .
$$


$G_{q}^{B}$ may also be taken to be

$$
G_{q}^{B}(x)=\bar{Z}_{q}(x)+\frac{\kappa^{\prime}\left(0_{+}\right)}{q},
$$

in both results.

Remark 6.16. As may be easily checked, the first expression for $G_{q}^{B}$, i.e. $Z_{q}^{(1)}(x)$, is the smooth Gerber-Shiu function (see [32] and next section), fitting the value of $w(x)=x$ at 0 , and also its derivative in the noncompound Poisson case. Without smoothness, the Gerber-Shiu function is unique only up to adding a multiple of the corresponding scale function, and simpler expressions like (6.28) may be available.

Remark 6.17. Note that several relations for the process reflected below like (6.27), and the relation $\mathbb{E}_{x}^{[0}\left[e^{-q T_{b,+}}\right]=\frac{Z_{q}(x)}{Z_{q}(b)}[22]$ may be obtained formally from analog relations for the process absorbed at 0 , by substituting the second scale function $Z_{q}$ instead of the first scale function $W_{q}$.

\subsection{Results obtained by differentiating the moment generating functions}

We turn now to obtain the expectations of the ruin time, exit time from an interval, reflected ruin time, reflected up time and recovery after ruin time, obtained by differentiating the respective moment generating functions (6.11), (4.7), (6.9), (6.6), (6.14) with respect to $q$ (making use of the analyticity of $W_{q}$ in $q$ [94], Lem. 8.3), and putting $q=0$. In the proof of B) below, we additionally use the fact that when some function $f$ is differentiable at 0 , it holds that $\frac{\partial[q f(q)]}{\partial q}_{q=0}=f(0)$.

Theorem 6.18. A) When $\kappa^{\prime}\left(0_{+}\right)<0 \Longrightarrow \Phi(0)>0$, it holds that

$$
\mathbb{E}_{x}\left[T_{0}\right]=\frac{W(x)}{\Phi(0)}-\bar{W}(x)
$$

When $\kappa^{\prime}\left(0_{+}\right)>0 \Longrightarrow \Phi(0)=0$, it holds that

$$
\begin{aligned}
\mathbb{E}_{x}\left[T_{0} \mathbb{1}_{\left\{T_{0}<\infty\right\}}\right] & =W(x) \lim _{q \rightarrow 0} \frac{\Phi_{q}-q \Phi^{\prime}(q)}{\Phi_{q}^{2}}+\kappa^{\prime}\left(0_{+}\right) W^{* 2}(x)-\bar{W}(x) \\
& =-\kappa^{\prime}\left(0_{+}\right)^{2} \frac{\Phi^{\prime \prime}\left(0_{+}\right)}{2} W(x)+\kappa^{\prime}\left(0_{+}\right) W^{* 2}(x)-\bar{W}(x) \\
& =\frac{\kappa^{\prime \prime}\left(0_{+}\right)}{2 \kappa^{\prime}\left(0_{+}\right)} W(x)+\kappa^{\prime}\left(0_{+}\right) W^{* 2}(x)-\bar{W}(x),
\end{aligned}
$$

where we used

$$
\Phi^{\prime \prime}(x)=-\frac{\kappa^{\prime \prime}(x)}{\left(\kappa^{\prime}(x)\right)^{3}}
$$

and the series expansion ([94], (8.29))

$$
W_{q}(x)=\sum_{k=0}^{\infty} q^{k} W^{*, k+1}(x),
$$

with $W^{*, k}(x)$ denoting convolution. 
B) Put $T=T_{0} \wedge T_{b,+}$. Then ${ }^{21}$

$$
\mathbb{E}_{x}[T]=\frac{W(x)}{W(b)} \bar{W}(b)-\bar{W}(x)
$$

C)

$$
\mathbb{E}_{x}^{b]}\left[T_{0}^{b]}\right]=W(x) \frac{W(b)}{W^{\prime}(b)}-\bar{W}(x) \Longrightarrow \mathbb{E}\left[\tau_{b}\right]=\frac{W(b)^{2}}{W^{\prime}(b)}-\bar{W}(b) .
$$

D)

$$
E\left[T_{b}^{[0]}\right]=\bar{W}(b)
$$

E)

$$
\mathbb{E}_{x}\left[T_{\{0\}} ; T_{\{0\}}<\infty\right]=\kappa^{\prime}(\Phi(0)) W^{*, 2}(x)+\frac{\kappa^{\prime \prime}(\Phi(0))}{\kappa^{\prime}(\Phi(0))} W(x)-\frac{x e^{x \Phi(0)}}{\kappa^{\prime}(\Phi(0))}
$$

When $\kappa^{\prime}\left(0_{+}\right)>0 \Longrightarrow \Phi(0)=0$, this simplifies to

$$
\mathbb{E}_{x}\left[T_{\{0\}} ; T_{\{0\}}<\infty\right]=\kappa^{\prime}\left(0_{+}\right) W^{*, 2}(x)+\frac{\kappa^{\prime \prime}\left(0_{+}\right)}{\kappa^{\prime}\left(0_{+}\right)} W(x)-\frac{x}{\kappa^{\prime}\left(0_{+}\right)} .
$$

Remark 6.19. In the particular compound Poisson case, A) reduces, using $W(x)=\frac{\bar{\Psi}(x)}{\kappa^{\prime}\left(0_{+}\right)}$and $\kappa^{\prime \prime}(0)=\lambda \mathbb{E}\left[C_{i}^{2}\right]$ to $([136],(11.3 .26))$

$$
\mathbb{E}_{x}\left[T_{0} \mathbb{1}_{\left\{T_{0}<\infty\right\}}\right]=\frac{\kappa^{\prime \prime}(0)}{2 \kappa^{\prime}\left(0_{+}\right)^{2}} \bar{\Psi}(x)-\frac{1}{\kappa^{\prime}\left(0_{+}\right)} \int_{0}^{x} \bar{\Psi}(y) \Psi(x-y) \mathrm{d} y
$$

Our examples show that the expected time to ruin conditioning on ruin happening is unimodular, with a unique maximum. This maximum could be viewed as a reasonable lower bound for the initial reserve, which postpones ruin as much as possible (in the worst case).

Remark 6.20. To show the nonnegativity of $\mathrm{C}$ ), it suffices to take $x=b$, where the nonnegativity holds by the log-concavity of $\bar{W}^{(q)}$, proved in Remark 4.5.

When $b \rightarrow \infty$ and $\left.\kappa^{\prime}\left(0_{+}\right)<0, \mathrm{C}\right)$ converges to $\left.\mathrm{A}\right)$.

When $x=0, \mathrm{C})$ yields the "0-cycle law" ([138], Prop. 3.2(i))

$$
\mathbb{E}_{0}^{b]}\left[T_{0}^{b]}\right]=W(0) \frac{W(b)}{W^{\prime}(b)} .
$$

To give an idea of very recent developments in the $W, Z$ theory, we end this section with a hitting time result which holds for certain Omega spectrally negative Markov processes as well ([114], Cor. 1) (the proof is quite elegant).

\footnotetext{
${ }^{21}$ This provides a third proof of the monotonicity of $\frac{\bar{W}(b)}{W(b)}$ (see Rem. 4.5).
} 
Theorem 6.21. For $x, i \in(a, b)$, it holds that

$$
\mathbb{E}_{x}\left[e^{-\int_{0}^{T_{\{i\}}} q \mathrm{~d} s} ; T_{\{i\}} \leq T_{a,-} \wedge T_{b,+}\right]=\frac{W_{q}(x-a)}{W_{q}(i-a)}-\frac{W_{q}(x-i)}{W_{q}(b-i)} \frac{W_{q}(b-a)}{W_{q}(i-a)}
$$

For the general result with Omega non-constant killing, it suffices to replace $\int_{0}^{T_{\{i\}}} q \mathrm{~d} s$ by $\int_{0}^{T_{\{i\}}} \omega\left(X_{s}\right) \mathrm{d} s$, where $\omega: \mathbb{R} \rightarrow \mathbb{R}_{+}$is an arbitrary locally bounded nonnegative measurable state dependent discounting, to replace $b-a$ by $b, a, \ldots$, etc., and to identify the scale function $W_{\omega}[104,114]$ - see also Section 8.2.

\section{Smooth Gerber-Shiu functions: $Z_{q}(x, \theta)$ is RePlaced By The Smooth Gerber-Shiu FunCtion $G_{w}(x)$}

When $e^{\theta X_{T_{0}}}$ is replaced in the previous formulas (6.8), (6.9) by an arbitrary penalty function $w\left(X_{T_{0}}\right), w$ : $(-\infty, 0] \rightarrow \mathbb{R}$, extensions of these formulas still hold for

$$
\mathcal{V}^{b}(x):=\mathbb{E}_{x}\left[e^{-q T_{0}} w\left(X_{T_{0}}\right) \mathbb{1}_{\left\{T_{0}<T_{b,+}\right\}}\right]
$$

if one replaces $Z_{q}(x, \theta)$ by an infinite horizon Gerber-Shiu penalty function

$$
\mathcal{V}(x):=\mathbb{E}_{x}\left[e^{-q T_{0}} w\left(X_{T_{0}}\right)\right]
$$

Indeed, applying the strong Markov property at $T_{b,+}$ immediately yields

$$
\mathcal{V}(x)=\mathcal{V}^{b}(x)+\frac{W_{q}(x)}{W_{q}(b)} \mathcal{V}(b) \Longrightarrow \mathcal{V}^{b}(x)=\mathcal{V}(x)-\frac{W_{q}(x)}{W_{q}(b)} \mathcal{V}(b)
$$

Note that $\mathcal{V}(x)$ is not unique: it may be replaced in the identity above by adding to it any multiple of $W_{q}(x)$ ([32], Prop. 5.4).

For this reason, Theorem 5.3 in [32] identify the unique "smooth Gerber-Shiu function" $G$ ([32], Def. 5.2), which exists if $w$ satisfies some minimal integrability conditions. Under these, given $0<b<\infty, x \in(0, b)$, there exists a unique smooth function $G=G_{q}$ so that the following hold:

$$
\begin{aligned}
& \mathcal{V}^{b}(x)=\mathbb{E}_{x}\left[e^{-q T_{0}} w\left(X_{T_{0}}\right) 1_{\left\{T_{0}<T_{b,+}\right\}}\right]=G(x)-\frac{W_{q}(x)}{W_{q}(b)} G(b), \\
& \mathcal{V}^{b]}(x)=\mathbb{E}_{x}^{b]}\left[e^{-q T_{0}^{b]}} w\left(X_{T_{0}^{b]}}\right)\right]=G(x)-\frac{W_{q}(x)}{W_{q}^{\prime}(b)} G^{\prime}(b) .
\end{aligned}
$$

Stated informally, both problems above admit decompositions involving the same "non-homogeneous solution" $G$.

The "smoothness" required is:

$$
\left\{\begin{array}{l}
G(0)=w(0), \\
G^{\prime}\left(0_{+}\right)=w^{\prime}\left(0_{-}\right), \quad \text { in the case } \sigma^{2}>0 \text { or } \Pi([0,1])=\infty .
\end{array}\right.
$$


Under these conditions, the function $G$ is unique. Furthermore, it may be represented as ([32], (5.13) Lem. 5.6):

$$
\begin{aligned}
G(x) & =w(0) Z_{q}(x)+w^{\prime}(0-) \frac{\sigma^{2}}{2} W_{q}(x)+\int_{0}^{x} W_{q}(x-y) \int_{z=y}^{\infty}[w(0)-w(y-z)] \Pi(\mathrm{d} z) \mathrm{d} y \\
& =w(0)\left(\frac{\sigma^{2}}{2} W_{q}^{\prime}(x)+c W_{q}(x)\right)+w^{\prime}(0-) \frac{\sigma^{2}}{2} W_{q}(x)-\int_{0}^{x} W_{q}(x-y) w^{(\Pi)}(y) \mathrm{d} y
\end{aligned}
$$

where $w^{(\Pi)}(y)=\int_{z=y}^{\infty}[w(y-z)] \Pi(\mathrm{d} z)$ is the expected liquidation cost conditioned on a pre-ruin position of $y$, with ruin causing jump bigger than $y$. The second equality follows by using (5.4).

Remark 7.1. The last term in the second equality in (7.4) fits the "non-local" part of $w$, and the first two terms may be viewed as boundary fitting terms. Indeed, this holds since $\frac{\sigma^{2}}{2} W_{q}^{\prime}\left(0_{+}\right)+c W_{q}\left(0_{+}\right)=1, \frac{\sigma^{2}}{2} W_{q}\left(0_{+}\right)=0$, and $\frac{\sigma^{2}}{2} W_{q}^{\prime \prime}\left(0_{+}\right)+c W_{q}^{\prime}\left(0_{+}\right)=0, \frac{\sigma^{2}}{2} W_{q}^{\prime}\left(0_{+}\right)=1$.

Proposition 7.2. For $w(x)=e^{\theta x}$, the Gerber-Shiu function is $Z_{q}(x, \theta)$ and the decomposition (7.4) becomes:

$$
Z_{q}(x, \theta)=Z_{q}(x)+\theta \frac{\sigma^{2}}{2} W_{q}(x)+\int_{0}^{x} W_{q}(y) \int_{x-y}^{\infty}\left[1-e^{\theta(x-y-z)}\right] \Pi(\mathrm{d} z) d y .
$$

This may be easily checked by taking Laplace transforms, since

$$
\widehat{W}_{q}(s) \frac{\kappa(s)-\kappa(\theta)}{s-\theta}=\widehat{W}_{q}(s)\left(\frac{\kappa(s)}{s}+\theta \frac{\sigma^{2}}{2}+\frac{\widehat{\pi}(s)-\widehat{\pi}(\theta)}{s-\theta}-\frac{\widehat{\pi}(s)-\widehat{\pi}(0)}{s}\right) .
$$

\section{Poissonian/Parisian detection of Bankruptcy/Insolvency, AND OCCUPATION TIMES}

A useful type of models developed recently $[5,12,33]$ assume that insolvency is only observed periodically, at an increasing sequence of Poisson observation times $\mathcal{T}_{\lambda}=\left\{t_{i}, i=1,2, \ldots\right\}$, the arrival times of an independent Poisson process of rate $\lambda$, with $\lambda>0$ fixed. ${ }^{22}$ The analog concepts for first passage times are the stopping times

$$
T_{b,+}=T_{b,+}^{\lambda}=\inf \left\{t_{i}: X_{t_{i}}>b\right\}, \quad T_{a,-}=T_{a,-}^{\lambda}=\inf \left\{t_{i}>0: X_{t_{i}}<a\right\}
$$

Under Parisian observation times, first passage is recorded only when the most recent excursion below $a$ /above $b$ has exceeded an exponential random variable $e_{\lambda}$ of rate $\lambda$. We use here the same notation as for classic first passage times (which correspond to the case $\lambda=\infty$ ).

Remark 8.1. We will refer to stopping at $T_{0,-}$ as (exponential) Parisian absorption. A spectrally negative Lévy processes with (exponential) Parisian reflection below 0 may be defined by pushing the process up to 0 each time it is below 0 at an observation time $t_{i}$. In both cases, this will not be made explicit in the notation; classic and Parisian absorption and reflection will be denoted in the same way.

Note that the case $\lambda \rightarrow 0$ corresponds to complete leniency; default is never observed. We see thus that Parisian inspection is an intermediate situation between continuous inspection and no inspection, and can help to render modelling more realistic.

\footnotetext{
${ }^{22}$ The concept of periodic observation may be extended to the Sparre Andersen (non Lévy) case, using geometrically distributed intervention times at the times of claims. This deserves further investigation.
} 
It was recently observed that the classic first passage laws listed above hold with a "Parisianly observed" lower boundary, once $W_{q}, Z_{q}$ are replaced by appropriate generalizations, defined by [20, 33]:

$$
\begin{aligned}
& Z_{q, \lambda}(x, \theta):=\frac{\lambda}{q+\lambda-\kappa(\theta)} Z_{q}(x, \theta)+\frac{q-\kappa(\theta)}{q+\lambda-\kappa(\theta)} Z_{q}(x, \Phi(q+\lambda)) \\
& =\frac{\lambda}{q+\lambda-\kappa(\theta)}\left(Z_{q}(x, \theta)-Z_{q}(x, \Phi(q+\lambda))\right)+Z_{q}(x, \Phi(q+\lambda)), \\
& W_{q, \lambda}(x):=\frac{\Phi(q+\lambda)-\Phi_{q}}{\lambda} Z_{q}(x, \Phi(q+\lambda)),
\end{aligned}
$$

with the value for $\theta=\Phi(q+\lambda)$ being interpreted in the limiting sense. ${ }^{23}$

Remark 8.2. Exponential Parisian detection below 0 is related to the Laplace transform of the total "occupation time spent in the red"

$$
T^{<0}:=\int_{0}^{\infty} \mathbb{1}_{\left\{X_{t}<0\right\}} \mathrm{d} t
$$

a fundamental risk measure studied by [103, 123, 154].

Indeed, the probability of Parisian ruin not being observed (and of recovering without bailout) when $\kappa^{\prime}\left(0_{+}\right)>$ 0 is ([106], Cor. 1,Thm. 1; [12], Eq. (11))

$$
P_{x}\left[T_{0,-}=\infty\right]=P_{x}\left[T^{<0}<e_{\lambda}\right]=\mathbb{E}_{x}\left[e^{-\lambda T^{<0}}\right]=\kappa^{\prime}\left(0_{+}\right) \frac{\Phi(\lambda)}{\lambda} Z(x, \Phi(\lambda))
$$

When $x=0$, this reduces to

$$
P_{0}\left[T_{0,-}=\infty\right]=P_{0}\left[T^{<0}<e_{\lambda}\right]=\mathbb{E}_{0}\left[e^{-\lambda T^{<0}}\right]=\kappa^{\prime}\left(0_{+}\right) \frac{\Phi(\lambda)}{\lambda}
$$

a quantity which could be viewed as a model dependent extension of the profit parameter $\kappa^{\prime}\left(0_{+}\right)$, measuring the profitability of a risk process.

Note that $\kappa^{\prime}\left(0_{+}\right) \frac{\Phi(\lambda)}{\lambda}$ furnishes also the Laplace transform of six other remarkable random variables besides $T^{<0}$, by the "Sparre-Andersen identities" due to Proposition 1.1, (2) in [82]. Differentiating (8.5) with respect to $\lambda$ when $\kappa^{\prime}\left(0_{+}\right)>0$ shows that the Sparre-Andersen-Ivanovs variables have all expectation $-\frac{\Phi^{\prime \prime}(0)}{2}=\frac{\kappa^{\prime \prime}(0)}{\left(\kappa^{\prime}(0)\right)^{3}}$, a quantity which appeared already in several previous computations.

The following proposition lists some basic first passage results for processes with Parisian detection of ruin, reflected or absorbed, following $[12,33,40]$. Note that these results coincide with the ones with classic, "hard" detection of ruin, and imply them when $\lambda \rightarrow \infty$.

Theorem 8.3. First passage results for processes with classic detection at a smooth boundary $b>0$ and Parisian detection below 0, followed by stopping or by reflection. Let $X$ be a spectrally negative Lévy process with Parisian detection below 0 , and fix $b>0$. Assuming $x \in[0, b]$ and $q, \lambda>0,0 \leq \theta<\infty$, using the notation of Remark 3.7 and letting $W_{q, \lambda}(x)$ and $Z_{q, \lambda}(x, \theta)$ be defined by (8.2), the following hold:

\footnotetext{
${ }^{23}$ When $\lambda \rightarrow \infty$, the Parisian results reduce to the classic ones, since $Z_{q, \lambda}(x, \theta), W_{q, \lambda}(x)$ are asymptotically equivalent to $Z_{q}(x, \theta), W_{q}(x)$. The first assertion is trivial, for the second see (13.4). The notation $W_{q, \lambda}(x):=\frac{\Phi(q+\lambda)-\Phi_{q}}{\lambda} Z_{q}(x, \Phi(q+\lambda))$ has been chosen to emphasize that this replaces, for processes with Parisian ruin, the $W_{q}$ scale function in the classic "gambler's winning" problem, and also to ensure a convenient asymptotic behavior.
} 
1. The "gamblers survival formula takes the form ([12], Eq. (12))

$$
\mathbb{E}_{x}^{\{0}\left[e^{-q T_{b,+}} \mathbb{1}_{\left\{T_{b,+}<T_{0,-}\right\}}\right]=\frac{W_{q, \lambda}(x)}{W_{q, \lambda}(b)}
$$

2. A) The expected discounted dividends (upper regulation at b) until $T_{0}^{b]}$ are ([12], Eq. (27)):

$$
V^{\vdots 0, b]}(x)=\mathbb{E}_{x}^{b]}\left[\int_{0}^{T_{0,-}} e^{-q t} d U_{t}\right]=\frac{W_{q, \lambda}(x)}{W_{q, \lambda}^{\prime}(b)}=\frac{Z_{q}(x, \Phi(q+\lambda))}{Z_{q}^{\prime}(b, \Phi(q+\lambda))}
$$

B) The expected discounted dividends with reflection at 0 at Parisian times, until the total bail-outs surpass an exponential variable $e_{\theta}([4]$, Eq. (15)) are

$$
V_{U}^{\{0, b]}(x, \theta)=\mathbb{E}_{x}^{\{0, b]}\left[\int_{0}^{\infty} e^{-q s} \mathbb{1}_{\left\{L(s)<e_{\theta}\right\}} \mathrm{d} U(s)\right]=\frac{Z_{q, \lambda}(x, \theta)}{Z_{q, \lambda}^{\prime}(b, \theta)}
$$

Remark 8.4. When $\theta=0$, this becomes ([33], Cor. 3.3):

$$
V_{U}^{\{0, b]}(x)=\mathbb{E}_{x}^{\{0, b]}\left[\int_{0}^{\infty} e^{-q t} \mathrm{~d} U_{t}\right]=\frac{Z_{q, \lambda}(x)}{Z_{q, \lambda}^{\prime}(b)} .
$$

3. The capital injections/bailouts law for a process with Parisian reflection at 0 , until $T_{b,+}$ ([33], Cor. 3.1 ii)). Let $L_{t}$ denote the regulator for the process with Parisian reflection at 0 and $\mathbb{E}_{x}^{\{0}$ the expectation for such process. Then:

$$
\bar{\Psi}_{q, \theta, \lambda}^{\{0, b \mid}(x):=\mathbb{E}_{x}^{\{0}\left[e^{\left.-q T_{b,+}-\theta L_{T_{b,+}}\right]}=\left\{\begin{array}{ll}
\frac{Z_{q, \lambda}(x, \theta)}{Z_{q, \lambda}(b, \theta)} & \theta<\infty \\
\mathbb{E}_{x}^{\{0}\left[e^{-q T_{b,+}} ; T_{b,+}<T_{0,-}\right]=\frac{W_{q, \lambda}(x)}{W_{q, \lambda}(b)} & \theta=\infty
\end{array} .\right.\right.
$$

4. Deficit at ruin for a process absorbed or reflected at $b>0$.

A) The joint Laplace transform of the Parisian first passage time of 0 and the undershoot for a process absorbed at $T_{b,+}$ is given by ([12], Eq. (15)): ${ }^{24}$

$$
\begin{aligned}
\Psi_{q, \theta, \lambda}^{\vdots 0, b \mid}(x):= & \mathbb{E}_{x}\left[e^{\theta X_{T_{0,-}}} \mathbb{1}_{\left\{T_{0,-}<T_{b,+} \wedge e_{q}\right\}}\right]=Z_{q, \lambda}(x, \theta)-W_{q, \lambda}(x) W_{q, \lambda}(b)^{-1} Z_{q, \lambda}(b, \theta) \\
& =\frac{\lambda}{q+\lambda-\kappa(\theta)}\left(Z_{q}(x, \theta)-W_{q, \lambda}(x) W_{q, \lambda}(b)^{-1} Z_{q}(b, \theta)\right)
\end{aligned}
$$

B) The joint Laplace transform of the first passage time at 0 and the undershoot in the presence of reflection at a barrier $b \geq 0$ is

$$
\Psi_{q, \theta, \lambda}^{\vdots: b, b]}(x):=\mathbb{E}_{x}^{b]}\left[e^{-q T_{0}^{b]}+\theta X_{T_{0}^{b]}}}\right]=Z_{q, \lambda}(x, \theta)-\frac{W_{q, \lambda}(x)}{W_{q, \lambda}^{\prime}(b)} Z_{q, \lambda}^{\prime}(b, \theta), x \geq 0 .
$$

\footnotetext{
${ }^{24}$ The second expression in (8.11) uses a simpler, non-smooth Gerber-Shiu function - see Remark (6.16).
} 
5. Let $U_{q, \lambda}^{\vdots a, b \mid}(x, B)=\mathbb{E}_{x}\left[\int_{0}^{T_{a,-} \wedge T_{b,+}} e^{-q t} \mathbb{1}_{\left\{X_{t} \in B\right\}} \mathrm{d} t\right]$, denote the $q$-resolvent of a doubly absorbed spectrally negative Lévy process with Parisian ruin, for any Borel set $B \subset[a, b]$. Then ([40], Thm. 2)

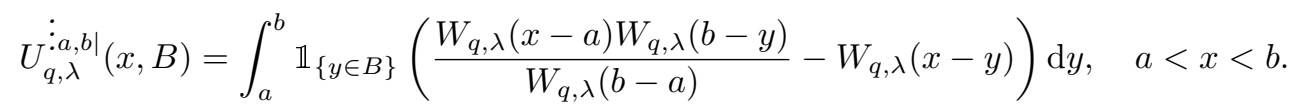

6. The dividends-penalty law for a process reflected at b, with Parisian ruin is:

$$
\begin{gathered}
D P_{q, \theta, \vartheta}^{\vdots 0, b]}(x):=\mathbb{E}_{x}^{b]}\left[e^{-\vartheta U_{T_{0,-}}+\theta X_{T_{0,-}}} ; T_{0,-}<e_{q}\right]=Z_{q, \lambda}(b, \theta)-W_{q, \lambda}(b) \frac{Z_{q, \lambda}^{\prime}(b, \theta)+\vartheta Z_{q, \lambda}(b, \theta)}{W_{q, \lambda}^{\prime}(b)+\vartheta W_{q, \lambda}(b)} \\
=\left(Z_{q}(x, \theta)-Z_{q, \Phi(q+\lambda)}(x) H_{\Phi(q+\lambda)}(b)^{-1} H_{\theta}(b)\right) \lambda(q+\lambda-\kappa(\theta))^{-1},
\end{gathered}
$$

where $H_{\theta}(b)=\vartheta Z_{q}(b, \theta)+Z_{q}^{\prime}(b, \theta)=(\theta+\vartheta) Z_{q}(b, \theta)+(q-\kappa(\theta)) W_{q}(b) .{ }^{25}$ We included the second, rather complicated formula, to allow comparison with the original formula in (23) from [12].

Remark 8.5. When $x=b$, we may factorize the transform $\mathbb{E}_{b}^{b]}\left[e^{\theta X_{T_{0}}-\vartheta U_{T_{0}}} ; T_{0}<e_{q}\right]$ (8.15) as:

$$
\frac{\nu_{q, \lambda}}{\nu_{q, \lambda}+\vartheta}\left(Z_{q}(b, \theta)-\nu_{q, \lambda}{ }^{-1}\left(\theta Z_{q}(b, \theta)+(q-\kappa(\theta)) W_{q}(b)\right)\right) \frac{\lambda}{\lambda+q-\kappa(\theta)},
$$

where $\nu_{q, \lambda}=V^{b]}(b)^{-1}=W_{q, \lambda}^{\prime}(b) W_{q, \lambda}(b)^{-1}=Z_{q, \Phi_{q+\lambda}}^{\prime}(b) Z_{q, \Phi_{q+\lambda}}(b)^{-1}$. Indeed,

$$
\begin{aligned}
& Z_{q}(b, \theta)-Z_{q, \Phi_{q+\lambda}}(b)\left(\left(\Phi_{q+\lambda}+\vartheta\right) Z_{q, \Phi_{q+\lambda}}(b)-\lambda W_{q}(b)\right)^{-1} H_{\theta}(b) \\
& =Z_{q}(b, \theta)-\left(\vartheta+\Phi_{q+\lambda}-\lambda W_{q}(b) Z_{q, \Phi_{q+\lambda}}(b)^{-1}\right)^{-1} H_{\theta}(b) \\
& =Z_{q}(b, \theta)-\left(\vartheta+\nu_{q, \lambda}\right)^{-1} H_{\theta}(b),
\end{aligned}
$$

and (8.16) follows by simple algebra. By (8.16), $U_{T_{0}}$ and $X_{T_{0}}$ are independent when starting from $b$, and the former has an exponential distribution with parameter $\nu_{q, \lambda}([12],(23),(26))$.

When $\vartheta=0$, this result reduces to (8.12).

7. A) The expected total discounted bailouts at Parisian times up to $T_{b,+}$ are given for $0 \leq x \leq b$ and $q>0$ by ([33], Cor. 3.2 ii)):

$$
B^{\{0, b \mid}(x):=\mathbb{E}_{x}^{[0}\left[\int_{0}^{T_{b,+}} e^{-q t} \mathrm{~d} L_{t}\right]=\frac{Z_{q, \lambda}(x)}{Z_{q, \lambda}(b)} G_{q, \lambda}^{B}(b)-G_{q, \lambda}^{B}(x)
$$

where

$$
G_{q, \lambda}^{B}(x)=\frac{\lambda}{q+\lambda}\left(\bar{Z}_{q}(x)+\frac{\kappa^{\prime}\left(0_{+}\right)}{q}\right)=\frac{\lambda}{q+\lambda} G_{q}^{B}(x) .
$$

\footnotetext{
${ }^{25}$ The structure of this formula reflects the fact that $\Phi(q+\lambda)$ is a removable singularity.
} 
B) The expected total discounted bailouts at Parisian times over an infinite horizon, with reflection at $b$ are ([33], Cor. 3.4) (see also Thm. 3.2 from [152], where $Z_{q, \lambda}(x)$ is denoted by $\left.B_{2}(x)^{26}\right)$ :

$$
V^{\{0, b]}(x)=\mathbb{E}_{x}^{[0, b]}\left[\int_{0}^{\infty} e^{-q t} \mathrm{~d} L_{t}\right]=\frac{Z_{q, \lambda}(x)}{Z_{q, \lambda}^{\prime}(b)}\left(G_{q, \lambda}^{B}\right)^{\prime}(b)-G_{q, \lambda}^{B}(x) .
$$

Remark 8.6. Note that each result from Theorem 8.3 has its analog in classical detection of ruin. Indeed,

- (1) corresponds to the dividends Theorem 6.1;

- (2) is the Parisian analog of the bail-outs Theorem 6.3 ([78], Thm. 2);

- (3) A) and B) are Parisian analogues of Theorem 6.5 A) and B) ([32]), Prop. 5.5);

- (4) corresponds to the resolvent formula (3.16); it is natural to conjecture that the resolvents for (partly) reflected processes will also be of the same form as the classic ones ([124], Thm. 1; [81], Thm. 2, Cor. 2);

- (5) is the Parisian analog of the dividends-penalty Theorem 6.8;

- (6) corresponds to the expected total discounted bailouts Theorem 6.15. One may check that

$$
V_{q, \theta, \lambda}^{\{0, b]}(x)=\mathbb{E}_{x}^{[0, b]}\left[\int_{0}^{\infty} e^{-q t} \mathbb{1}_{\left\{L(s)<e_{\theta}\right\}} \mathrm{d} L_{t}\right]=\frac{Z_{q, \lambda}(x, \theta)}{Z_{q, \lambda}^{\prime}(b, \theta)}\left(G_{q, \lambda}^{B}\right)^{\prime}(b)-G_{q, \lambda}^{B}(x) .
$$

Problem 8.7. It is natural to conjecture that the outstanding results which have not yet been extended from the classic to the Parisian case, like Theorem 6.13 on the joint distribution of dividends and bailouts, the optimality of barrier policies with fixed final penalty (9.11) ([75], Prop. 4.3), the optimality of barrier policies for the Shreve, Lehoczky and Gaver objective ([31], Lem. 2) etc., hold in the Parisian case as well.

Problem 8.8. The fact that the results for the Parisian case coincide with the classical ones suggest that the known first passage results with hard ruin for SNMAPs [3, 78, 79, 89] might generalize to the Parisian case, provided that properly defined scale matrix functions are introduced, and multiplied in correct order. To facilitate further work, we provide non-Parisian SNMAP references for the corresponding results of Theorem 8.3: for (2) A) and B) see Corollary 3 in [78] and Theorem 6 in [78] respectively; for (3) see Theorem 2 in [78]; for (4) see Theorem 2 and Corollary 2 in [81]; for (5) see Theorem 6 in [78].

Most interesting is the problem of resolvents. One case already resolved is the resolvent density $u_{q, \lambda}^{\{0}(x, y)$ with Parisian reflection at Poisson observation times of intensity $\lambda$, obtained in Theorem 4.1 in [132]. It is not easy to prove that their result converges when $\lambda \rightarrow \infty$ to the classic one in (22), Corollary 2 in [81].

Problem 8.9. It would be interesting to generalize the $W, Z$ formalism in a way which applies also to the case of periodic observations of the smooth boundary.

Remark 8.10. Some of the results above have been extended to processes $X_{\delta}^{[0[}(t)$ with classic reflection at 0 and refraction at the maximum ([4], Eq. (3),Thm. 3.1), and to processes $X_{\delta}^{b[}(t)$ with $\delta$-refraction at a fixed point $b[86,90,94,131,134]$.

Thus, (8.10) holds with $Z_{q}(x, \theta)$ replaced by $Z_{q}^{\frac{1}{1-\delta}}(x, \theta)$ ([4], Thm. 3.1). The proof uses the probabilistic interpretation $\mathbb{E}_{x}^{[0}\left[e^{\left.-q T_{b,+}-\theta L_{T_{b,+}}\right]}=P\left[T_{b,+}<e_{q} \wedge K_{\theta}\right]\right.$, where $K_{\theta}$ is the first time when the total bail-out exceeds an independent exponential random variable $e_{\theta}$. Finally, (22) in [12] extend this to the case when $T_{b,+}$ is replaced by its Parisian version.

\footnotetext{
${ }^{26}$ Our sign of $\frac{p}{q}$ in formula (8.18) for $G_{B}(x)$ is opposite to that in formulas (3.26) and (3.30) of [152], since they consider spectrally positive processes.
} 
Similar results hold also for processes $X_{q}^{b[}(t)$ with $\delta$-refraction at a fixed point $b[86,90,131,134]$. The scale functions are:

$$
\begin{gathered}
w_{q}^{b[}(x)=W_{q}(x)+\delta \int_{b}^{x} \mathbb{W}_{q}(x-y) W_{q}^{\prime}(y) \mathrm{d} y, \\
z_{q}^{b[}(x, \theta)=Z_{q}(x, \theta)+\delta \int_{b}^{x} \mathbb{W}_{q}(x-y) Z_{q}^{\prime}(y, \theta) \mathrm{d} y,
\end{gathered}
$$

where $\mathbb{W}_{q}$ is the scale function of $X_{t}-\delta t$.

For example, by Corollary 2 in [90], it holds that

$$
\mathbb{E}_{x}\left[e^{-\lambda T^{<0}}\right]=P_{x}\left[T_{0,-}=\infty\right]=\left(\kappa^{\prime}\left(0_{+}\right)-q\right) \frac{\Phi(\lambda)}{\lambda-q \Phi(\lambda)} z_{q}^{b[}(x, \Phi(\lambda)), \quad 0 \leq q \leq \kappa^{\prime}\left(0_{+}\right) .
$$

\subsection{Elements of proof for Theorem 8.3}

In the following, we provide some proofs for Theorem 8.3. Before that, let us record some useful preliminaries.

Proposition 8.11. For $z \leq 0$, it holds that

A) the "recovery before Parisian ruin" probability is

$$
\begin{aligned}
& P_{z}\left[T_{\{0\}}<e_{\lambda}\right]=\mathbb{E}\left[e^{-\lambda T_{\{0\}}}\right]=e^{\Phi(\lambda) z} \\
& \mathbb{E}_{z}\left[e^{-q T_{\{0\}}} ; T_{\{0\}}<e_{\lambda}\right]=\mathbb{E}\left[e^{-(\lambda+q) T_{\{0\}}}\right]=e^{\Phi(\lambda+q) z}
\end{aligned}
$$

B)

$$
\mathbb{E}_{z}\left[e^{-q e_{\lambda}+\theta X_{e_{\lambda}}} ; T_{\{0\}}<e_{\lambda}\right]=e^{\Phi(q+\lambda) z} \mathbb{E}_{0}\left[e^{-q e_{\lambda}+\theta X_{e_{\lambda}}}\right]=e^{\Phi(q+\lambda) z} \frac{\lambda}{\lambda+q-\kappa(\theta)}, \forall \theta \neq \Phi(\lambda)
$$

C)

$$
\mathbb{E}_{z}\left[e^{-q e_{\lambda}+\theta X_{e_{\lambda}}} ; e_{\lambda}<T_{\{0\}}\right]=\mathbb{E}_{z}\left[e^{-q e_{\lambda}+\theta X_{e_{\lambda}}}\right]-e^{\Phi(\lambda) z} \mathbb{E}\left[e^{\theta X_{e_{\lambda}}}\right]=\frac{\lambda}{\lambda+q-\kappa(\theta)}\left(e^{\theta z}-e^{\Phi(\lambda+q) z}\right), \quad \theta \geq 0 .
$$

Proof. A) The second equation follows from the first, which is just the fundamental identity (3.3) (or set $z \leq 0$ in $(6.14))$. B) follows by the strong Markov property at $T_{\{0\}}$, and C) follows from B).

Proof of Theorem 8.3 2. By the strong Markov property, we may decompose $l(x, b):=\mathbb{E}_{x}\left[e^{-q T_{b,+}-\theta L_{T_{b,+}}}\right], \theta>$ $\lambda+q$, in three parts:

$$
\begin{aligned}
l(x, b)= & \mathbb{E}_{x}\left[e^{-q T_{b,+}} ; T_{b,+}<T_{0}\right]+\mathbb{E}_{x}\left[e^{-q T_{0}} \mathbb{E}_{X_{T_{0}}}\left[e^{-q T_{\{0\}}} ; T_{\{0\}}<e_{\lambda}\right] ; T_{0}<T_{b,+}\right] l(0, b) \\
& +\mathbb{E}_{x}\left[e^{-q T_{0}} \mathbb{E}_{X_{T_{0}}}\left[e^{-q e_{\lambda}+\theta X_{e_{\lambda}}} ; e_{\lambda}<T_{\{0\}}\right] ; T_{0}<T_{b,+}\right] l(0, b)=\frac{W_{q}(x)}{W_{q}(b)} \\
+l(0, b)[ & \left.\mathbb{E}_{x}\left[e^{-q T_{0}+\Phi(q+\lambda) X_{T_{0}}} ; T_{0}<T_{b,+}\right]+C\right]=\frac{W_{q}(x)}{W_{q}(b)}+l(0, b)\left[Z_{q}(x, \Phi(q+\lambda))-W_{q}(x) \frac{Z_{q}(b, \Phi(q+\lambda))}{W_{q}(b)}+C\right]
\end{aligned}
$$

where we have used Proposition 8.11 A). 
For the third part we use Proposition 8.11 C). We find

$$
\begin{aligned}
& C=\mathbb{E}_{x}\left[e^{-q T_{0}} \mathbb{E}_{X_{T_{0}}}\left[e^{-q e_{\lambda}+\theta X_{e_{\lambda}}} ; e_{\lambda}<T_{\{0\}}\right] ; T_{0}<T_{b,+}\right] \\
& =\frac{\lambda}{\lambda+q-\kappa(\theta)} \mathbb{E}_{x}\left[e^{-q T_{0}}\left(e^{\theta X_{T_{0}}}-e^{\Phi(\lambda+q) X_{T_{0}}}\right) ; T_{0}<T_{b,+}\right] .
\end{aligned}
$$

Finally

$$
\begin{aligned}
l(x, b)= & \left\{\frac{\lambda}{\lambda+q-\kappa(\theta)}\left(Z_{q}(x, \theta)-Z_{q}(x, \Phi(q+\lambda))-W_{q}(x) \frac{Z_{q}(b, \theta)-Z_{q}(b, \Phi(q+\lambda))}{W_{q}(b)}\right)+Z_{q}(x, \Phi(q+\lambda))\right. \\
& \left.-W_{q}(x) \frac{Z_{q}(b, \Phi(q+\lambda))}{W_{q}(b)}\right\} l(0, b)+\frac{W_{q}(x)}{W_{q}(b)}=\left\{Z_{q, \lambda}(x, \theta)-W_{q}(x) \frac{Z_{q, \lambda}(b, \theta)}{W_{q}(b)}\right\} l(0, b)+\frac{W_{q}(x)}{W_{q}(b)} .
\end{aligned}
$$

Now in the finite variation case we may substitute $x=0$, and, using $W_{q}(0)>0$, conclude that $l(0, b)=$ $\frac{1}{Z_{q, \lambda}(b, \theta)}$, which yields the result.

In the infinite variation case, we may use a perturbation approach. For $b>x>0$, we have

$$
\begin{aligned}
l(0, b)=\mathbb{E}\left[e^{-q \tau_{x}^{+}} ; \tau_{x}^{+}<e_{\lambda}\right] l(x, b)+\mathbb{E}\left[e^{-q e_{\lambda}+\theta X_{e_{\lambda}}} ; e_{\lambda}<\tau_{x}^{+}, X_{e_{\lambda}}<0\right] l(0, b) \\
+\int_{0}^{x} \mathbb{E}\left[e^{-q e_{\lambda}} ; e_{\lambda}<\tau_{x}^{+}, X_{e_{\lambda}} \in \mathrm{d} y\right] l(y, b) \mathrm{d} y=e^{-\Phi(q+\lambda) x} l(x, b)+I_{2}(x) l(0, b)+I_{3}(x), \\
I_{2}(x)=\lambda \int_{-\infty}^{0}\left(e^{-\Phi(q+\lambda) x} W_{\lambda+q}(x-y)-W_{\lambda+q}(-y)\right) e^{\theta y} \mathrm{~d} y \\
=\lambda \int_{0}^{\infty} e^{-\Phi(q+\lambda) x-\theta y} W_{\lambda+q}(x+y) \mathrm{d} y-\frac{\lambda}{\kappa(\theta)-q-\lambda} \\
=\lambda \int_{x}^{\infty} e^{-\Phi(q+\lambda) x-\theta(z-x)} W_{q+\lambda}(z) \mathrm{d} z-\frac{\lambda}{\kappa(\theta)-q-\lambda} \\
=\frac{\lambda}{\kappa(\theta)-q-\lambda}\left(e^{-\Phi(q+\lambda) x+\theta x}-1\right)-\lambda \int_{0}^{x} e^{-\Phi(q+\lambda) x-\theta(z-x)} W_{q+\lambda}(z) \mathrm{d} z \\
=\frac{\lambda}{\kappa(\theta)-q-\lambda}\left(e^{-\Phi(q+\lambda) x+\theta x}-1\right)+o\left(W_{q}(x)\right) .
\end{aligned}
$$

We can check that

$$
\begin{aligned}
& e^{-\Phi(q+\lambda) x}\left(Z_{q}(x, \Phi(q+\lambda))-Z_{q}(x, \theta)\right) \\
& =e^{-\Phi(q+\lambda) x}\left[e^{\Phi(q+\lambda) x}\left(1-\lambda \int_{0}^{x} e^{-\Phi(q+\lambda) y} W_{r}(y) \mathrm{d} y\right)-e^{\theta x}\left(1-\lambda \int_{0}^{x} e^{-\theta y} W_{r}(y) \mathrm{d} y\right)\right] \\
& =1-e^{-\Phi(q+\lambda) x+\theta x}+o\left(W_{q}(x)\right), \\
& Z_{q}(x, \Phi(q+\lambda))=e^{\Phi(q+\lambda) x}\left(1-q \int_{0}^{x} e^{-\Phi(q+\lambda) y} W_{q}(y) \mathrm{d} y\right)=e^{\Phi(q+\lambda) x}+o\left(W_{q}(x)\right), \text { and } \\
& I_{3}(x) \leq \int_{0}^{x} E\left[e^{-q e_{\lambda}} ; e_{\lambda}<\tau_{x}^{+}, X_{e_{\lambda}} \in \mathrm{d} y\right] \mathrm{d} y=\lambda \int_{0}^{x} e^{-\Phi(q+\lambda) x} W_{q+\lambda}(x-y) \mathrm{d} y=o\left(W_{q}(x)\right) .
\end{aligned}
$$


Solving now (8.24) for $l(0, b)$ and letting $x \rightarrow 0+$, we find again

$$
\begin{aligned}
l(0, b) & =\lim _{x \rightarrow 0+} \frac{e^{-\Phi(q+\lambda) x} \frac{W_{q}(x)}{W_{q}(b)}}{e^{-\Phi(q+\lambda) x} W_{q}(x) \frac{Z_{q}(b, \Phi(q+\lambda))}{W_{q}(b)}+\lambda e^{-\Phi(q+\lambda) x} W_{q}(x) \frac{Z_{q}(b, \Phi(q+\lambda))-Z_{q}(b, \theta)}{(\kappa(\theta)-q-\lambda) W_{q}(b)}+o\left(W_{q}(x)\right)} \\
& =\frac{\kappa(\theta)-q-\lambda}{(\kappa(\theta)-q) Z_{q}(b, \Phi(q+\lambda))-\lambda Z_{q}(b, \theta)}=\frac{1}{Z_{q, \lambda}(b, \theta)} .
\end{aligned}
$$

\subsection{Spectrally negative Omega pocesses}

Recently, it was discovered that the classic exponential Parisian formulas may be further extended to Omega models, $[10,74,104,114]$, in which a state-dependent rate of killing (or observation) rate $\omega(x)$ is used, where $\omega: \mathbb{R} \rightarrow \mathbb{R}_{+}$is an arbitrary locally bounded nonnegative measurable function. Exponential Parisian models are just the particular case when $\omega(x)$ is a step function with two values.

Analogs of Propositions 3.2, 3.8 and of Theorems 6.5, 6.3 are provided in Theorems 2.1-2.4 in [104], who showed that the first passage theory of Omega models rests on two functions $\left\{\mathcal{W}_{\omega}(x), x \in \mathbb{R}\right\}$ and $\left\{\mathcal{Z}_{\omega}(x), x \in \mathbb{R}\right\}$ called $\omega$-scale functions, which are defined uniquely as the solutions of the renewal equations:

$$
\begin{aligned}
& \mathcal{W}_{\omega}(x)=W(x)+\int_{0}^{x} W(x-y) \omega(y) \mathcal{W}_{\omega}(y) \mathrm{d} y \\
& \mathcal{Z}_{\omega}(x)=1+\int_{0}^{x} W(x-y) \omega(y) \mathcal{Z}_{\omega}(y) \mathrm{d} y
\end{aligned}
$$

where $W(x)$ is the classical zero scale function.

Furthermore, (8.25), (8.26) may be generalized to nonhomogeneous models ([114], Lem. 3):

$$
\begin{aligned}
& \mathcal{W}_{\widetilde{\omega}}(x, a)=\mathcal{W}_{\omega}(x, a)+\int_{0}^{x} \mathcal{W}_{\omega}(x, y)(\widetilde{\omega}(y)-\omega(y)) \mathcal{W}_{\widetilde{\omega}}(y, a) \mathrm{d} y \\
& \mathcal{Z}_{\widetilde{\omega}}(x, a)=Z_{\omega}(x, a)+\int_{0}^{x} W_{\omega}(x, y)(\widetilde{\omega}(y)-\omega(y)) \mathcal{Z}_{\widetilde{\omega}}(y, a) \mathrm{d} y
\end{aligned}
$$

Note that in the case of constant $\omega(x)=q$, these reduce

$$
W_{q}-W=q W_{q} * W \quad \text { and } \quad Z_{q}-Z=q W_{q} * Z,
$$

which can be easily checked by taking the Laplace transforms of their both sides and by using the expansion (6.30).

\subsection{Occupation times}

Here is an elegant result ([115], Thm. 3.1) on the joint law of the occupation times above and below 0 of a spectrally negative Lévy process.

Proposition 8.12. Introduce the auxiliary function ([115], (1)) (a slight modification of which had essentially appeared already in (6) from [108]), defined for all $x \in \mathbb{R}$ and $\lambda, q \geq 0$ by:

$$
\mathcal{W}_{\lambda, q}^{a}(x):= \begin{cases}W_{\lambda}(x), & 0 \leq x \leq a \\ W_{\lambda}(x)+(q-\lambda) \int_{a}^{x} W_{q}(x-y) W_{\lambda}(y) \mathrm{d} y=W_{q}(x)+(\lambda-q) \int_{0}^{a} W_{q}(x-y) W_{\lambda}(y) \mathrm{d} y, & 0 \leq a \leq x \\ W_{q}(x), & a \leq 0\end{cases}
$$


where the second equalities hold by the convolution identity $W_{\lambda} * W_{q}(x)=\frac{W_{\lambda}(x)-W_{q}(x)}{\lambda-q}([108](5)) .{ }^{27}$ Let $L_{t}^{-}=$ $\int_{0}^{t} \mathbb{1}_{(-\infty, 0)}\left(X_{s}\right) \mathrm{d} s, L_{t}^{+}=\int_{0}^{t} \mathbb{1}_{(0, \infty)}\left(X_{s}\right) \mathrm{d} s$ denote the occupation times below and above 0 . Then, $\forall \lambda_{-}, \lambda_{+}>0$ and $\forall x, y \in \mathbb{R}$ it holds that

$$
\begin{aligned}
& \int_{0}^{\infty} e^{-q t} \mathbb{E}_{x}\left[e^{-\lambda_{-} L_{t}^{-}-\lambda_{+} L_{t}^{+}}, X_{t} \in \mathrm{d} y\right] \mathrm{d} t \\
& =\left(\frac{\Phi\left(q+\lambda_{+}\right)-\Phi\left(q+\lambda_{-}\right)}{\lambda_{+}-\lambda_{-}} Z_{q+\lambda_{+}}\left(x, \Phi\left(q+\lambda_{-}\right)\right) Z_{q+\lambda_{-}}\left(-y, \Phi\left(q+\lambda_{+}\right)\right)-\mathcal{W}_{q+\lambda_{-}, q+\lambda_{+}}^{-y}(x-y)\right) \mathrm{d} y .
\end{aligned}
$$

Remark 8.13. Starting from $x=0$, the result loses its symmetry, and simplifies to ([115], Thm. 3.1, Rem. 3.2)

$$
\begin{aligned}
(\mathrm{d} y)^{-1} \int_{0}^{\infty} e^{-q t} \mathbb{E}_{0}\left[e^{-\lambda_{-} L_{t}^{-}-\lambda_{+} L_{t}^{+}}, X_{t} \in \mathrm{d} y\right] \mathrm{d} t & =\frac{\Phi\left(q+\lambda_{+}\right)-\Phi\left(q+\lambda_{-}\right)}{\lambda_{+}-\lambda_{-}} Z_{q+\lambda_{-}}\left(-y, \Phi\left(q+\lambda_{+}\right)-W_{q+\lambda_{-}}(-y)\right. \\
& =\frac{\Phi\left(q+\lambda_{+}\right)-\Phi\left(q+\lambda_{-}\right)}{\lambda_{+}-\lambda_{-}} \mathbb{E}_{-y}\left[e^{-\left(q+\lambda_{-}\right) T_{0}+\Phi\left(q+\lambda_{+}\right) X_{T_{0}}}\right] .
\end{aligned}
$$

Integrating the final position yields ([115], Cor. 3.1)

$$
\int_{0}^{\infty} e^{-q t} \mathbb{E}_{0}\left[e^{-\lambda_{-} L_{t}^{-}-\lambda_{+} L_{t}^{+}}\right]\left[\text {Cor. 3.1]t }=\frac{\Phi\left(q+\lambda_{-}\right)}{\left(q+\lambda_{-}\right) \Phi\left(q+\lambda_{+}\right)} .\right.
$$

This implies ([106], Rem. 4.1; [138], Cor. 3.2)

$$
\int_{0}^{\infty} e^{-q t} \mathbb{E}_{0}\left[e^{-\lambda L_{t}^{+}}\right] \mathrm{d} t=\frac{\Phi(q)}{q \Phi(q+\lambda)}
$$

Remark 8.14. Asymptotics of occupation times for a reflected process. A general result for the time $L_{t}^{[0, b]}=\int_{0}^{t} \mathbb{1}_{[0, b]}\left(X_{s}\right) \mathrm{d} s$ spent in $[0, b]$ by a process with positive drift (and thus with $\Phi(0)=0$ ) reflected at $b$ is provided in Theorem 3.4 in [138]:

$$
\int_{0}^{\infty} e^{-q t} \mathbb{E}_{0}\left[e^{-\lambda L_{t}^{[0, b]}}\right] \mathrm{d} t=\frac{\Phi(q)}{q} \frac{Z_{\lambda}\left(b, \Phi_{q}\right)}{\lambda W_{\lambda}(b)+\Phi(q) Z_{\lambda}\left(b, \Phi_{q}\right)},
$$

which recovers the previous result (8.31) by using $\lim _{b \rightarrow \infty} \frac{Z_{\lambda}\left(b, \Phi_{q}\right)}{W_{\lambda}(b)}=\frac{\lambda}{\Phi(q+\lambda)-\Phi(q)}$.

The large deviations rate for $L_{t}^{[0, b]}$ has been obtained in Theorem 3.3 in [138], as a direct consequence of the Gärtner-Ellis theorem, which states that this is the Legendre transform of

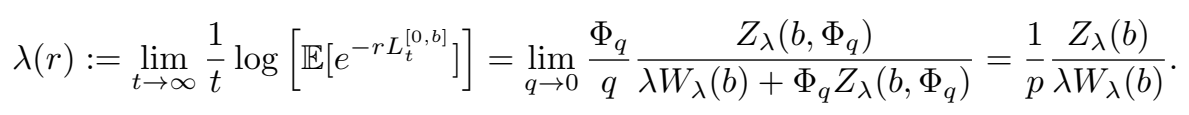

\section{Optimization OF Dividends}

Risk theory initially revolved around minimizing the probability of ruin. However, insurance companies are realistically more interested in maximizing company value than minimizing risk and an alternative approach is therefore to study optimal dividend policies, in the sense of maximizing the expected value of the sum of discounted future dividend payments until the time of ruin, as suggested by De Finetti in the 1950 [56]- see also Miller and Modigliani [117].

\footnotetext{
${ }^{27}$ Note that these functions satisfy $([33],(2.18)) \lim _{a \downarrow-\infty} \frac{\mathcal{W}_{\lambda, q}^{a}(x)}{W_{\lambda}(a)}=Z_{q}(x, \Phi(\lambda)) \quad$ and $\quad \lim _{x \uparrow \infty} \frac{\mathcal{W}_{\lambda, q}^{a}(x)}{W_{q}(x)}=Z_{\lambda}(a, \Phi(q))$.
} 
A second interesting objective to maximize introduced by Shreve, Lehoczky and Gaver (1984) [140], is the expected discounted cumulative dividends for the reflected process obtained by redressing the reserves by capital injections, at a proportional cost, each time this becomes necessary.

These two objectives and certain generalizations are easily expressed for spectrally negative Lévy processes in terms of the scale functions $W, Z$ (at least when restricting to barrier policies).

\subsection{The de Finetti objective with Dickson-Waters modification for spectrally negative processes}

This objective proposed by de Finetti (1957) [56] is to maximize expected discounted dividends until the ruin time. It makes sense to include a penalization for the final deficit [66], arriving at:

$$
\begin{aligned}
& V_{w}(x)=\sup _{\pi} V_{w}^{\pi}(x), \\
& V_{w}^{\pi}(x)=\mathbb{E}_{x}\left[\int_{0}^{T_{0}} e^{-q t} \mathrm{~d} U_{t}^{\pi}+e^{-q T_{0}} w\left(X_{T_{0}}\right)\right]:=V^{\pi}(x)+\Psi_{q, w}^{\pi}(x) .
\end{aligned}
$$

Here $U_{t}^{\pi}$ is an "admissible" dividend paying policy, and $w(x)$ is a bail-out penalty function. ${ }^{28}$

The most important class of policies is that of constant barrier policies $\pi_{b}$, which modify the surplus only when $X_{t}>b$, by a lump payment bringing the surplus at $b$, and then keep it there by Skorokhod reflection, until the next negative jump, ${ }^{29}$ until the next claim.

Under a reflecting barrier strategy $\pi_{b}$, the dividend part of the de Finetti objective has a simple expression (6.1) in terms of the $W$ scale function:

$$
V^{b]}(x)=\mathbb{E}_{x}^{\mid 0, b]}\left[\int_{\left[0, T_{0}^{b]}\right]} e^{-q t} \mathrm{~d} U_{t}\right]=\frac{W_{q}(x)}{W_{q}^{\prime}(b)},
$$

where $\mathbb{E}^{[0, b]}$ denotes the law of the process reflected from above at $b$, and absorbed at 0 and below. This formula reflects the representation

$$
V^{b]}(x)=\mathbb{E}\left[e^{-q T_{b,+}} ; T_{b,+}<T_{0}\right] \mathbb{E}_{b}^{[0, b]}\left[\int_{\left[0, T_{0}^{b]}\right]} e^{-q t} \mathrm{~d} U_{t}\right]=\mathbb{E}\left[e^{-q T_{b,+}} ; T_{b,+}<T_{0}\right] \mathbb{E}_{b}^{[0, b]}\left[U_{T_{0}^{b]} \wedge e_{q}}\right],
$$

and the fact that the local time $U_{t}$ at $b$ with reflection at $b$ is an exponential random variable.

The "barrier function"

$$
H_{D}(b):=\frac{1}{W_{q}^{\prime}(b)}, b \geq 0
$$

plays a central role in the solution of the problem, and the optimal dividend policy is often a barrier strategy at its maximum. In particular, when the barrier function is differentiable and has a unique local maximum $b^{*}>0 \Longrightarrow W_{q}^{\prime \prime}\left(b^{*}\right)=0$, this $b^{*}$ yields the optimal dividend policy. Furthermore, the value function

$$
V(x)=\sup _{b \geq 0} V^{b]}(x)=V^{\left.b^{*}\right]}(x)
$$

\footnotetext{
${ }^{28}$ The value function must satisfy in a viscosity sense the HJB equation $([36],(1.21)): \mathcal{G}(V)(x):=\max \left[\mathcal{G}_{q} V(x), 1-V^{\prime}(x), V(x)-\right.$ $w(x)]=0$, where $\mathcal{G}_{q} V(x)$ denotes the discounted infinitesimal generator of the uncontrolled surplus process, associated to the policy of continuing without paying dividends. The second operator $1-V^{\prime}(x)$ is associated to the possibility of modifying the surplus by a lump payment, and the third to bankruptcy.

${ }^{29}$ In the absence of a Brownian component, this amounts to paying all the income while at $b$.
} 


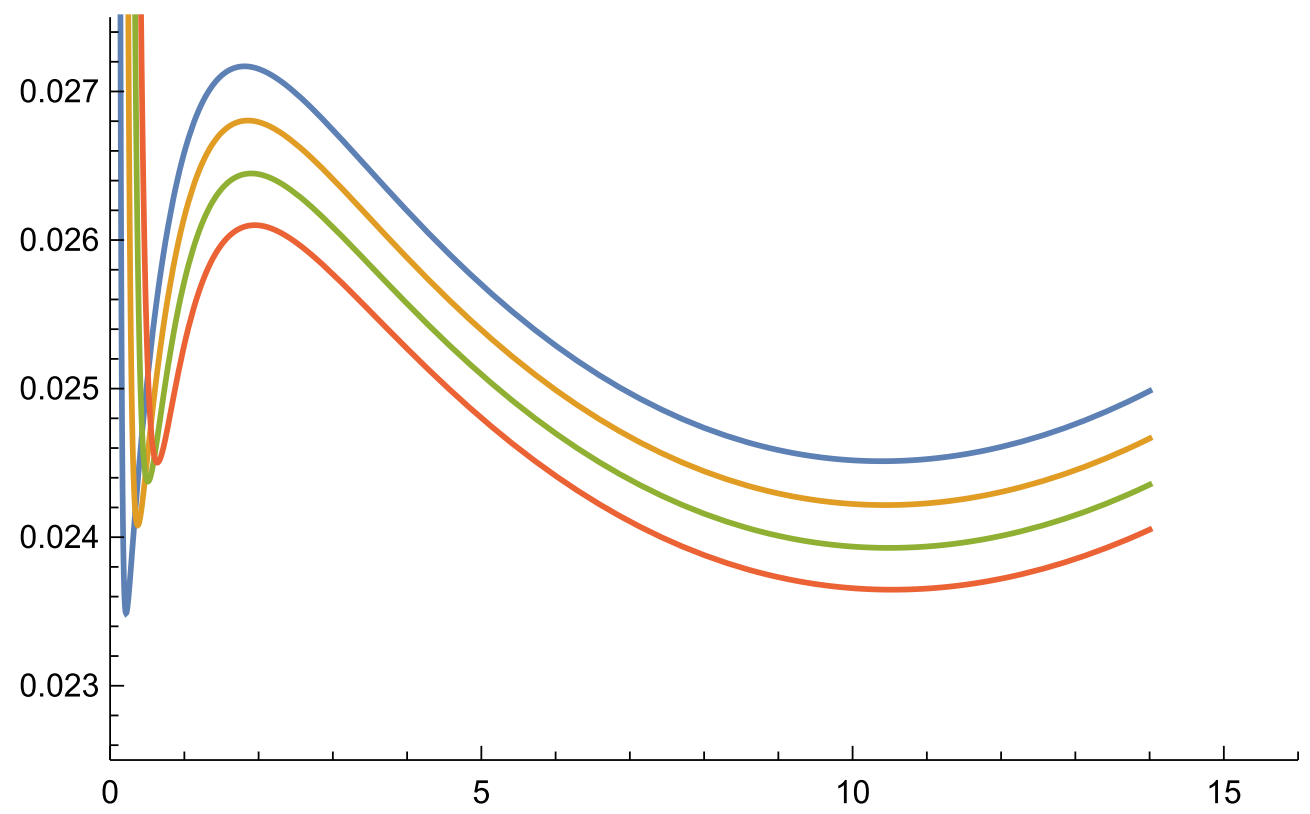

Figure 2. Graphs of the Loeffen example for $\kappa(s)=\frac{\sigma^{2} s^{2}}{2}+c s+\lambda\left(\frac{1}{(s+1)^{2}}-1\right)-q, c=$ $\frac{107}{5}, \lambda=10, q=\frac{1}{10}, \sigma^{2} / 2 \in\{1 / 2,1,3 / 2,2\}$.

is then the largest concave minorant of $W_{q}(x)$. In the presence of several inflection points, however the optimal policy is multiband $[32,35,102,139]$.

The first numerical examples of multiband policies were produced in [35, 102], by Cramér-Lundberg model (1.1) with Erlang claims $E_{2,1}$. However, it was shown in [102] that multibands cannot occur when $W_{q}^{\prime}(x)$ is increasing after its last global minimum $b^{*}$ (i.e. when no local minima are allowed after the global minimum). ${ }^{30}$

[102] further made the interesting observation that in the Brownian perturbed Cramér-Lundberg model (2.5) with Erlang claims $E_{2,1}$ (which are non-monotone), multiband policies may occur for $\sigma$ smaller than a threshold value, but barrier polices (with non-concave value function!) will occur when $\sigma$ is big enough.

Figure 2 displays the first derivative $W_{q}^{\prime}(x)$, for $\sigma^{2} / 2 \in\{1 / 2,1,3 / 2,2\}$. The last two values yield barrier polices with non-concave value function, due to the presence of an inflection point in the interior of the interval $\left[0, b^{*}\right]$.

Even when barrier strategies do not achieve the optimum, and multi-band policies must be used instead, constructing the solution must start by determining the global maximum of the barrier function [32, 35, 139]. We will only consider barrier strategies in this review.

The penalty part of the objective (9.1) for a barrier strategy $\pi_{b}$ can be expressed as $\Psi_{q, w}^{b}(x)=G_{w}(x)-$ $W_{q}(x) \frac{G_{w}^{\prime}(b)}{W_{q}^{\prime}(b)}(7.2)$, where $G_{w}(x)$ is the smooth Gerber-Shiu function associated to the penalty $w$ (see Sect. 7); finally, the modified de Finetti value function is:

$$
V_{w}^{b]}(x)=\left\{\begin{array}{ll}
G_{w}(x)+W_{q}(x) \frac{1-G_{w}^{\prime}(b)}{W_{q}^{\prime}(b)} & x \leq b \\
x-b+V_{w}^{b]}(b) & x \geq b
\end{array} .\right.
$$

\footnotetext{
${ }^{30}$ One instance when that happens is when the Lévy measure is completely monotone. Then, (3.13) may be written as $W_{q}(x)=$ $\Phi_{q}^{\prime} e^{\Phi_{q} x}-\Phi_{q}^{\prime} \int_{0}^{\infty} e^{-x t} \mu_{q}(\mathrm{~d} t), x \geq 0$, for some finite measure $\mu_{q}$. This implies $W_{q}^{\prime \prime \prime}(x) \geq 0, x \geq 0$, and implies finally that $W_{q}^{\prime}(x)$ is convex, with a unique minimum.
} 
The corresponding barrier function is

$$
H_{w}(b):=\frac{1-G_{w}^{\prime}(b)}{W_{q}^{\prime}(b)}, b \geq 0
$$

The most important cases of bail-out costs $w(x)$ are

1. exponential $w(x)=e^{\theta x}$, when $G_{w}(x)=Z_{q}(x, \theta)$ (Prop. 7.2), and

2. linear $w(x)=k x-K$. For $x<0$, the constants $k>0$ and $K \in \mathbb{R}$ may be viewed as proportional and fixed bail-out costs, respectively. ${ }^{31}$ In this case as well, $G_{w}(x)$ may be obtained by using $Z_{q}(x, \theta)$ as generating function in $\theta$, i.e. the coefficients of $K, k$ in $G_{w}(x)$ are found by differentiating with respect to $\theta$ the $Z_{q}(x, \theta)$ scale function 0 and 1 times respectively, and taking $\theta=0$. This yields

$$
G_{w}(x)=k Z_{q}^{(1)}(x)-K Z_{q}(x)
$$

where $Z_{q}^{(1)}(x)$ is given by (5.6). In the simple, but important particular case $w(x)=-K$, the modified de Finetti value function and barrier function are respectively

$$
\begin{aligned}
V_{K}^{b]}(x) & =-K Z_{q}(x)+W_{q}(x) \frac{1+K Z_{q}^{\prime}(b)}{W_{q}^{\prime}(b)}, \\
H_{K}(b) & :=\frac{1}{W_{q}^{\prime}(b)}+K \frac{Z_{q}^{\prime}(b)}{W_{q}^{\prime}(b)}=\frac{1+K q W_{q}(b)}{W_{q}^{\prime}(b)} .
\end{aligned}
$$

Remark 9.1. Optimality largely rests on the sign of the numerator

$$
H_{w}^{\prime}(b)=\frac{-W_{q}^{\prime \prime}(b)+\left(G_{w}^{\prime} W_{q}^{\prime \prime}-W_{q}^{\prime} G_{w}^{\prime \prime}\right)(b)}{\left(W_{q}^{\prime}\right)^{2}(b)} .
$$

For (9.7) for example,

$$
H_{K}^{\prime}(b)=\frac{K q \Delta_{q}^{(W)}(b)-W_{q}^{\prime \prime}(b)}{\left(W_{q}^{\prime}\right)^{2}(b)},
$$

where

$$
\Delta_{q}^{(W)}(b):=\left(\left(W_{q}^{\prime}\right)^{2}-W_{q} W_{q}^{\prime \prime}\right)(b)=\left(W_{q}^{\prime}\right)^{2}(b) \frac{\mathrm{d}}{\mathrm{d} b}\left(\frac{W_{q}}{W_{q}^{\prime}}\right)(b)
$$

Since the excursion rate $\nu(b)=\frac{W_{q}^{\prime}}{W_{q}}(b)$ is by definition decreasing (see Rem 3.3), it follows that $\Delta_{q}^{(W)}(b) \geq 0 .{ }^{32}$

\footnotetext{
${ }^{31}$ The cases $k \in(0,1]$ and $k>1$ correspond to management being held responsible for only part of the deficit at ruin, and to having to pay extra costs at liquidation, respectively. When $K<0$, early liquidation is rewarded; when $K>0$, late ruin is rewarded.

${ }^{32}$ Incidentally, when $\sigma>0$, this is also implied by the creeping drawdown law [118] ([98], (2.5)):
}

$$
\mathbb{E}_{x}\left[e^{-q \tau_{a}} ; Y_{\tau_{a}}=a\right]=\frac{\sigma^{2}}{2} \frac{\Delta_{q}^{(W)}(a)}{W_{q}^{\prime}(a)}, \forall x
$$


Let $b_{0}$ denote the last maximum of the unconstrained $H_{D}(b)$, and, $\forall b \geq b_{0}$, let

$$
K(b)=\frac{W_{q}^{\prime \prime}(b)}{q \Delta_{q}^{(W)}(b)} \geq 0,
$$

denote the unique $K \geq 0$ satisfying $H_{K}^{\prime}(b)=0$.

Then, assuming complete monotonicity of the Lévy measure, Proposition 4.5 and Theorem 4.4 from [75] show that for every $b \geq b_{0} K(b)$ is strictly increasing. Therefore, barrier policies are optimal and $b$ yields the optimal barrier for the cost $K(b)$ (in their paper, the parameter $K$ intervenes as a Lagrange multiplier associated to a time constraint).

\subsection{Optimal de Finetti dividends barrier until Parisian ruin}

Differentiating (8.7) and using twice (5.7), we find that the optimal de Finetti dividends barrier $b$ until Parisian ruin must satisfy

$$
\theta\left(\frac{\theta}{\lambda} Z_{q}(b, \theta)-W_{q}(b)\right)=W_{q}^{\prime}(b), \quad \theta=\Phi(q+\lambda)
$$

(note that the same equation was obtained in [120] in the context of a different, but equivalent problem involving running costs).

When $\lambda \rightarrow \infty$, the LHS of (9.12) converges to $W_{q}^{\prime \prime}(b)+W_{q}^{\prime}(b)$ by (13.4). Thus, $\lim _{\lambda \rightarrow \infty} b_{\lambda}^{*}=b^{*}$, recovering the classic optimality equation.

An important case is that when the optimal dividends barrier is 0 ; this may be viewed as a measure of the process involved corresponding to an "efficient company" (ready to pay dividends) - see [18]. The "efficiency" condition here is

$$
\Phi(q+\lambda)\left(\frac{\Phi(q+\lambda)}{\lambda}-W_{q}(0)\right) \geq W_{q}^{\prime}(0)
$$

see also [135].

\subsection{The Shreve-Lehoczky-Gaver infinite horizon objective, with linear penalties}

We turn now to an objective which was first considered in a diffusion setting by Shreve, Lehoczky, and Gaver (SLG) $[140]$ - see also $[46,113]$ - to be called SLG objective.

Suppose a subsidiary must be bailed out each time its surplus is negative, and assume the penalty costs are linear $w(x)=k x$. The optimization objective of interest combines discounted dividends $U_{t}$, and cumulative bailouts $L_{t}$

$$
\begin{aligned}
& V_{S, k}(x)=\sup _{\pi} V_{S, k}^{\pi}(x), \\
& V_{S, k}^{\pi}(x)=\mathbb{E}_{x}^{\pi}\left[\int_{0}^{\infty} e^{-q t} \mathrm{~d} U_{t}^{\pi}-k \int_{0}^{\infty} e^{-q t} \mathrm{~d} L_{t}^{\pi}\right]
\end{aligned}
$$

where $\pi$ is a dividend/bailout policy, and $k \geq 1$.

Importantly, for Lévy processes the optimal dividend/bailout policy $\pi$ is always of constant barrier type [31], and the objective for fixed $b$ has the simple expressions provided in (4.3) and (4.4) in [31] (and included 
above as (6.2), Thm. 6.1 and (6.27), Thm. 6.15), resulting in: ${ }^{33}$

$$
\begin{aligned}
V_{S, k}^{[0, b]}(x) & =V^{[0, b]}(x)-k B^{[0, b]}(x)=\frac{Z_{q}(x)}{Z_{q}^{\prime}(b)}+k\left(Z_{q}^{(1)}(x)-\frac{Z_{q}(x)}{Z_{q}^{\prime}(b)}\left(Z_{q}^{(1)}\right)^{\prime}(x)\right) \\
& =k\left(\bar{Z}_{q}(x)+\frac{\kappa^{\prime}\left(0_{+}\right)}{q}\right)+Z_{q}(x) H_{k}^{S L G}(b),
\end{aligned}
$$

with barrier function

$$
H_{k}^{S L G}(b)=\frac{1-k Z_{q}(b)}{q W_{q}(b)}
$$

- see also Proposition 3.1 from [148] for a generalization involving fixed dividend costs $K$. This impulse control problem involves replacing the reflection barrier by a $b_{1}, b_{2}$ band. It turns out that the value function is of the same form, but the barrier function changes, to

$$
H_{k, K}^{S L G}(b)=\frac{b_{1}-b_{2}-K-k\left(\bar{Z}_{q}\left(b_{2}\right)-\bar{Z}_{q}\left(b_{1}\right)\right)}{Z_{q}\left(b_{2}\right)-Z_{q}\left(b_{1}\right)} .
$$

Note that the derivation becomes simpler than in the reflection case.

The next proposition merges new results from Proposition 1 in [27] with previously known results from Lemma 2 in [31]. The main object is the function $k_{f}:[0, \infty) \rightarrow\left[k_{0}, \infty\right)$ defined by

$$
\begin{aligned}
& k_{f}(b):=\frac{W_{q}^{\prime}(b)}{Z_{q}(b) W_{q}^{\prime}(b)-q W_{q}^{2}(b)}, \quad b>0, \\
& k_{0}:=k_{f}\left(0_{+}\right)=\frac{W_{q}^{\prime}\left(0_{+}\right)}{\left.W_{q}^{\prime}\left(0_{+}\right)-q W_{q}^{2}\left(0_{+}\right)\right)}= \begin{cases}1, & \text { if } X \text { is of unbounded variation, } \\
1+\frac{q}{\Pi(0, \infty)}, & \text { if } X \text { is of bounded variation. }\end{cases}
\end{aligned}
$$

This function is increasing, by the well known identity ([22], Thm. 1) $)^{34}$

$$
\mathbb{E}_{x}\left[\mathrm{e}^{-q \tau^{b}}\right]=Z_{q}(x)-q \frac{W_{q}(b)}{W_{q}^{\prime}(b)} W_{q}(x) \Longrightarrow k_{f}(b)=\frac{1}{\mathbb{E}_{b}\left[\mathrm{e}^{-q \tau^{b}}\right]},
$$

and since the map $b \mapsto \mathbb{E}_{b}\left[\mathrm{e}^{-q \tau^{b}}\right]$ is decreasing.

The monotonicity allows us to re-parametrize the problem in terms of the optimal barrier $b_{k}$ associated to a fixed cost $k$.

Proposition 9.2. Assume $X$ is a SNLP and $K=0$. We have the following results:

1. For fixed $x, b$, the function $k \mapsto V_{k}^{0, b}(x)$ defined in (9.14) is non-increasing.

2. For $k=k_{f}(b)$, the value function defined in (9.14) can be written as follows:

$$
V_{k_{f}(b)}^{0, b}(x)=k_{f}(b)\left[\bar{Z}_{q}(x)+\frac{p}{q}-Z_{q}(x) V^{b]}(b)\right]=k_{f}(b)\left[Z_{q}^{(1)}(x)+Z_{q}(x)\left(\frac{p}{q}-\frac{W_{q}(b)}{W_{q}^{\prime}(b)}\right)\right],
$$

where $Z_{q}^{(1)}(x)$ is defined in (5.6), and $V^{b]}(b)$ is the de Finetti objective when starting at the barrier.

\footnotetext{
${ }^{33}$ As already noted in Remark 6.17, this has the same form as the de Finetti objective (9.6) with $Z$ replacing $W$.

${ }^{34}$ Some papers refer to this as the log-convexity of $Z_{q}(x)$.
} 
3. For fixed $k$, the barrier function $H_{k}^{S L G}$ defined in (9.15) is an increasing-decreasing function with a unique maximum $b_{k} \geq 0$. Moreover, if $b_{k}>0$, then $k_{f}\left(b_{k}\right)=k$.

Proof. 1. This is obvious since the Shreve, Lehoczky and Gaver value function (9.14) is decreasing in $k$, and the value function $V_{k}^{0, b}(x)$ can be seen as the maximum of $\mathbb{E}_{x}\left[\int_{0}^{\infty} \mathrm{e}^{-q t}\left(\mathrm{~d} D_{t}-k \mathrm{~d} C_{t}\right)\right]$ over control couples $(C, D)$ keeping the surplus in $[0, b]$. Since the cost functional is non-increasing in $k$, our assertion follows.

2. Recalling (9.14), we need to show that

$$
-H_{k_{f}(b)}^{S L G}(b)=k_{f}(b) V^{b}(b)
$$

Indeed, it is easy to check that the equality

$$
\frac{k Z_{q}(b)-1}{q W_{q}(b)}=k \frac{W_{q}(b)}{W_{q}^{\prime}(b)}
$$

holds for $k=k_{f}(b)$.

3. For the sake of completeness, let us reproduce this proof from Lemma 2 in [31]. The derivative of the barrier function (9.15) satisfies

$$
q \frac{H^{\prime} W_{q}^{2}}{W_{q}^{\prime}}(b)=f(b):=k \frac{\Delta_{q}^{(Z W)}(b)}{W_{q}^{\prime}(b)}-1=k \mathbb{E}_{b}\left[e^{-q \tau^{b}}\right]-1=\frac{k}{k_{f}(b)}-1,
$$

where $\Delta_{q, 0}^{(Z W)}=Z^{(q)}(b) W_{q}^{\prime}(b)-\left(Z^{(q)}\right)^{\prime}(b) W_{q}(b)$ (see (6.22)). The sign of the derivative of the barrier function (9.15) coincides therefore with that of $f(b)=k \mathbb{E}_{b}^{b]}\left[e^{-q T_{0}^{b]}}\right]-1$. Clearly the latter function $f$ is decreasing in $b$ from $\lim _{b \rightarrow 0} f(b)=\frac{k}{k_{0}}-1$ to -1 .

Remark 9.3. We may conclude therefore that if

$$
k \leq k_{0} \Leftrightarrow f(0) \leq 0 \Leftrightarrow \frac{W_{q}^{\prime}(0)}{\left.W_{q}^{\prime}(0)-q W_{q}^{2}(0)\right)} \geq k,
$$

then $b_{k}=0$ is the optimal barrier, and otherwise there is a unique global and local maximum satisfying

$$
\frac{W_{q}^{\prime}\left(b_{k}\right)}{\left.Z_{q}\left(b_{k}\right) W_{q}^{\prime}\left(b_{k}\right)-q W_{q}^{2}\left(b_{k}\right)\right)}=k=\widetilde{\delta}_{q}^{-1}\left(b_{k}\right), b_{k}>0 .
$$

Remark 9.4. The last identity in Proposition 9.2 turns out useful in establishing the so called Lokka-Zervos alternative for Brownian motion with drift - see [113], [97] - and for the Cramér-Lundberg model with exponential jumps [27]. These results state that, depending on the size of transaction costs, one of the following strategies is optimal:

1. if the cost $k$ of capital injections is below a critical point $k_{c}$, then it is optimal to pay dividends and to inject capital, according to a double-barrier strategy, meaning that ruin never occurs;

2. if the cost of capital injections is above the critical point $k_{c}$, it is optimal to use a single-barrier strategy and declare bankruptcy at the first passage below 0 .

The crucial point in these two cases is that a further identity holds which allows expressing the RHS of (9.18) in terms of the $W$ scale function, and implies

$$
V_{k_{f}\left(b^{*}\right)}^{0, b^{*}}(x)=V^{d F}(x),
$$


where $b^{*}$ denotes the optimal barrier level in de Finetti's problem.

More precisely, in the Brownian motion case, note the easily checked identities

$$
Z_{q}^{(1)}(x)+Z_{q}(x)\left(\frac{p}{q}-V^{b}(b)\right)=Z_{q}^{(1)}(x)=\frac{\sigma^{2}}{2} W_{q}(b) \Longrightarrow V_{k_{f}\left(b^{*}\right)}^{0, b^{*}}(x)=V^{d F}(x),
$$

and use then the monotonicity of $V_{k}^{S L G}(x)$ in $k$.

Similar computations establish the Lokka-Zervos alternative in the Cramér-Lundberg case with exponential claims [27].

\subsection{The dividends and penalty objective, with exponential utility}

Given $\delta, \theta, \vartheta>0$, one may consider the barrier strategy obtained by minimizing the objective (6.19). Such an objective is based on exponential utility that rewards late ruin and cumulative dividends while penalizing deficit at ruin. Recall that the barrier function of (6.19) is

$$
H_{D P}(b)=\frac{Z_{q}^{\prime}(b, \theta)+\vartheta Z_{q}(b, \theta)}{W_{q}^{\prime}(b)+\vartheta W_{q}(b)} .
$$

For $\theta=\vartheta=0$, this reduces to $q \frac{W_{q}(b)}{W_{q}^{\prime}(b)}$, which is clearly an increasing function. For $\theta=0,(6.19)$ reduces to a dividends and time objective, with barrier function

$$
H_{D T}(b)=\frac{Z_{q}^{\prime}(b)+\vartheta Z_{q}(b)}{W_{q}^{\prime}(b)+\vartheta W_{q}(b)} .
$$

This bounded function, with values in between $H_{D T}(0)=\frac{q W_{q}(0)+\vartheta}{W_{q}^{\prime}(0)+\vartheta W_{q}(0)}$, and $H_{D T}(\infty)=\frac{q+\vartheta \frac{q}{\Phi_{q}}}{\Phi_{q}+\vartheta}$, is the barrier function of the objective

$$
D T^{b}(x, \vartheta):=\mathbb{E}_{x}^{b]}\left[e^{-q T_{0}^{b]}-\vartheta U} T_{0}^{b]}\right]=\mathbb{E}_{x}^{b]}\left[e^{-\vartheta U} T_{0}^{b]} ; T_{0}^{b]}<e_{q}\right]=Z_{q}(x)-W_{q}(x) \frac{Z_{q}^{\prime}(b)+\vartheta Z_{q}(b)}{W_{q}^{\prime}(b)+\vartheta W_{q}(b)} .
$$

Remark 9.5. Note that this objective encourages taking dividends soon; in fact, everything is lost at $e_{q}$, which must be interpreted as a catastrophic event. An alternative would be to minimize $\mathbb{E}_{x}^{b]}\left[e^{-\vartheta U_{0}^{b]} \wedge e_{q}}\right]$, which would also encourage taking dividends soon, but with less urgency. The optimal barrier for this last objective should increase with respect to that of (9.23).

Remark 9.6. The sign of the derivative of the barrier function (9.22) of the exponentiated dividends and time objective (9.23) is determined by

$$
\left(Z_{q}^{\prime \prime}(x)+\vartheta Z_{q}^{\prime}(x)\right)\left(W_{q}^{\prime}(x)+\vartheta W_{q}(x)\right)-\left(W_{q}^{\prime \prime}(x)+\vartheta W_{q}^{\prime}(x)\right)\left(Z_{q}^{\prime}(x)+\vartheta Z_{q}(x)\right) \cdot{ }^{35}
$$

Some numerical results involving the exponential utility barrier functions (6.20), (9.22) and their critical points are presented in Section 10.4. We have never found multi-modal instances, suggesting that the optimal policy is simpler to implement than that for the de Finetti objective.

\footnotetext{
${ }^{35}$ Even after simplification

$$
q\left(\vartheta^{2}\left(W_{q}(x)^{2}-W_{q}^{\prime}(x) \bar{W}_{q}(x)\right)+\vartheta\left(W_{q}^{\prime}(x) W_{q}(x)-W_{q}^{\prime \prime}(x) \bar{W}_{q}(x)\right)+W_{q}^{\prime}(x)^{2}-W_{q}^{\prime \prime}(x) W_{q}(x)\right)-\vartheta\left(W_{q}^{\prime \prime}(x)+\vartheta W_{q}^{\prime}(x)\right),
$$
}

this seems hard to analyze. 
Remark 9.7. For comparison with (9.23), consider also the linearized value function (see Thms. $6.18 \mathrm{C}$ ) and $6.1 \mathrm{~A}))$

$$
\begin{aligned}
\mathbb{E}_{x}^{b]}\left[q T_{0}^{b]}+\vartheta U_{T_{0}^{b]}}\right] & =q\left(W(x) \frac{W(b)}{W^{\prime}(b)}-\int_{0}^{x} W(y) \mathrm{d} y\right)+\vartheta \frac{W(x)}{W^{\prime}(b)} \\
& =-q \int_{0}^{x} W(y) \mathrm{d} y+W(x) \frac{q W(b)+\vartheta}{W^{\prime}(b)} \frac{q W(b)+\vartheta}{W^{\prime}(b)}
\end{aligned}
$$

which needs to be maximized.

The optimization (9.23) may then be viewed as a risk sensitive optimization with exponential utility $e^{-x}$, applied to the random variable $q T_{0}^{b]}+\vartheta U_{T_{0}^{b]}}$.

\subsection{Optimization of dividends for spectrally positive processes}

The dividends of a spectrally positive process $X_{t}$ are the bailouts of its dual $-X_{t}$. Furthermore, for a fixed upper barrier $b$, the argument $x$ of the scale functions must be replaced by $b-x$. The end result for the de Finetti problem is ([39], Lem. 2.1)

$$
V(x)=Z_{q}(b-x) \frac{G_{q}^{B}(b-a)}{Z_{q}(b-a)}-G_{q}^{B}(b-x), \quad G_{q}^{B}(x)=\bar{Z}_{q}(x)+\frac{\kappa^{\prime}\left(0_{+}\right)}{q}, q>0, x \leq b .
$$

Barrier policies $b^{*}$ are always optimal, and smooth fit yields that $\bar{Z}_{q}(x)=\frac{p_{+}}{q}$ ([39], Thm. 2.1).

Since stopping happens now without overshoot, the only relevant penalty of ruin is $w(x)=-K$, and $(9.24)$ still holds, with $G_{q}^{B}(x)$ replaced by $G_{q}^{B}(x)-K([151]$, Thm. 3.1).

For Parisian observation of de Finetti dividends and a final ruin penalty $K$, the value function is given by (8.19), applied to $b-x$, and the optimal barrier must satisfy the equation ([152], (3.40), Lem. 3.6; [129], Lem. 4.2)

$$
\frac{\lambda}{q+\lambda}\left(\bar{Z}_{q}(b)-\frac{p}{q}\right)+\frac{Z_{q, \lambda}(b)}{\Phi(q+\lambda)}+K=0
$$

This has a unique positive root if and only if $\frac{\lambda}{q+\lambda} \frac{p}{q}>\frac{1}{\Phi(q+\lambda)}+K$.

For Shreve, Lehoczky and Gaver dividends with costs $k x+K$ for a capital injection of $x$, and with Parisian observation, the value function $V(x)$ ([152], Thm. 4.1) is obtained by choosing a level $a$ for capital injections and a barrier $b$, such that $V(a)=V(0)+k a+K, V^{\prime}(a)=k, V^{\prime}(b)=1$. This yields ([152], (4.10))

$$
\left\{\begin{array}{l}
Z_{q, \lambda}(b-a)=k \\
\frac{\lambda}{q+\lambda}\left(\bar{Z}_{q}(b)-\bar{Z}_{q}(b)\right)+\frac{Z_{q, \lambda}(b)-Z_{q, \lambda}(b-a)}{\Phi(q+\lambda)}=k a+K
\end{array} .\right.
$$

\section{EXAMPLES}

\subsection{Brownian motion with drift}

For Brownian motion with drift $X_{t}=\sigma B_{t}+\mu t, \mu \neq 0$ (a possible model for small claims), $\kappa(\theta)=\mu \theta+\frac{\sigma^{2}}{2} \theta^{2}$ and let $\gamma=\frac{2 \mu}{\sigma^{2}}$ be the adjustment coefficient. The roots of $\kappa(\theta)-q=0$ are $\rho_{1}=(-\mu+\mathfrak{D}) / \sigma^{2}=\Phi(q)$ and $\rho_{2}=(-\mu-\mathfrak{D}) / \sigma^{2}$ where $\mathfrak{D}=\sqrt{\mu^{2}+2 q \sigma^{2}}$. The $W$ scale function is

$$
W_{q}(x)=\frac{1}{\mathfrak{D}}\left[e^{\rho_{1} x}-e^{\rho_{2} x}\right]=\frac{1}{\mathfrak{D}}\left[e^{(-\mu+\mathfrak{D}) x / \sigma^{2}}-e^{-(\mu+\mathfrak{D}) x / \sigma^{2}}\right]=\frac{2 e^{-\mu x / \sigma^{2}}}{\mathfrak{D}} \sinh \left(x \mathfrak{D} / \sigma^{2}\right)
$$


and

$$
\bar{W}_{q}(x)=\left\{\begin{array}{ll}
\frac{1}{\mathfrak{D}}\left[\frac{e^{\lambda_{1} x}}{\lambda_{1}}-\frac{e^{\lambda_{2} x}}{\lambda_{2}}-\frac{\mathfrak{D}}{q}\right], & q>0 \\
\frac{1}{\mu}\left[x-\frac{1-e^{-\gamma x}}{\gamma}\right], & q=0
\end{array} .\right.
$$

The second scale function for $x \geq 0$ is:

$$
Z_{q}(x, \theta)=Z_{q}(x)+\theta \frac{\sigma^{2}}{2} W_{q}(x)=\frac{q-\kappa(\theta)}{\mathfrak{D}}\left[\frac{e^{\rho_{1} x}}{\rho_{1}-\theta}-\frac{e^{\rho_{2} x}}{\rho_{2}-\theta}\right] .
$$

One may check that for every $q$

$$
\Delta_{q, \theta}^{(Z W)}(x, x)=\frac{2}{\sigma^{2}} e^{-\gamma x}, \Delta_{q}^{(W)}(x)=\left(W_{q}^{\prime}\right)^{2}(x)-W_{q}(x) W_{q}^{\prime \prime}(x)=\frac{4}{\sigma^{4}} e^{-\gamma x}, \Lambda_{0}(x):=\frac{W_{0}^{\prime \prime}(x)}{\Delta_{0}^{(W)}(x)}=-\mu .
$$

Finally, the general result for reflected stopping times (4.2) yields, after some symbolic algebra manipulations, to

$$
\Psi_{q}^{b]}(x)=e^{-x \frac{\mu}{\sigma^{2}}} \frac{H(b-x)}{H(b)}, H(x)=\sqrt{2 q \sigma^{2}+\mu^{2}} \cosh \left(\frac{x \sqrt{2 q \sigma^{2}+\mu^{2}}}{\sigma^{2}}\right)-\mu \sinh \left(\frac{x \sqrt{2 q \sigma^{2}+\mu^{2}}}{\sigma^{2}}\right)
$$

see also Theorem 1.1 in [116] for a proof using martingale stopping.

Example 10.1. Theorem 6.18 becomes with $x>0$ :

(1) the expected time to ruin when $\mu<0$ is

$$
\mathbb{E}_{x}\left[T_{0}\right]=W(x) / \Phi(0)-\bar{W}(x)=\frac{1}{-\gamma \mu}\left[1-e^{-\gamma x}\right]-\frac{1}{\mu}\left[x-\frac{1-e^{-\gamma x}}{\gamma}\right]=-\frac{x}{\mu} .
$$

We can also check, as is well known, that the last result holds asymptotically for any Lévy process with $\kappa^{\prime}(0)<0$, i.e. that $\lim _{x \rightarrow \infty} \frac{\mathbb{E}_{x}\left[T_{0}\right]}{x}=-\frac{1}{\kappa^{\prime}(0)}$.

(2) When $\mu>0$, using $W^{*, 2}(x)=\mu^{-2}\left(x\left(1+e^{-\gamma x}\right)-2 \frac{1-e^{-\gamma x}}{\gamma}\right)$, we find that the expected time to ruin conditional on ruin occurring is:

$$
\begin{aligned}
& \mathbb{E}_{x}\left[T_{0} \mathbb{1}_{\left\{T_{0}<\infty\right\}}\right]=\frac{\kappa^{\prime \prime}(0)}{2 \kappa^{\prime}(0)} W(x)+\kappa^{\prime}(0) W^{* 2}(x)-\bar{W}(x) \\
& =\frac{1}{\mu \gamma}\left[1-e^{-\gamma x}\right]-\frac{1}{\mu}\left[x-\frac{1-e^{-\gamma x}}{\gamma}\right]+\mu^{-1}\left(x\left(1+e^{-\gamma x}\right)-2 \frac{1-e^{-\gamma x}}{\gamma}\right) \\
& =\frac{x}{\mu} e^{-\gamma x}
\end{aligned}
$$

with maximum at $x^{*}=\gamma^{-1}=\frac{\sigma^{2}}{2 \mu}=\frac{\kappa^{\prime \prime}(0)}{2 \kappa^{\prime}(0)}$.

This value furnishes a reasonable initial reserve, also since it coincides with the expected global infimum of a risk process started at $x^{*}$ is 0 . Indeed, assuming $\kappa^{\prime}(0)>0$ and differentiating the Wiener-Hopf factorization $\mathbb{E}_{0}\left[e^{s} \underline{X}_{\infty}\right]=\kappa^{\prime}(0) \frac{s}{\kappa(s)}$ yields

$$
\mathbb{E}_{0}\left[\underline{X}_{\infty}\right]=\kappa^{\prime}(0) \lim _{s \rightarrow 0} \frac{\kappa(s)-s \kappa^{\prime}(s)}{\kappa(s)^{2}}=\kappa^{\prime}(0) \lim _{s \rightarrow 0} \frac{-s \kappa^{\prime \prime}(s)}{2 \kappa(s) \kappa^{\prime}(s)}=\frac{-\kappa^{\prime \prime}(0)}{2 \kappa^{\prime}(0)} .
$$


Example 10.2. Optimizing the barrier under the classic de Finetti objective Theorem 6.1 A) amounts to minimizing

$$
W_{q}^{\prime}(x)=\frac{1}{\sigma^{2} \mathfrak{D}}\left[(\mathfrak{D}-\mu) e^{(\mathfrak{D}-\mu) x / \sigma^{2}}+(\mu+\mathfrak{D}) e^{-(\mu+\mathfrak{D}) x / \sigma^{2}}\right]
$$

Now the scale function verifies that

$$
\frac{\sigma^{2}}{2} W_{q}^{\prime \prime}(x)=q W_{q}(x)-\mu W_{q}^{\prime}(x)
$$

From this, it follows that if $\mu>0$, then $b^{*}$ satisfies

$$
W_{q}\left(b^{*}\right) / W_{q}^{\prime}\left(b^{*}\right)=\mu / q
$$

and is explicitly given by [73]

$$
e^{\frac{2 b^{*} \mathfrak{D}}{\sigma^{2}}}=\left(\frac{\mathfrak{D}+\mu}{\mathfrak{D}-\mu}\right)^{2} \Longrightarrow b^{*}=\frac{\sigma^{2}}{\mathfrak{D}} \log \left(\frac{\mathfrak{D}+\mu}{\mathfrak{D}-\mu}\right)=\frac{2}{\lambda_{1}-\lambda_{2}} \log \left(\frac{-\lambda_{2}}{\lambda_{1}}\right)>0 .
$$

Furthermore, as shown by Jeanblanc and Shiryaev [84], for $\mu>0$ it holds that $\frac{\sigma^{2}}{2}\left(V^{\left.b^{*}\right]}\right)^{\prime \prime}(x)+\mu\left(V^{b^{*}}\right)^{\prime}(x)-$ $q\left(V^{b^{*}}\right)(x)<0$ for $x>b^{*}$, and this implies that $\pi_{b^{*}}$ is the optimal strategy (among all admissible strategies).

If $\mu \leq 0$ on the other hand, $W_{q}^{\prime}(x)^{-1}$ attains its maximum over $[0, \infty)$ in $x=0$, and $b^{*}=0$ is optimal.

Example 10.3. Optimal de Finetti dividends barrier until Parisian ruin. Recall the equation (9.12)

$$
\frac{\Phi(q+\lambda)}{\lambda} Z_{q}(b, \Phi(q+\lambda))-W_{q}(b)=\frac{W_{q}^{\prime}(b)}{\Phi(q+\lambda)} .
$$

For Brownian motion, this yields

$$
\begin{gathered}
\Phi(q+\lambda)\left[\frac{e^{\lambda_{1} x}}{\lambda_{1}-\Phi(q+\lambda)}-\frac{e^{\lambda_{2} x}}{\lambda_{2}-\Phi(q+\lambda)}\right]-\left[e^{\lambda_{1} x}-e^{\lambda_{2} x}\right]=\frac{\left[\lambda_{1} e^{\lambda_{1} x}-\lambda_{2} e^{\lambda_{2} x}\right]}{\Phi(q+\lambda)} \\
\Longrightarrow e^{\frac{2 D b^{*}}{\sigma^{2}}}=\left(\frac{\lambda_{2}}{\lambda_{1}}\right)^{2} \frac{\Phi(q+\lambda)-\lambda_{1}}{\Phi(q+\lambda)-\lambda_{2}} \Longrightarrow b^{*}=\frac{1}{\lambda_{1}-\lambda_{2}} \log \left(\left(\frac{\lambda_{2}}{\lambda_{1}}\right)^{2} \frac{\Phi(q+\lambda)-\lambda_{1}}{\Phi(q+\lambda)-\lambda_{2}}\right)>0,
\end{gathered}
$$

which converges to (10.6) when $\lambda \rightarrow \infty$.

Example 10.4. The SLG objective Theorem 6.1 B) is studied in [31, 113]. The candidate optimal barrier (9.15) will satisfy $k \Delta_{q}^{(Z W)}(b)=W_{q}^{\prime}(b)$, which simplifies here to

$$
\cosh \left(x \mathfrak{D} / \sigma^{2}\right)-\frac{\mu}{\mathfrak{D}} \sinh \left(x \mathfrak{D} / \sigma^{2}\right)=k e^{-x \mu / \sigma^{2}} .
$$




\subsection{Scale computations for processes with rational Laplace exponent}

Generalizing the previous example, we now assume the Laplace exponent is a rational function and that the equation $\kappa(\theta)-q=0$ has distinct real roots $\lambda_{q}^{(i)}$. From the partial fraction expansion of $1 /(\kappa(\theta)-q)$, we easily obtain the $W$ scale function

$$
W_{q}(x)=\sum_{i} A_{i} e^{\lambda_{q}^{(i)} x}, q>0
$$

where $A_{i}=1 / \kappa^{\prime}\left(\lambda_{q}^{(i)}\right)$. Furthermore,

$$
\bar{W}_{q}(x)=\sum_{i} A_{i} \frac{e^{\lambda_{q}^{(i)} x}-1}{\lambda_{q}^{(i)}}=\sum_{i} A_{i} \frac{e^{\lambda_{q}^{(i)} x}}{\lambda_{q}^{(i)}}-\frac{1}{q},
$$

by using $\sum_{i} \frac{A_{i}}{\theta-\lambda_{q}^{(i)}}=\frac{1}{\kappa(\theta)-q}$ with $\theta=0$. Then, from (5.4) and (5.6)

$$
Z_{q}(x)=q \sum_{i} A_{i} \frac{e^{\lambda_{q}^{(i)} x}}{\lambda_{q}^{(i)}}, \quad Z_{q}^{1}(x)=q \sum_{i} A_{i} \frac{e^{\lambda_{q}^{(i)} x}}{\left(\lambda_{q}^{(i)}\right)^{2}}-\kappa^{\prime}(0) \sum_{i} A_{i} \frac{e^{\lambda_{q}^{(i)} x}}{\lambda_{q}^{(i)}}
$$

where $Z_{q}^{1}(0)=0$ holds since $\sum_{i} \frac{A_{i}}{\left(\theta-\lambda_{q}^{(i)}\right)^{2}}=\frac{\kappa^{\prime}(\theta)}{(\kappa(\theta)-q)^{2}}$ with $\theta=0$ implies $\sum_{i} \frac{A_{i}}{\left(\lambda_{q}^{(i)}\right)^{2}}=\frac{\kappa^{\prime}(0)}{q^{2}}$. Similarly, from (5.2) we obtain

$$
\begin{aligned}
Z_{q}(x, \theta) & =e^{\theta x}+(q-\kappa(\theta)) \sum_{i} A_{i} \frac{e^{\lambda_{q}^{(i)} x}-e^{\theta x}}{\lambda_{q}^{(i)}-\theta}=(\kappa(\theta)-q) \sum_{i} \frac{A_{i}}{\theta-\lambda_{q}^{(i)}} e^{\lambda_{q}^{(i)} x} \\
& =Z_{q}(x)+\theta \sum_{i} A_{i} \frac{\frac{\kappa(\theta)}{\theta}-\frac{q}{\lambda_{q}^{(i)}}}{\theta-\lambda_{q}^{(i)}} e^{\lambda_{q}^{(i)} x} .
\end{aligned}
$$

For $q=0$ the formulas are slightly different due to the fact that zero is one solution of $\kappa(\theta)=0$.

\subsection{Cramér-Lundberg model with exponential jumps}

We analyze now the Cramér-Lundberg model with exponential jump sizes with mean $1 / \mu$, jump rate $\lambda$, premium rate $c>0$, and Laplace exponent $\kappa(\theta)=\theta\left(c-\frac{\lambda}{\mu+\theta}\right)$, assuming $\kappa^{\prime}(0)=c-\frac{\lambda}{\mu} \neq 0$. Let $\gamma=\mu-\lambda / c$ denote the adjustment coefficient, and let $\lambda=\frac{\lambda}{c \mu}$. Solving $\kappa(\theta)-q=0$ for $\theta$ yields two distinct solutions $\lambda_{2} \leq 0 \leq \lambda_{1}=\Phi(q)$ given by

$$
\begin{aligned}
& \lambda_{1}=\frac{1}{2 c}\left(-(\mu c-\lambda-q)+\sqrt{(\mu c-\lambda-q)^{2}+4 \mu q c}\right), \\
& \lambda_{2}=\frac{1}{2 c}\left(-(\mu c-\lambda-q)-\sqrt{(\mu c-\lambda-q)^{2}+4 \mu q c}\right) .
\end{aligned}
$$

The $W$ scale function and its integral are:

$$
W_{q}(x)=A_{1} e^{\lambda_{1} x}+A_{2} e^{\lambda_{2} x}, \bar{W}_{q}(x)=\left\{\begin{array}{ll}
\frac{1}{\kappa^{\prime}(0)}\left[x-\lambda \frac{1-e^{-\gamma x}}{\gamma}\right], & q=0 \\
A_{1} \frac{e^{\lambda_{1} x}-1}{\lambda_{1}}+A_{2} \frac{e^{\lambda_{2} x}-1}{\lambda_{2}}, & q>0
\end{array},\right.
$$


where $A_{1}=c^{-1}\left(\mu+\lambda_{1}\right)\left(\lambda_{1}-\lambda_{2}\right)^{-1}=1 / \kappa^{\prime}\left(\lambda_{1}\right)$ and $A_{2}=-c^{-1}\left(\mu+\lambda_{2}\right)\left(\lambda_{1}-\lambda_{2}\right)^{-1}=1 / \kappa^{\prime}\left(\lambda_{2}\right)$. Using the general results of the previous example, we find

$$
Z_{q}(x)=\left\{\begin{array}{ll}
1 & q=0 \\
q\left(\frac{A_{1}}{\lambda_{1}} e^{\lambda_{1} x}+\frac{A_{2}}{\lambda_{2}} e^{\lambda_{2} x}\right)=-\frac{c}{\mu}\left(\lambda_{2} A_{1} e^{\lambda_{1} x}+\lambda_{1} A_{2} e^{\lambda_{2} x}\right) . & q>0
\end{array} .\right.
$$

By tedious simplification of (10.7), we find that

$$
Z_{q}(x, \theta)=Z_{q}(x)+\frac{\lambda}{c} \frac{\theta}{\theta+\mu} \frac{e^{\lambda_{1} x}-e^{\lambda_{2} x}}{\lambda_{1}-\lambda_{2}}, Z_{q}^{1}(x)= \begin{cases}\lambda \frac{1-e^{-\gamma x}}{\gamma^{\gamma}}, & q=0 \\ \frac{\lambda}{\mu c} \frac{e^{\lambda_{1}}-e^{\lambda_{2} x}}{\lambda_{1}-\lambda_{2}} & q>0\end{cases}
$$

Example 10.5. Theorem 6.18 becomes:

(1) When $\kappa^{\prime}(0)<0$, we have $\Phi(0)=\zeta_{0}^{(1)}=-\gamma$ and hence

$$
\mathbb{E}_{x}\left[T_{0}\right]=-\frac{1}{\gamma} W(x)-\bar{W}(x)=-\frac{1}{\gamma c(1-\rho)}\left(1-\rho e^{-\gamma x}\right)-\frac{1}{\kappa^{\prime}(0)}\left(x-\lambda \frac{1-e^{-\gamma x}}{\gamma}\right)=-\frac{x}{\kappa^{\prime}(0)}-\frac{1}{\gamma} .
$$

(2) When $\kappa^{\prime}(0)>0$, using $W^{*, 2}(x)=\frac{\gamma x-2 \rho}{\kappa^{\prime}(0)^{2} \gamma}+\frac{e^{-x \gamma} \rho(\gamma \rho x+2)}{\kappa^{\prime}(0)^{2} \gamma}$, we find that the expected time to ruin conditional on ruin occurring is:

$$
\mathbb{E}_{x}\left[T_{0} \mathbb{1}_{\left\{T_{0}<\infty\right\}}\right]=\frac{\kappa^{\prime \prime}(0)}{2 \kappa^{\prime}(0)} W(x)+\kappa^{\prime}(0) W^{* 2}(x)-\bar{W}(x)=\frac{\rho}{c^{2} \gamma} e^{-\gamma x}(\lambda x+c),
$$

with maximum at $x^{*}=\frac{1}{\gamma}\left(2-\lambda^{-1}\right)$. This value furnishes a possible lower bound for the initial reserve, which is positive if and only if $c<2 \frac{\lambda}{\mu} \Leftrightarrow p<\frac{\lambda}{\mu}$.

Example 10.6. Let us recall now that the function $W_{q}^{\prime}(x)=H_{D}(x)^{-1}$ is unimodal with global minimum at

$$
b^{*}=\frac{1}{\lambda_{1}-\lambda_{2}} \begin{cases}\log \frac{\left(\lambda_{2}\right)^{2}\left(\mu+\lambda_{2}\right)}{\left(\lambda_{1}\right)^{2}\left(\mu+\lambda_{1}\right)} & \text { if } W_{q}^{\prime \prime}(0)<0 \Leftrightarrow(q+\lambda)^{2}-c \lambda \mu<0 \\ 0 & \text { if } W_{q}^{\prime \prime}(0) \geq 0 \Leftrightarrow(q+\lambda)^{2}-c \lambda \mu \geq 0\end{cases}
$$

since $\left.W_{q}^{\prime \prime}(0) \sim\left(\lambda_{1}\right)^{2}\left(\mu+\lambda_{1}\right)-\left(\lambda_{2}\right)^{2}\left(\mu+\lambda_{2}\right) /\left(\lambda_{1}\right)-\lambda_{2}\right)=(q+\lambda)^{2}-c \lambda \mu$ (see also (3.10)). Furthermore, the optimal strategy is always the barrier strategy at level $b^{*}[31]$.

\subsection{Numerical optimization of dividends for the Azcue-Muller example}

Consider the Cramér-Lundberg model perturbed by Gaussian component, $X_{t}=x+c t-\sum_{i=1}^{N_{t}^{(\lambda)}} C_{i}+\sigma B_{t}$, where $C_{i}$ are iid pure Erlang claims, $E_{2,1}$ of order $n=2$ and $N^{(\lambda)}$ is an independent Poisson process with arrival rate $\lambda$. The Laplace exponent is $\kappa(\theta)=c \theta-\lambda+\lambda\left(\frac{\mu}{\mu+\theta}\right)^{2}+\frac{\sigma^{2}}{2} \theta$, and the equation $\kappa(\theta)-\delta=0$ has four roots. In what follows, the choice of parameters will be such that these roots are distinct. Since $\kappa$ is a rational function, the results of Section 10.2 can be used to obtain scale functions.

The interest in this example was awakened by Azcue and Muller [35], who showed that the barrier dividend strategy is not optimal for certain parameter values. It was shown later that this is the case when the barrier function has two local maxima, and the last one is not the global maximum - see Figure 1 in [101].

It is natural to ask whether the barrier function (6.20) can have the property of multi-modality which complicates the management of dividends. We did not find any such example in our experiments presented below. 

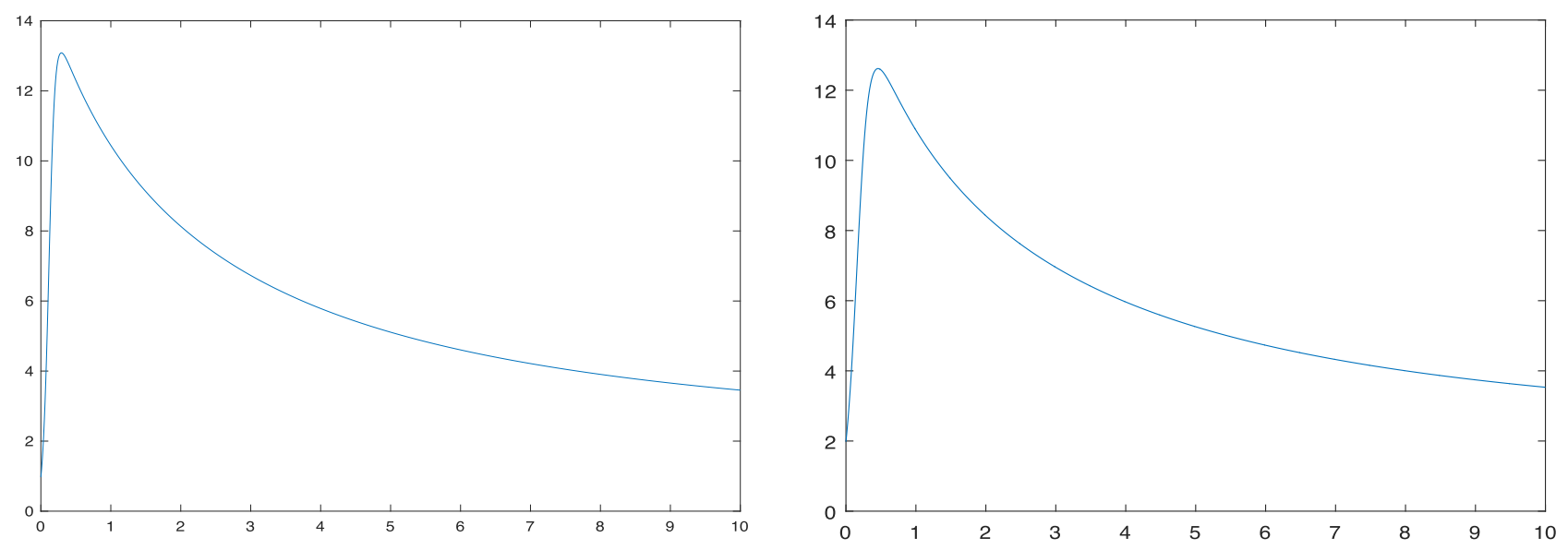

Figure 3. Left: $\sigma=1.4, \theta=-0.01, \vartheta=1$ with $H_{D P}(0)=0.98, H_{D P}(\infty)=2.5544$, Right: $\sigma=2, \theta=-0.01, \vartheta=1$ with $H_{D P}(0)=2, H_{D P}(\infty)=2.5821$.
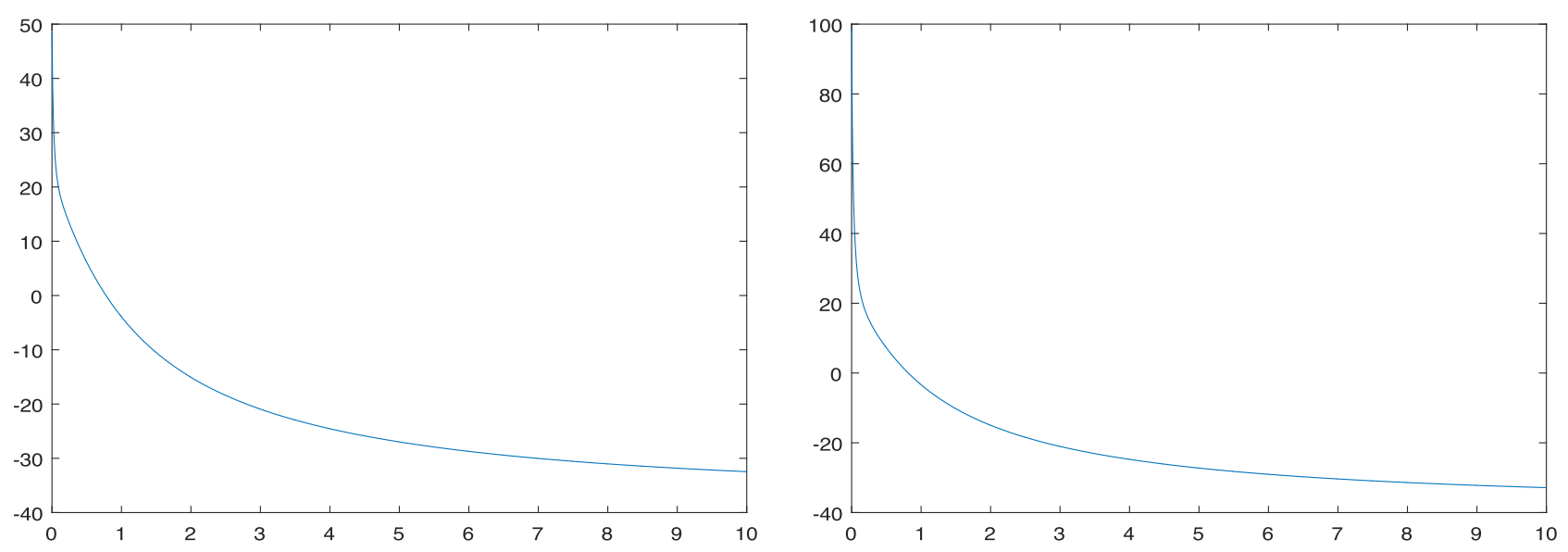

Figure 4. Left: $\sigma=1.4, \theta=-0.5, \vartheta=50$ with $H_{D P}(0)=49, H_{D P}(\infty)=2.5544$, Right: $\sigma=2$, $\theta=-0.5, \vartheta=50$ with $H_{D P}(0)=100, H_{D P}(\infty)=2.5821$.

We present now some numerical experiments using a choice of parameters close to [101], namely $\mu=1$, $\lambda=10, c=\frac{107}{5}$ and $q=\frac{1}{10}$. We consider $\sigma=1.4$ and $\sigma=2$ as given in [101]. Note that, with these choice of parameters and in the absence of Brownian component, this example corresponds to the example given by Azcue and Muler [35] for which sufficient conditions for optimal barrier strategy do not hold.

Concerning the performance of barrier strategies under the model given above, see Figures 3 and 4 , where we provide typical plots of the barrier function (6.20) of (6.19), for different values of $\vartheta>0, q>0, \theta<0$. Recall that, for $\theta=0,(6.20)$ reduces to (9.22) which is the barrier function of (9.23). Furthermore, plots of (9.22) are presented in Figures 5 and 6. 

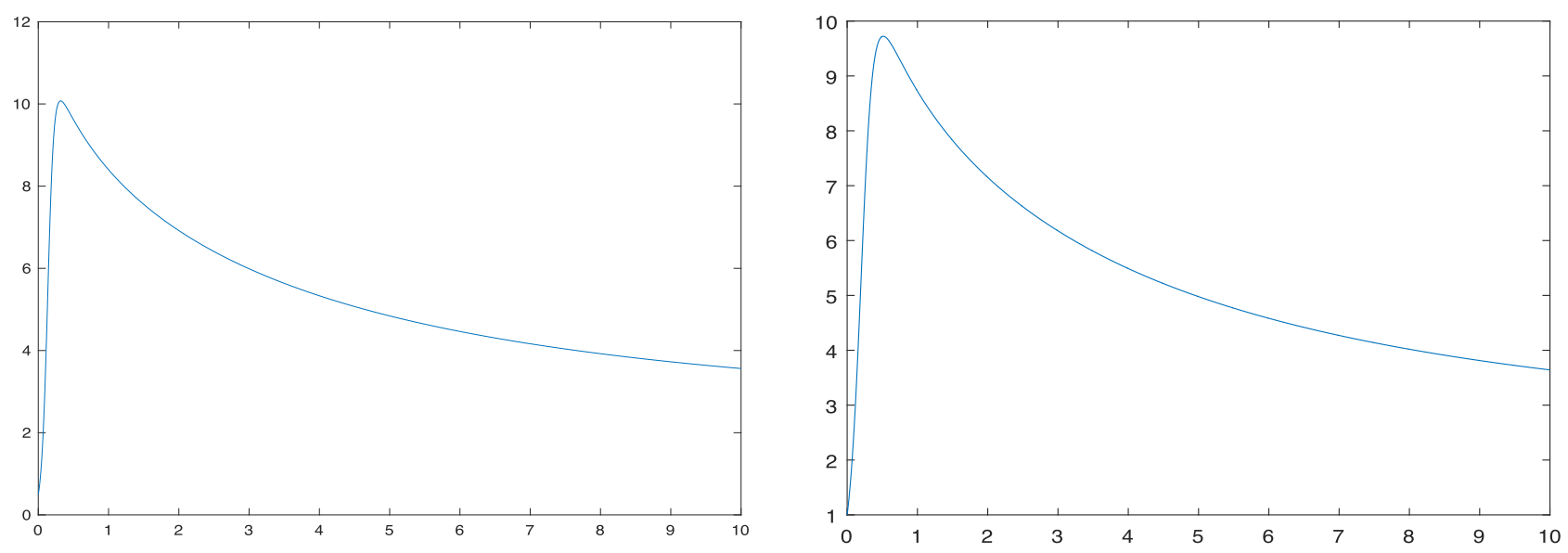

Figure 5. Left: $\sigma=1.4, \vartheta=0.5$ with $H_{D T}(0)=0.49, H_{D T}(\infty)=2.5544$, Right: $\sigma=2, \vartheta=0.5$ with $H_{D T}(0)=1, H_{D T}(\infty)=2.5821$.
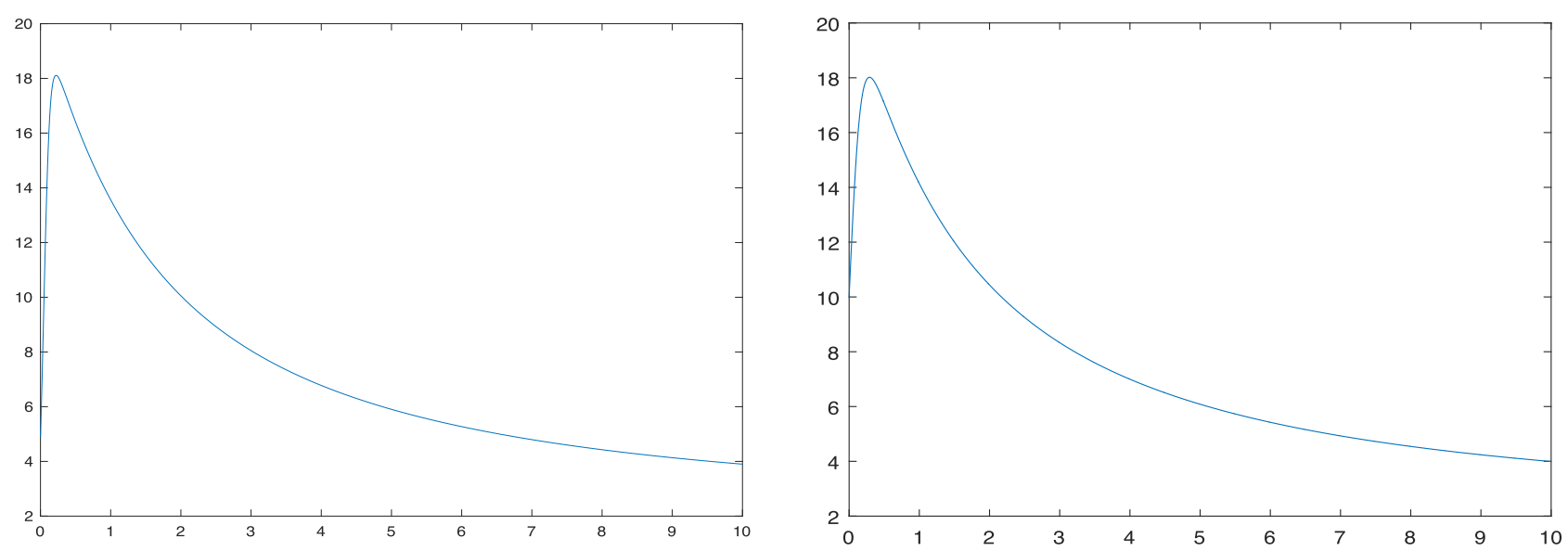

Figure 6. Left: $\sigma=1.4, \vartheta=5$ with $H_{D T}(0)=4.9, H_{D T}(\infty)=2.5544$, Right: $\sigma=2, \vartheta=5$ with $H_{D T}(0)=10, H_{D T}(\infty)=2.5821$.

\section{Strong Markov processes With Generalized DraWdown STOPPING}

In this section, $X_{t}$ will denote a one dimensional strong Markov process without positive jumps, defined on a filtered probability space $\left(\Omega,\left\{\mathcal{F}_{t}\right\}_{t \geq 0}, P\right)$.

Since many results for spectrally negative Lévy and diffusion processes require not much more than the strong Markov property, it was natural to attempt to extend such results to spectrally negative strong Markov processes. As expected, everything worked out almost smoothly for "Lévy -type cases" like random walks [19], Markov additive processes [78], Lévy processes with $\Omega$ state dependent killing [78], and there are also some results for the more challenging case of Lévy processes with state dependent drift [55]. In fact, the existence of some functions $W, Z$ satisfying (1.3), (1.11) is clear in general, by smooth crossing and the strong Markov property. However, prior to the pioneering [100], the classic and drawdown first passage literatures were restricted mostly to parallel treatments of the two particular cases of diffusions and of spectrally negative Lévy processes. [100] 
showed that a direct unified approach (inspired by [95] in the case of diffusions) may achieve the same results for all time homogeneous Markov processes.

The crux of the approach is to replace $W, Z$ in the state dependent case by differential versions $\nu$ and $\delta$, which were denoted in [100] by $b, c$, in the context of the study of drawdowns. Later, in [30], they were extended to generalized drawdown times (which include first passage times). As will be clear from the discussion below, $\nu$ and $\delta$ capture the behavior of excursions of the process away from its running maximum. Note however that $\nu$ is a measure, and determining when it admits a density requires quite different technical treatments for spectrally negative Lévy and diffusion processes (see for example Lem. 8.2 in[94] which relates this to the challenging issue of the differentiability of $W_{q}$ ); note also that computing $W, Z, \nu, \delta$ is still an open problem, even for simple classic processes like the Ornstein-Uhlenbeck process and the Feller branching diffusion with jumps. [100] (and [30]) cut through this Gordian node by restricting to processes for which the limits defining $\nu, \delta$ exist - see Assumptions (11.14), (11.15), and leaving to the user's responsibility to check this for their process; they also showed that the known results for diffusions and spectrally negative Lévy processes were just particular cases of their general formulas - see Section 11.3.

The results of $[30,100]$ provide a unifying umbrella for Lévy processes, diffusions, branching processes (including with immigration), logistic branching processes, etc., under the caveat that beyond the Lévy and diffusion cases, the user must establish the validity of Assumptions (11.14), (11.15) and manage computing $\nu, \delta$.

The end result is that for non-homogeneous spectrally negative Markov processes with classic first passage stopping we may provide extensions of the two-sided exit equalities (1.3), (1.4) and similar, involving now scale functions with one more variable

$$
\bar{\Psi}_{q}^{b}(x, a)=\frac{W_{q}(x, a)}{W_{q}(b, a)}, \quad \Psi_{q}^{b}(x, a)=Z_{q}(x, a, \theta)-W_{q}(x, a) \frac{Z_{q}(b, a, \theta)}{W_{q}(b, a)} .
$$

For diffusions for example, $W_{q}(x, a)$ is a certain Wronskian (see [47]) and for Langevin type processes with decreasing state-dependent drifts, $W_{q}(x, a)$ solves a certain renewal equation [55]. So, formally the spectrally negative Markov case is similar to the Lévy one, up to adding one variable to the fundamental functions.

Extensions to drawdown stopping are possible as well [30, 100], but they are easier to state in terms of differential exit parameters $\nu, \delta$ defined in (11.14), (11.15) below. Before reviewing these extensions, we will introduce some objects of interest via an illustrative example of first passage problem for $(X, Y)$, with $Y$ a drawdown process. In this case, simple geometric arguments (see Fig. 7) reduce the computation of Laplace transforms of exit times of $(X, Y)$ from rectangles to those of simpler Laplace transforms defined in (11.7), (11.9), which seem to be fundamental to this setup.

\subsection{Joint evolution of a strong Markov process and its drawdown in a rectangle}

In order to study the process $(X, Y)$, it is convenient to start with its evolution in a rectangular region $R:=[a, b] \times[0, d] \subset \mathbb{R} \times \mathbb{R}_{+}$, where $a<b$ and $d>0$.

A sample path of $(X, Y)$, where $X$ is chosen to be the standard Brownian motion, and the region $R$ is depicted in Figure 7.

Remark 11.1. As suggested by Figure 7, the study of the process $(X, Y)$ may be reduced to one-dimensional problems:

1. On the $y=0$ axis, we observe the maximum process $\bar{X}$. If furthermore downward excursions are excised, we obtain the so-called upward ladder process $\widetilde{X}(m)=m$ (the maximum studied as a function of itself), which is of course Markovian with generator $\frac{\partial}{\partial m}$. If furthermore time killing is present, $\widetilde{X}(m)$ becomes a killed drift subordinator, with Laplace exponent $\kappa(s)=s+\Phi_{q}$ (as a consequence of the Wiener-Hopf decomposition [94]). 


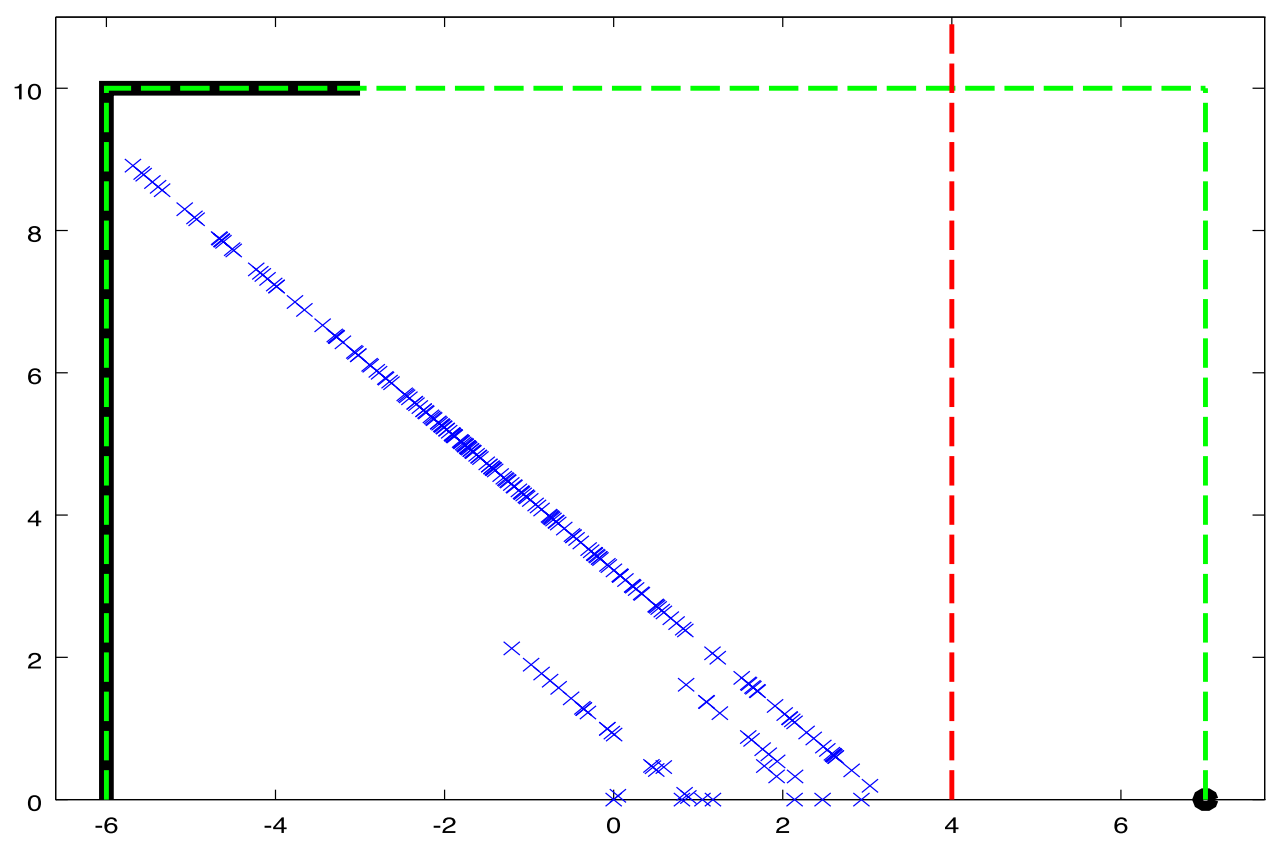

Figure 7. A sample path of $(X, Y)$ (sampled at time step $\Delta t=0.1$ ) when $X$ is a standard Brownian motion with $X_{0}=0.2$, and the region $R$ with $d=10, a=-6$ and $b=7$. The dark boundary shows the possible exit points of $(X, Y)$ from $R$. The base of the red line separates $R$ in two parts with different behavior.

2. Away from the boundary $y=0$, the process oscillates during negative excursions from the maximum on line segments $l_{\bar{X}_{t}}$ where, for $c \in \mathbb{R}, l_{c}:=\left\{x \in \mathbb{R} \times \mathbb{R}_{+}: x_{1}+x_{2}=c\right\}$. Since $\bar{X}_{t}$ is fixed during such an excursion, we are dealing here essentially with the process $-X_{t}$.

3. If the first excursion outside the rectangle kills the process, the ladder process becomes a killed drift with generator $\mathcal{G} \phi(m):=\phi^{\prime}(m)-\nu_{q}(d) \phi(m)[11,34]$, since the killing excursions are a Poisson process with rate $\nu_{q}(d)$.

4. With generalized drawdown defined in the next subsection (when the upper boundary is replaced by one determined by certain parametrizations $(\widehat{d}(m), d(m)), \widehat{d}(m)=m-d(m))$, the generator of $\widetilde{X}_{m}$ will have state dependent killing:

$$
\mathcal{G} \phi(m):=\phi^{\prime}(m)-\nu_{q}(d(m)) \phi(m)
$$

5. Finally, in the spectrally negative Markov case, the generator becomes:

$$
\mathcal{G} \phi(m):=\phi^{\prime}(m)-\nu_{q}(m, \widehat{d}(m)) \phi(m)
$$

where the killing rate

$$
\left.\nu_{q}(m, y)\right|_{y=\widehat{d}(m)}
$$

depending of both the current position and the killing limit $y=\widehat{d}(m)$ is defined in (11.14) below. 
The fact that many functionals (ruin, dividends, tax, etc.) of the original process may be expressed as functionals of the killed ladder process explains the prevalence of first order ODE's related to the generator (11.2) when working with spectrally negative processes.

We see from the remarks above that $\nu_{q}$ may serve as a more convenient alternative characteristic of a spectrally negative Markov process, replacing $W_{q}$, and that it may be used also in the case of generalized drawdown killing.

Define now

$$
T_{R}=T_{a, b, d}:=\inf \left\{t:\left(X_{t}, Y_{t}\right) \notin R\right\}=\tau_{d} \wedge T_{a,-} \wedge T_{b,+}
$$

Several implications for $T_{R}$ are immediately clear from these dynamics: for example, the process $(X, Y)$ can leave $R$ only through $\partial R \cap\left\{x \in \mathbb{R} \times \mathbb{R}_{+}: x_{1} \leq b-d\right\}$ or through the point $(b, 0)$ (see the shaded region in Fig. 7). Also,

1. If $b-a \leq d$, it is impossible for the process to leave $R$ through the upper drawdown boundary of $\partial R$ and for these parameter values $T_{R}$ reduces to $T_{a,-} \wedge T_{b,+}$. Here it suffices to know the survival/ruin functions (1.3), (1.4) in order to obtain the Laplace transform of $T_{R}$.

2. If $a+d \leq x$, it is impossible for the process to leave $R$ through the left boundary of $\partial R$, and $T_{R}$ reduces to $T_{b,+} \wedge \tau_{d}$. Here it suffices to apply the spectrally negative drawdown formulas provided in [99, 118].

3 . In the remaining case $x \leq a+d \leq b$, both drawdown and classic exits are possible. For the latter case, see Figure 7. The key observation here is that drawdown [classic] exit occurs if and only if $X_{t}$ does [does not] cross the line $x_{1}=d+a$. The final answers will combine these two cases.

Two natural objects of interest in "mixed drawdown /first passage" control over the rectangle are the "two-sided exit" times

$$
\tau_{b, d}=\min \left(\tau_{d}, T_{b,+}\right), \tau_{a, d}=\min \left(\tau_{d}, T_{a,-}\right)
$$

In terms of the two dimensional process $t \mapsto\left(X_{t}, Y_{t}\right)$, these are the first exit times from the regions $(-\infty, b) \times[0, d]$ and $(a, \infty) \times[0, d]$.

We introduce now two Laplace transforms $U b D / D b U$ (standing for up-crossing before drawdown/drawdown before up-crossing) involving the "two-sided exit" times, which are analogues of the killed survival and ruin probabilities:

$$
\begin{aligned}
U b D_{q, d}^{b}(x) & =\mathbb{E}_{x}\left[e^{-q T_{b,+}} ; T_{b,+}<\tau_{d}\right]=\mathbb{E}_{x}\left[e^{-q T_{b,+}} ; \bar{X}_{\tau_{d}}>b\right] \\
D b U_{q, \theta, d}^{b}(x) & =\mathbb{E}_{x}\left[e^{-q \tau_{d}-\theta\left(Y_{\tau_{d}}-d\right)} ; \tau_{d}<T_{b,+}\right]=\mathbb{E}_{x}\left[e^{-q \tau_{d}-\theta\left(Y_{\tau_{d}}-d\right)} ; \bar{X}_{\tau_{d}}<b\right]
\end{aligned}
$$

By using $U b D / D b U$ we provide now Laplace transforms of $T_{R}$ and of the eventual overshoot at $T_{R}$. One can break down the analysis of $T_{R}$ to nine cases, depending on which of the three exit boundaries $T_{a,-}, T_{b,+}$ or $\tau_{d}$ occurred, and on the three relations between $x, a, b$ and $d$ described above. The results are then the immediate applications of the strong Markov property.

Proposition 11.2. Consider a spectrally negative Markov process $X$ with differentiable scale function $W_{q}$. Then, for $d \geq 0$ and $a \leq x \leq b$, we have: 


\begin{tabular}{r|c|c|c} 
& $a+d \leq x \leq b$ & $x \leq a+d \leq b$ & $b \leq a+d$ \\
\hline $\mathbb{E}_{x}\left[e^{-q T_{b,+}} ; T_{b,+} \leq \min \left(\tau_{d}, T_{a,-}\right)\right]=$ & $U b D_{q, d}^{b}(x)$ & $\bar{\Psi}_{q}^{(a+d)}(x, a) U b D_{q, d}^{b}(a+d)$ & $\bar{\Psi}_{q}^{b}(x, a)$ \\
\hline $\mathbb{E}_{x}\left[e^{-q T_{a,-}+\theta\left(X_{\left.T_{a,-}-a\right)}{ }^{-a}\right.} ; T_{a,-} \leq \min \left(\tau_{d}, T_{b,+}\right)\right]=$ & 0 & $\Psi_{q, \theta}^{(a+d)}(x, a)$ & $\Psi_{q, \theta}^{b}(x, a)$ \\
\hline $\mathbb{E}_{x}\left[e^{-q \tau_{d}-\theta\left(Y_{\tau_{d}}-d\right)} ; \tau_{d} \leq \min \left(T_{b,+}, T_{a,-}\right)\right]=$ & $D b U_{q, \theta, d}^{b}(x)$ & $\bar{\Psi}_{q}^{(a+d)}(x, a) D b U_{q, \theta, d}^{b}(a+d)$ & 0
\end{tabular}

Proof. Note that in the third column the $d$ boundary is invisible and does not appear in the results, and in the first column the $a$ boundary is invisible and does not appear in the results. These two cases follow therefore by applying already known results.

The middle column holds by breaking the path at the first crossing of $a+d$. The main points here are that

1. the middle case may happen only if $X_{t}$ visits $a$ before $a+d$;

2. the first case (exit through $b$ ) and the third case (drawdown exit) may happen only if $X_{t}$ visits first $a+d$, with the drawdown barrier being invisible, and that subsequently the lower first passage barrier $a$ becomes invisible.

The results follow then due to the smooth crossing upward and the strong Markov property.

We will leave open the question of how to compute the drawdown functions $U b D / D b U$ until Section 11.3 where we will consider more general drawdown boundaries. However, we note here that for spectrally negative Lévy processes they have simple formulas. In the Lévy case for example

$$
U b D_{q, d}^{b}(x)=\mathbb{E}_{x}\left[e^{-q T_{b,+}} ; T_{b,+} \leq \tau_{d}\right]=e^{-(b-x) \frac{W_{q}^{\prime}(d)}{W_{q}(d)}}
$$

and the function $D b U$ may be obtained by integrating the fundamental law ([118], Thm. 1; [99], Thm. 3.1) ${ }^{36}$

$$
\begin{aligned}
& \delta_{q, \theta}(d, x, s):=\mathbb{E}_{x}\left[e^{-q \tau_{d}-\theta\left(Y_{\tau_{d}}-d\right)} ; \bar{X}_{\tau_{d}} \in \mathrm{d} s\right]=\left(\nu_{q}(d) e^{-\nu_{q}(d)(s-x)_{+}} \mathrm{d} s\right) \widetilde{\delta}_{q, \theta}(d) \\
& \Leftrightarrow \mathbb{E}_{x}\left[e^{-q \tau_{d}-\theta\left(Y_{\tau_{d}}-d\right)-\vartheta\left(\bar{X}_{\tau_{d}}-x\right)}\right]=\frac{\nu_{q}(d)}{\vartheta+\nu_{q}(d)} \widetilde{\delta}_{q, \theta}(d), \quad \forall x
\end{aligned}
$$

where $\widetilde{\delta}_{q, \theta}(d)$ is given by $(6.17)$. Integrating (6.16) yields

$$
D b U_{q, \theta, d}^{b}(x)=\left(1-e^{-(b-x) \frac{W_{q}^{\prime}(d)}{W_{q}(d)}}\right) \widetilde{\delta}_{q, \theta}(d) .
$$

Note that the fundamental law reflects the independence of the path before the last maximum and after, conditional on the value of the last maximum. The exponential law of the last maximum is due to the Lévy setup, and will be lost in the Markov case, where it will be replaced by the law of the first arrival in a "nonhomogeneous Poisson process of killing excursions".

Corollary 11.3. In the spectrally negative Lévy case, Theorem 11.2 holds with the first passage and drawdown functions given by (1.3), (1.4), (11.7), (11.9).

\footnotetext{
${ }^{36}$ Note that Theorem 1 in [118] give a more complicated "sextuple law" with two cases, and that Theorem 3.1 in [99] use an alternative to the function $Z_{q}(x, \theta)$, so that some computing is required to get (11.7), (6.16) and (6.21).
} 


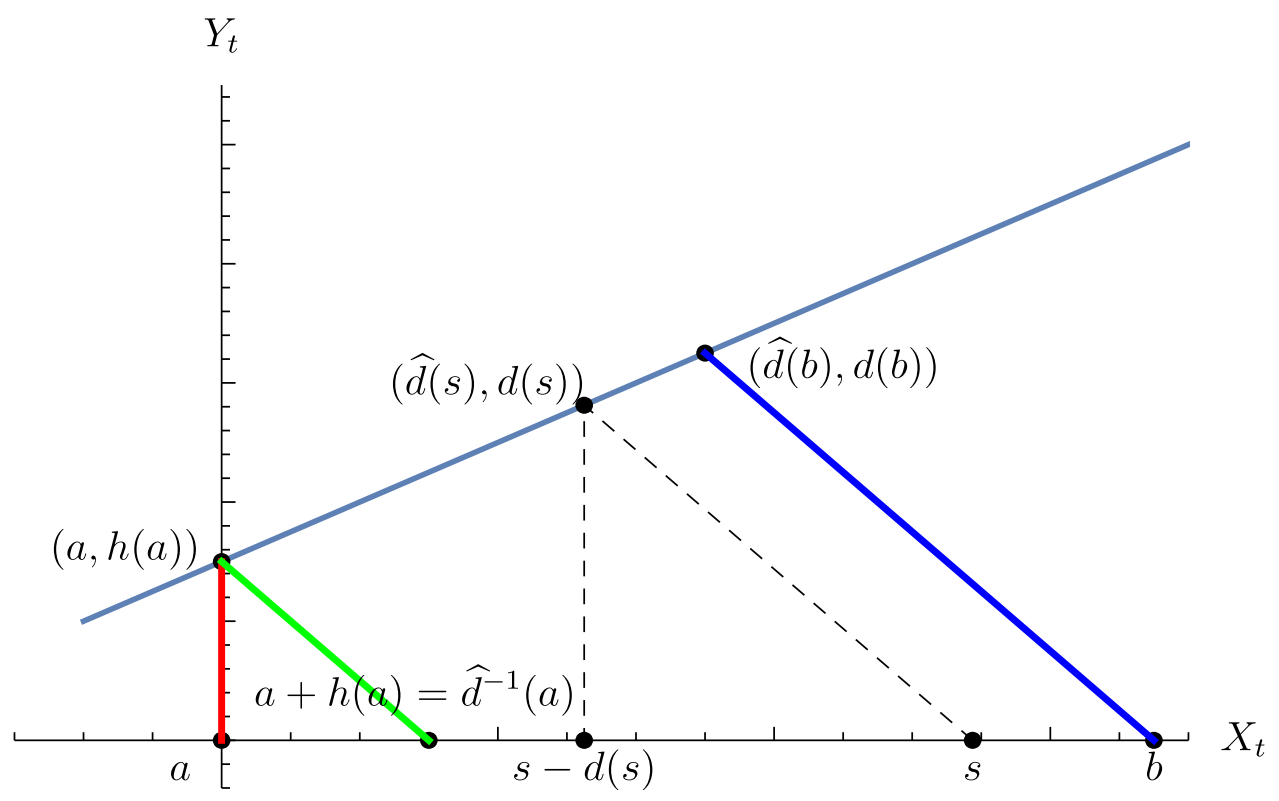

Figure 8. Affine drawdown exit of $(X, Y)$ with $a=0, d(s)=\frac{1}{3} s+1$.

\subsection{Generalized drawdown stopping for processes without positive jumps}

Generalized drawdown times appear naturally in the Azema-Yor solution of the Skorokhod embedding problem [37], and in the Dubbins-Shepp-Shiryaev, and Peskir-Hobson-Egami optimal stopping problems $[65,67,76,122]$. Importantly, they allow a unified treatment of classic first passage and drawdown times see $[34,110]$ (see also [30] for a further generalization to taxed processes). The idea is to replace the upper side of the rectangle $R$ by a parametrized curve

$$
\left(x_{1}, x_{2}\right)=(\widehat{d}(s), d(s)), \quad \widehat{d}(s)=s-d(s),
$$

where $s=x_{1}+x_{2}$ represents the value of $\bar{X}_{t}$ during the excursion which intersects the upper boundary at $\left(x_{1}, x_{2}\right)$ (see Fig. 8). Alternatively, parametrizing by $x$ yields (note $Y_{t} \geq d\left(\bar{X}_{t}\right) \Leftrightarrow Y_{t} \geq h\left(X_{t}\right)$ )

$$
y=h(x), \quad h(x)=\widehat{d}^{-1}(x)-x .
$$

Definition 11.4. [37, 110] For any function $d(s)>0$ such that $\widehat{d}(s)=s-d(s)$ is nondecreasing, a generalized drawdown time is defined by

$$
\tau_{\widehat{d}(\cdot)}:=\inf \left\{t \geq 0: Y_{t}>d\left(\bar{X}_{t}\right)\right\}=\inf \left\{t \geq 0: X_{t}<\widehat{d}\left(\bar{X}_{t}\right)\right\}
$$

Such times provide a natural unification of classic and drawdown times. Introduce

$$
\tilde{Y}_{t}:=Y_{t}-d\left(\bar{X}_{t}\right), t \geq 0
$$

to be called drawdown type process. Note that we have $\widetilde{Y}_{0}=-\widehat{d}\left(X_{0}\right)<0$, and that the process $\widetilde{Y}_{t}$ is in general non-Markovian. However, it is Markovian during each negative excursion of $X_{t}$, along one of the oblique lines in the geometric decomposition sketched in Figure 7. 
Example 11.5. With affine functions

$$
d(s)=(1-\xi) s+d \Leftrightarrow \widehat{d}(s)=\xi s-d \Leftrightarrow h(x)=\frac{(1-\xi) x+d}{\xi}, \quad \xi \in[0,1]
$$

we obtain the affine drawdown/regret times studied in [34].

Affine drawdown times reduce to a classic drawdown time (1.10) when $\xi=1, d(s)=d$, and to a time of first passage below a level when $\xi=0, \widehat{d}(s)=-d, d(s)=s+d$. When $\xi$ varies, we are dealing with the pencil of lines passing through $(x, y)=(-d, d)$. In particular, for $\xi=1$ we obtain an infinite strip, and for $\xi=0, d=0$, we obtain the positive quadrant (this case corresponds to the classic ruin time).

One of the merits of affine drawdown times is that they allow unifying the classic first passage theory with the drawdown theory [34]. A second merit is that they are optimal for the variational problem considered below.

Introduce now generalized drawdown analogues of the drawdown survival and ruin probabilities (4.9), for which we will use the same notation:

$$
\begin{aligned}
U b D_{q, \widehat{d}(\cdot)}^{b}(x) & =\mathbb{E}_{x}\left[e^{-q T_{b,+}} ; T_{b,+} \leq \tau_{\widehat{d}(\cdot)}\right] \\
D b U_{q, \theta, \widehat{d}(\cdot)}^{b}(x) & =\mathbb{E}_{x}\left[e^{-q \tau_{\widehat{d}(\cdot)}-\theta \widetilde{Y}_{\tau}(\cdot)} ; \tau_{\widehat{d}(\cdot)}<T_{b,+}\right]
\end{aligned}
$$

An extension of Theorem 11.2 to generalized drawdowns is straightforward:

Proposition 11.6. Consider a spectrally negative Markov process $X$ with differentiable scale function $W_{q}$. Then, for $a \leq x \leq b$ and $d(\cdot)$ satisfying the conditions of Definition 11.4, we have:

\begin{tabular}{r|c|c|c} 
& $a+h(a) \leq x$ & $x \leq a+h(a) \leq b$ & $b \leq a+h(a)$ \\
\hline $\mathbb{E}_{x}\left[e^{-q T_{b,+}} ; T_{b,+} \leq \min \left(\tau_{\widehat{d}(\cdot)}, T_{a,-}\right)\right]=$ & $U b D_{q, \widehat{d}(\cdot)}^{b}(x)$ & $\bar{\Psi}_{q}^{a+h(a)}(x, a) U b D_{q, \widehat{d}(\cdot)}^{b}(a+h(a))$ & $\bar{\Psi}_{q}^{b}(x, a)$ \\
\hline $\mathbb{E}_{x}\left[e^{-q T_{a,-}+\theta\left(X_{\left.T_{a,-}-a\right)} ; T_{a,-} \leq \min \left(\tau_{\widehat{d}(\cdot)}, T_{b,+}\right)\right]=}\right.$ & 0 & $\Psi_{q, \theta}^{a+h(a)}(x, a)$ & $\Psi_{q, \theta}^{b}(x, a)$ \\
\hline $\mathbb{E}_{x}\left[e^{-q \tau_{\widehat{d}(\cdot)}-\theta\left(Y_{\tau_{\widehat{d}(\cdot)}}-d\right)} ; \tau_{\widehat{d}(\cdot)} \leq \min \left(T_{b,+}, T_{a,-}\right)\right]=$ & $D b U_{q, \theta, \widehat{d}(\cdot)}^{b}(x)$ & $\bar{\Psi}_{q}^{a+h(a)}(x, a) D b U_{q, \theta, \widehat{d}(\cdot)}^{b}(a+h(a))$ & 0
\end{tabular}

\subsection{First passage theory for upwards skip-free Markovian processes: $\nu$ and $\delta$ replace $W, Z$}

In this section, we review the functions $\nu_{q}, \delta_{q, \theta}$, essentially differential versions of the scale functions $W_{q}, Z_{q}$ of spectrally negative Lévy theory, which serve to extend the spectrally negative Lévy theory to the spectrally negative Markov case. They were first constructed in [95, 100], via an "infinitesimal decomposition" approach into two sided infinitesimal exit problems for $X_{t}$ out of intervals $[x-d, x+\epsilon]$. It was later observed in [30] that using intervals $[x-d(x), x+\epsilon]$ allows extending this to the framework of generalized drawdown /Azema-Yor times - see Figure 8 . 

(1.4):

The key step is assuming the existence of differential versions of the ruin and survival probabilities (1.3),

Assumption 11.7. For all $q, \theta \geq 0$ and $y \leq x$ fixed, assume that $\bar{\Psi}_{q}^{b}(x, y)$ and $\Psi_{q, \theta}^{b}(x, y)$ are differentiable in $b$ at $b=x$, and in particular that the following limits exist:

$$
\nu_{q}(x, y):=\lim _{\varepsilon \downarrow 0} \frac{1-\bar{\Psi}_{q}^{x+\varepsilon}(x, y)}{\varepsilon} \quad \text { (total infinitesimal hazard rate) }
$$

and

$$
\delta_{q, \theta}(x, y):=\lim _{\varepsilon \downarrow 0} \frac{\Psi_{q, \theta}^{x+\varepsilon}(x, y)}{\varepsilon} \quad \text { (infinitesimal spatial killing rate) }
$$

Remark 11.8. It turns out that everything reduces to the differentiability of the two-sided ruin and survival probabilities as functions of the upper limit. Informally, we may say that the pillar of first passage theory for spectrally negative Markov processes is proving the existence of $\nu, \delta$.

Remark 11.9. In the spectrally negative Lévy case (1.3), (1.4) imply that $\nu_{q}(x, y)=\frac{W_{q}^{\prime}(x-y)}{W_{q}(x-y)}=\nu_{q}(x-y)$, and $\delta_{q, \theta}(x, y)=\delta_{q, \theta}(x-y)$ with $\delta_{q, \theta}(d)=\nu_{q}(d)\left(Z_{q}(d, \theta)-W_{q}(d) \frac{Z_{q}^{\prime}(d, \theta)}{W_{q}^{\prime}(d)}\right)=\nu_{q}(d) \widetilde{\delta}_{q, \theta}(d)($ see $(6.17))$.

A necessary condition for Assumption 11.7 to hold is that,

$$
\tau_{x}^{+}=0 \text { and } X_{\tau_{x}^{+}}=x, \mathbb{P}_{x^{-}} \text {a.s. for all } x \in \mathbb{R} .
$$

In other words, $X$ must be upward regular ${ }^{37}$ and upward creeping at every $x \in \mathbb{R}$. Assumption 11.7 holds for processes $X$ that are upward skip-free.

Assuming the existence of the limits in Assumption 11.7, ([100], Eq. (3.2), Thm. 3.1, Cor. 3.1) show how to compute the first passage functions from their differential versions. The extension of this result with generalized drawdown times is ([30], Thm. 1):

Proposition 11.10. Consider a Markov process X such that Assumption 11.7 holds. Assume d( $\cdot$ ) satisfies the conditions of Definition 11.4, and $q, \theta \geq 0, b \in \mathbb{R}$.

A) The "upper first passage" function (11.12) is given by

$$
U b D(x)=U b D_{q, \widehat{d}(\cdot)}^{b}(x)=e^{-\int_{x}^{b} \nu_{q}(s, \widehat{d}(s)) \mathrm{d} s},
$$

and satisfies the $O D E$

$$
U b D^{\prime}(y)-\nu_{q}(y, \widehat{d}(y)) U b D(y)=0, \quad U b D(b)=1,
$$

\footnotetext{
${ }^{37} \mathrm{~A}$ process is called upward regular if $P_{y}\left(T_{x,+}<\infty\right)>0$, for all $y<x \in \mathbb{R}$.
} 
B) The "lower first passage" function (11.13) is given by

$$
D b U(x)=D b U_{q, \theta, \widehat{d}(\cdot)}^{b}(x)=\int_{x}^{b} e^{-\int_{x}^{y} \nu_{q}(s, \widehat{d}(s)) \mathrm{d} s} \nu_{q}(y, \widehat{d}(y)) \delta_{q, \theta}(y, \widehat{d}(y)) \mathrm{d} y,
$$

and satisfies the $O D E$

$$
D b U^{\prime}(y)-\nu_{q}(y, \widehat{d}(y)) D b U(y)+\delta_{q, \theta}(y, \widehat{d}(y))=0, \quad D b U(b)=0 .
$$

Proof. See (3.5) in [100] for the case $l(x)=x-d$, and [30] for the general case.

Remark 11.11. We view differential equations like (11.17), (11.19) as the fundamental object of spectrally negative first passage theory, due to their probabilistic interpretation as Kolmogorov equations of the upward ladder process with excised negative excursions.

Remark 11.12. In the spectrally negative Lévy case, (11.16) reduces by using (3.6) to

$$
U b D_{q, \widehat{d}(\cdot)}^{b}(x)=e^{-\int_{x}^{b} \nu_{q}(s, \widehat{d}(s)) \mathrm{d} s}=e^{-\int_{x}^{b} \frac{W_{q}^{\prime}(d(s))}{W_{q}(d(s))} \mathrm{d} s}
$$

and (11.18) becomes

$$
\begin{aligned}
D b U_{q, \theta, \widehat{d}(\cdot)}^{b}(x) & =\int_{x}^{b} e^{-\int_{x}^{y} \nu_{q}(d(s)) \mathrm{d} s} \nu_{q}(d(y)) \widetilde{\delta}_{q, \theta}(d(y)) \mathrm{d} y \\
& =\int_{x}^{b} e^{-\int_{x}^{y} \frac{W_{q}^{\prime}(d(s))}{W_{q}(d(s))} \mathrm{d} s} \frac{W_{q}^{\prime}(d(y))}{W_{q}(d(y))}\left(Z_{q}(d(y), \theta)-W_{q}(d(y)) \frac{Z_{q}^{\prime}(d(y), \theta)}{W_{q}^{\prime}(d(y))}\right) \mathrm{d} y .
\end{aligned}
$$

Furthermore, if we have classic drawdown $d(s)=d \geq 0$, then we obtain (11.7) and (11.9)

$$
\begin{aligned}
U b D_{q, \widehat{d}(\cdot)}^{b}(x) & =e^{-(b-x) \frac{W_{q}^{\prime}(d)}{W_{q}(d)}}=U b D_{q, d}^{b}(x), \\
D b U_{q, \theta, \widehat{d}(\cdot)}^{b}(x) & =\int_{x}^{b} e^{-\int_{x}^{y} \frac{W_{q}^{\prime}(d)}{W_{q}(d)} \mathrm{d} s} \frac{W_{q}^{\prime}(d)}{W_{q}(d)} \widetilde{\delta}_{q, \theta}(d) \mathrm{d} y=\left(1-e^{-(b-x) \frac{W_{q}^{\prime}(d)}{W_{q}(d)}}\right) \widetilde{\delta}_{q, \theta}(d)=D b U_{q, \theta, d}^{b}(x) .
\end{aligned}
$$

We may also express Proposition 11.10 in terms of a generalized $W, Z$ basis.

Remark 11.13. (1) Introducing

$$
W_{q, d(\cdot)}(x, a):=e^{\int_{a}^{x} \nu_{q}(s, \widehat{d}(s)) \mathrm{d} s} \Leftrightarrow \nu_{q}(x, \widehat{d}(x))=\frac{W_{q, d(\cdot)}^{\prime}(x)}{W_{q, d(\cdot)}(x)},
$$

for some arbitrary $a \leq x$, we may rewrite (11.16) as

$$
U b D_{q, \widehat{d}(\cdot)}^{b}(x)=\frac{W_{q, d(\cdot)}(x, a)}{W_{q, d(\cdot)}(b, a)} .
$$

(2) We may rewrite (11.18) in an alternative form

$$
D b U_{q, \theta, \widehat{d}(\cdot)}^{b}(x)=Z_{q, d(\cdot)}(x, \theta)-\frac{W_{q, d(\cdot)}(x)}{W_{q, d(\cdot)}(b)} Z_{q, d(\cdot)}(b, \theta) .
$$


where we put $\widetilde{\delta}_{q, \theta}(x, y):=\frac{\delta_{q, \theta}(x, y)}{\nu_{q}(x, y)}$ and

$$
\begin{aligned}
Z_{q, d(\cdot)}(x, \theta) & :=\widetilde{\delta}_{q, \theta}(x, \widehat{d}(x))+W_{q, d(\cdot)}(x) \int_{x}^{\infty} \frac{\widetilde{\delta}_{q, \theta}^{\prime}(s, \widehat{d}(s))}{W_{q, d(\cdot)}(s)} \mathrm{d} s \\
& \Leftrightarrow \frac{Z_{q, d(\cdot)}^{\prime}(x, \theta)}{W_{q, d(\cdot)}^{\prime}(x)}=\int_{x}^{\infty} \frac{\widetilde{\delta}_{q, \theta}^{\prime}(s, \widehat{d}(s))}{W_{q, d(\cdot)}(s)} \mathrm{d} s \\
& \Leftrightarrow \widetilde{\delta}_{q, \theta}^{\prime}(x, \widehat{d}(x))=-W_{q, d(\cdot)}(x)\left(\frac{Z_{q, d(\cdot)}^{\prime}(x, \theta)}{W_{q, d(\cdot)}^{\prime}(x)}\right)^{\prime}
\end{aligned}
$$

Remark 11.14. Note that while $\nu, \delta$ are just functions of two variables, in the drawdown framework $W$ and $Z$ are functionals of the initial position and of the drawdown function $d(\cdot)$.

Proof. B) It may be checked that substituting $Z_{q, d(\cdot)}(x, \theta)$ given by the first equality in (11.23) into (11.22) yields $D b U(x)=\widetilde{\delta}_{q, \theta}(x, \widehat{d}(x))-\widetilde{\delta}_{q, \theta}(b, \widehat{d}(b)) \frac{W_{q, d(\cdot)}(x)}{W_{q, d(\cdot)}(b)}+W_{q, d(\cdot)}(x) \int_{x}^{b} \frac{\widetilde{\delta}_{q, \theta}^{\prime}(s, \widehat{d}(s))}{W_{q, d(\cdot)}(s)} \mathrm{d} s$; but this is just an alternative way to express the solution of the ODE (11.19), obtained by an integration by parts.

Remark 11.15. With classic first passage stopping $\widehat{d}(x)=a$, and we obtain

$$
\bar{\Psi}_{q}^{b}(x, a)=\frac{W_{q}(x, a)}{W_{q}(b, a)}, \quad \Psi_{q, \theta}^{b}(x, a)=Z_{q}(x, a, \theta)-W_{q}(x, a) \frac{Z_{q}(b, a, \theta)}{W_{q}(b, a)}
$$

with scale functions involving now just the variable $a$ (the non-smooth first passage end), which reduce to the classic Lévy formulas upon replacing $(x, a)$ by $x-a$.

Example 11.16. With fixed drawdown stopping $d(x)=d$, in the Lévy spectrally negative case, it follows that $\nu_{q}(d)=\frac{W_{q}^{\prime}(d)}{W_{q}(d)} \Leftrightarrow W_{q, d}(x)=e^{-x \nu_{q}(d)}$. We recover also the simple structure of the parameter $\delta_{q, \theta}([100]$, Ex. 3.1):

$$
\delta_{q, \theta}(d)=Z_{q}(d, \theta) \nu_{q}(d)-Z_{q}^{\prime}(d, \theta)=\frac{W_{q}^{\prime}(d)}{W_{q}(d)} \widetilde{\delta}_{q, \theta}(d)
$$

with $\widetilde{\delta}_{q, \theta}(d)=Z_{q}(d, \theta)-\frac{W_{q}(d)}{W_{q}^{\prime}(d)} Z_{q}^{\prime}(d, \theta)$, and (11.23) becomes

$$
Z_{q, \theta, d}(x)=\frac{e^{x \nu_{q}(d)}+\widetilde{\delta}_{q, \theta}(d)}{1+\widetilde{\delta}_{q, \theta}(d)}
$$

Remark 11.17. Recall now that in the Lévy context, the second scale function $Z[22,78,125]$ may also be defined via the solution of the non-smooth total discounted "regulation" /capital injections problem.

Let $X_{t}^{[d(\cdot)}=X_{t}+L_{t}$ denote the process $X_{t}$ modified by Skorokhod reflection at $d(\cdot)$, and let $\mathbb{E}_{x}^{[d(\cdot)}$ denote expectation for this process and let $T_{b}^{[d(\cdot)}$ denote the first passage to $b$ of $X_{t}^{[d(\cdot)}$.

It may be checked by Ivanovs-Palmowski proof of Theorem 6.3 (see Rem. 6.4) that this keeps being true when generalized drawdown reflection at $d(\cdot)$ replaces reflection at 0 , i.e. that the relation (11.22) is still 
equivalent to

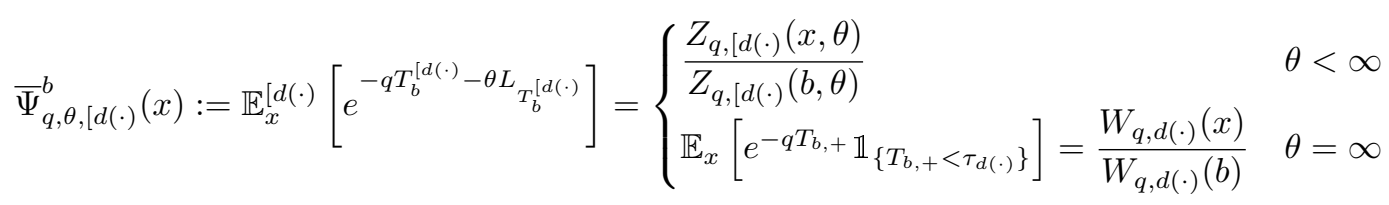

\subsection{Optimal dividends problem with generalized drawdowns}

Let $T_{\widehat{d}(\cdot)}=\tau_{\widehat{d}(\cdot)} \wedge T_{a,-}$ denote the first passage time either below $a$, or below the drawdown boundary for the process $X_{t}^{b]}$ reflected at $b$ with regulator $U_{t}$. One can consider the extension of de Finetti's optimal dividend problem (6.1)

$$
V^{b]}(x)=V_{q, \widehat{d}(\cdot)}^{b]}(x):=\mathbb{E}_{x}\left[\int_{0}^{T_{\widehat{d}(\cdot)}} e^{-q t} \mathrm{~d} U_{t}\right]
$$

where $V$ depends now also on the function $\widehat{d}(\cdot){ }^{38}$

By the strong Markov property, it holds that

$$
V^{b]}(x)=\mathbb{E}_{x}\left[e^{-q T_{b,+}} ; T_{b,+} \leq \min \left(\tau_{\widehat{d}(\cdot)}, T_{a,-}\right)\right] v(b), \quad v(b)=V_{q, \widehat{d}(\cdot)}^{b]}(b)=\mathbb{E}_{b}\left[\int_{0}^{T_{\widehat{d}(\cdot)}} e^{-q t} \mathrm{~d} U_{t}^{b]}\right] .
$$

Remark 11.18. The function $v(b)$ represents the expected discounted time until killing for the reflected process, when starting from $b$. This equals the time the process reflected at $b$ spends at point $(b, 0)$ in Figure 8 , before a downward excursion beyond $\widehat{d}(b)$ kills the process. Furthermore, this time is exponential with parameter $\nu_{q}(b, \widehat{d}(b))$ (as a consequence of the fact that the drawdown process away from a running maximum is Markovian and the corresponding process of upward excursions is Poisson, just as in the Lévy case). Thus, the expectation is the reciprocal of $\nu_{q}(b, \widehat{d}(b))$, and

$$
v(b)=\nu_{q}(b, \widehat{d}(b))^{-1}=\frac{W_{q, d(\cdot)}(b)}{W_{q, d(\cdot)}^{\prime}(b)} .
$$

Remark 11.19. By (11.16), (11.29) we arrive finally to an explicit formula for $V^{b]}(x)$ :

$$
V^{b]}(x)=\frac{e^{-\int_{x}^{b} \nu_{q}(y, \widehat{d}(y)) \mathrm{d} s}}{\nu_{q}(b, \widehat{d}(b))}
$$

expressing the expected dividends in terms of $\nu_{q}(y, \widehat{d}(y))$. Note that in the Lévy case the equation (11.30) simplifies to:

$$
V^{b]}(x)=\frac{W_{q}(d(x))}{W_{q}(d(b))} \nu_{q}(d(b))^{-1}
$$

(using $x-\widehat{d}(x)=d(x)$ ), which checks with Lemmas 3.1-3.2 in [149].

The problem of choosing a drawdown boundary to optimize dividends in (11.30) is tackled in [16] via Pontryaghin's maximum principle. The result depends of course of the process considered, but it always must

\footnotetext{
${ }^{38}$ This definition assumes that the initial point satisfies $X_{0}=\bar{X}_{0}=x$, i.e. that the starting point is on the $x$ axis in Figure 8 .
} 
use one of two types of segments: "de Finetti segments" of maximal slope, of direction $\left(\widehat{d^{\prime}}(s), d^{\prime}(s)=(0,1)\right.$ and segments along which the equation

$$
\partial_{2} \nu_{q}(s, \widehat{d}(s))=\text { const }
$$

is satisfied.

For spectrally negative Lévy process and affine drawdowns $d(x)=(1-\xi) x+d, \widehat{d}(x)=\xi x-d$, $h(x)=d(x) / \xi$, the exit functions and $v(b)$ in (11.29) are simpler:

$$
\begin{aligned}
& W_{q, d(\cdot)}(x)=\left(W_{q}(d(x))\right)^{\frac{1}{1-\xi}}, \quad U b D_{q}^{b}(x, \widehat{d}(\cdot))=\left(\frac{W_{q}(d(x))}{W_{q}(d(b))}\right)^{\frac{1}{1-\xi}}, \\
& \nu_{q}(x, \widehat{d}(x))=\frac{1}{W_{q, d(\cdot)}(x)} \frac{\mathrm{d} W_{q, d(\cdot)}(x)}{\mathrm{d} x}=\frac{W_{q}^{\prime}(d(x))}{W_{q}(d(x))}, \quad v(b)=\frac{W_{q}(d(b))}{W_{q}^{\prime}(d(b))}
\end{aligned}
$$

see Theorem 1.1 in [34], with tax parameter $\gamma=0$, and Remark 7 in [34], with tax parameter $\gamma=1$.

We may obtain in this case a more precise version of Proposition 11.6. Note first that when $a+h(a)>b$, the drawdown constraint is invisible. The value function (11.27) is therefore $\frac{W_{q}(x-a)}{W_{q}^{\prime}(b-a)}$ (which can be maximized by minimizing $b \mapsto W_{q}^{\prime}(b)$ - see Sect. 9.1).

When $a+h(a) \leq b$, combining the discounted probability of reaching $b$ and the value $v(b)$ yields:

Proposition 11.20. Consider a spectrally negative Lévy process $X$ with three times differentiable scale function $W_{q}$. Assume $d(x):=(1-\xi) x+d$, where $d \geq 0, \xi \leq 1, a \leq x \leq b, a+h(a) \leq b$. Then:

$A)$ the expected discounted dividends are:

$$
V^{b]}(x)= \begin{cases}\left(\frac{W_{q}(d(x))}{W_{q}(d(b))}\right)^{\frac{1}{1-\xi}} \frac{W_{q}(d(b))}{W_{q}^{\prime}(d(b))}, & a+h(a) \leq x, \\ \frac{W_{q}(x-a)}{W_{q}((h(a))}\left(\frac{W_{q}((h(a))}{W_{q}(d(b))}\right)^{\frac{1}{(1-\xi)}} \frac{W_{q}(d(b))}{W_{q}^{\prime}(d(b))}, & x \leq a+h(a) .\end{cases}
$$

B) The barrier influence function (which must be optimized in b) in the case $a+h(a) \leq x$ is

$$
B I(b, d, \xi)=\frac{W_{q}(d(b))^{1-\frac{1}{1-\xi}}}{W_{q}^{\prime}(d(b))}=\frac{W_{q}(d(b))^{-\frac{\xi}{1-\xi}}}{W_{q}^{\prime}(d(b))} .
$$

The critical points $b^{*}$ for fixed $d, \xi$ satisfy ${ }^{39}$

$$
\frac{W_{q}^{\prime \prime} W_{q}}{\left(W_{q}^{\prime}\right)^{2}}\left(d\left(b^{*}\right)\right)+\frac{\xi}{1-\xi}=0
$$

For local maxima at $b^{*}>0$ to exist, it is necessary that $\frac{W_{q}^{\prime \prime} W_{q}}{\left(W_{q}^{\prime}\right)^{2}}(0)+\frac{\xi}{1-\xi}<0$ and that $\left(W_{q} W_{q}^{\prime} W_{q}^{\prime \prime \prime}+\right.$ $\left.W_{q}^{\prime \prime}\left(W_{q}^{\prime}\right)^{2}-2 W_{q}\left(W_{q}^{\prime \prime}\right)^{2}\right)\left(d\left(b_{*}\right)\right)>0$.

\footnotetext{
${ }^{39}$ When $\xi=d=0$, we recover in the compound Poisson case the equation $W_{q}^{\prime \prime}(b)=0$.
} 
C) The barrier influence function in the case $x \leq a+h(a)$ is

$$
W_{q}(h(a))^{\frac{\xi}{1-\xi}} B I(b, d, \xi) .
$$

Proof. A) The first case, in which barrier $a$ is invisible, holds by Theorem 1.1 in [34] (by plugging there $\gamma=0){ }^{40}$

The second case holds by the strong Markov property. Note that until $\bar{X}_{t}$ visits $a+h(a)$, the upper drawdown barrier is invisible, and the classic formula for smooth passage applies. Subsequently, we are in the first case, with starting point $x=a+h(a)$, applying the first case and using $d(a+h(a))=h(a)$ (see Fig. 8).

B) For the critical points, note that the sign of $-B I^{\prime}$ coincides with that of $\frac{W_{q}^{\prime \prime} W_{q}}{\left(W_{q}^{\prime}\right)^{2}}(d(b))+\frac{\xi}{1-\xi}$, and that $W^{\prime}$ is positive.

Remark 11.21. To compare value functions when $\xi, d$ vary, let us choose the fixed point $x=a=0$. It may be easily checked that for any $\xi=0, d \geq 0 V^{b]}(0)=\frac{W_{q}(d)}{W_{q}^{\prime}\left(b_{0}\right)}$, where $b_{0}$ is the $\operatorname{argmax}$ of $B I(b)$ when $\xi=0$ (using the translation invariance of Lévy processes).

Also, the "de Finetti solution" $\xi=0$ always beats $\xi>0$ at equal $d$, due to the singularity of $B I(b)(11.34)$ at 0 when $\xi>0$, which makes immediate stopping optimal. Since $W_{q}(d)$ is increasing, it follows that without extra constraints, with affine drawdown boundary, the optimal solution is trivially $\left.d=\infty, \xi=0 \Leftrightarrow b^{*}=1, V^{b^{*}}\right](x)=$ $\infty$. Other solutions become thus of interest only under a constraint $d(a) \leq d_{0}$.

Furthermore, $\xi>0$ becomes interesting once an upper bound on the derivative $d^{\prime}(s)$ or on the total "regret/risk area" is placed - see Figure 8.

Let us provide an example.

Example 11.22. Brownian motion Consider Brownian motion with drift $X(t)=\sigma B_{t}+\mu t$ and affine drawdown stopping. The scale function $W_{q}$ is given in (10.1).

Assume that $x \geq a+h(a)=a+\frac{d(a)}{\xi}=\frac{a+d}{\xi}$ so that the barrier influence function is given by (11.34). By Theorem 11.20, the critical point $b^{*}$ satisfies (11.35) which by using (10.4) reduces to

$$
\frac{\xi}{1-\xi} \frac{\sigma^{2}}{2}\left(\nu_{q}^{\prime}\left(d\left(b^{*}\right)\right)\right)^{2}-\mu \nu_{q}\left(d\left(b^{*}\right)\right)+q=0
$$

Solving the quadratic equation implies that $b^{*}$ satisfies

$$
\frac{\mu}{2 q}+\sqrt{\left(\frac{\mu}{2 q}\right)^{2}-\frac{\sigma^{2} \xi}{2 q(1-\xi)}} \nu_{q}\left(d\left(b^{*}\right)\right)=1,
$$

which reduces when $\xi=0$ to $(10.5)$.

\section{Chronology}

A) Ruin theory for the Cramér-Lundberg or compound Poisson risk model was born in Lundberg's treaty [109].

B) The extension to the Lévy case was achieved in the landmark paper "Problem of destruction and resolvent of a terminating process with independent increments", where the formula

$$
\bar{\Psi}_{q}^{b]}(x, a)=\mathbb{E}_{x}\left[e^{-q T_{b,+}} 1_{\left\{T_{b,+}<T_{0}\right\}}\right]=\frac{W_{q}(x)}{W_{q}(b)}
$$

\footnotetext{
${ }^{40}$ Note that the limiting case $\xi=1$ is consistent by L'Hospital's theorem with our previous $U b D_{q}^{b}(x, d)$ defined in $(11.7)$.
} 
for the "smooth" two-sided exit problem (TSE) ([142], Thm. 3) is provided. ${ }^{41}$ The Laplace transform of $W_{q}$ was computed in (33) from [142]. Also, Theorem 2 from [142] provided the formula of the resolvent density for the process killed outside an interval $[a, b] .{ }^{42}$

$$
u_{q}(x, y)=\frac{W_{q}(x-a)}{W_{q}(b-a)} W_{q}(b-y)-W_{q}(x-y)
$$

C) $[42,(4)-(7)]$ introduced the notation $W_{q}$ and the name scale function for spectrally negative Lévy processes. The central object of the paper is now $W_{q}$ (instead of Suprun's resolvent). Probabilistic proofs of other problems are provided, by reducing them to smooth TSE. The non-smooth two-sided first passage problem is solved in Corollary 1 from [42], and Theorem 2 from [42] determined the decay parameter $\lambda$ of the process killed upon exiting an interval, and showed that the quasi-stationary distribution is $W_{-\lambda}$. The subsequent landmark textbook [43] offers a comprehensive treatment of Lévy processes, including the beautiful excursion theory.

D) A first treatment of the optimal discounted dividends problem in the classical compound Poisson model can be found in Section 6.4 of Buhlmann (1970) [49]. The resulting formula $\frac{W_{q}(b)}{W_{q}^{\prime}(b)}$ for dividends at $b$, when starting from $b$, is a consequence of the fact that the discounted dividends have an exponential law of rate $\frac{W_{q}^{\prime}(b)}{W_{q}(b)}$.

E) [111] studies the Gerber-Shiu function (a generalization of the ruin probability) for a compound Poisson process with a constant barrier and discovers the "dividends-penalty" identity connecting it to the scale function, denoted by $h$, and to the Gerber-Shiu function without barrier.

F) [22] introduced the second scale function $Z_{q}$, initially for relating to $W_{q}$ the solution of the ruin problem $\Psi_{q}(x):=\mathbb{E}_{x}\left[e^{-q T_{0}} \mathbb{1}_{\left\{T_{0}<\infty\right\}}\right]=Z_{q}(x)-W_{q}(x) \frac{q}{\Phi_{q}}$. A case could be made for using $\Psi_{q}(x)$ rather than $Z_{q}(x)$ as the second "alphabet letter" in first passage formulas. In fact, the former, being bounded, is more convenient to compute numerically. However, it turned out that $Z_{q}(x)$ leads often to simpler results and proofs, due to the fact that $e^{-q t} Z_{q}\left(X_{t}\right)$ is a martingale ([22], Rem. 5), [119].

G) $[124,125]$ solved in terms of $W, Z$ several first passage problems for reflected processes.

H) [153] remarks that previous excursion theory proofs can often be replaced by simple applications of the strong Markov property, and of " $\epsilon$ approximation" arguments in the non compound Poisson case.

I) [94] provided a comprehensive textbook on Lévy processes and applications.

J) [86] solved the TSE for refracted processes (which are skip-free, but not Lévy), in terms of extensions of $W$ and $Z$.

K) $[32,78]$ introduced the two variables extension $Z_{q}(x, \theta)$, which is useful for example for computing the Gerber-Shiu function $\Psi_{q, \theta}^{b}(x):=\mathbb{E}_{x}\left[e^{-q T_{0}+\theta X_{T_{0}}} \mathbb{1}_{\left\{T_{0}<T_{b,+}\right\}}\right]=Z_{q}(x, \theta)-\frac{W_{q}(x)}{W_{q}(b)} Z_{q}(b, \theta)$ see Theorem $\left.6.5 \mathrm{~A}\right)$. The first paper showed also that this function was the unique "smooth" $q$-harmonic extension of $e^{x \theta}, x \leq 0$.

L) $[78,79]$ showed that the known formulas on spectrally negative Lévy processes apply for spectrally negative Markov additive processes.

M) $[12,20,33,40,115]$ ibidem for exponential Parisian processes.

N) $[104,114,146]$ ibidem for Omega models (processes with state dependent killing).

O) [19] ibidem for skip-free discrete state-space random walks.

P) $[145,147]$ ibidem for positive self similar Markov processes with one-sided jumps.

Q) $[16,28,30,100]$ initiate the study of time-homogeneous strong Markov processes with one-sided jumps.

\footnotetext{
${ }^{41}$ Informally, $W_{q}$ may be viewed as an analog of the transfer function for discrete systems.

${ }^{42}$ Under the Cramér-Lundberg risk model, [59] derived independently the particular case $q=0$ of the resolvent formula - see also Gerber and Shiu [72, (6.5-6.6)], who extend Dickson's resolvent formula to $q>0$.
} 


\section{List OF NOTATIONS}

\begin{tabular}{|c|c|}
\hline$T_{a,-}, T_{b,+}, \tau_{d(\cdot)}, \underline{\tau}_{d(\cdot)}, T_{a,-}^{b]}, T_{b,+}^{a a}$ & $\begin{array}{l}\text { times of first passage (1.2), drawdown, draw- } \\
\text { up (1.10), first passage with reflection (4.3), } \\
(6.5)\end{array}$ \\
\hline $\bar{\Psi}_{q}^{b}(x, a)=\mathbb{E}_{x}\left[e^{-q T_{b,+}} \mathbb{1}_{\left\{T_{b,+}<T_{a,-}\right\}}\right]=\frac{W_{q}(x-a)}{W_{q}(b-a)}$ & survival probability $(1.3),(3.5)$ \\
\hline$\Psi_{q, \theta}^{b}(x, a)=\mathbb{E}_{x}\left[e^{-q T_{a,-}+\theta\left(X_{\left.T_{a,-}-a\right)} \mathbb{1}_{\left\{T_{a,-}<T_{b,+}\right\}}\right]}\right.$ & ruin probability $(1.4),(4.1)$ \\
\hline$\underline{X}_{t}=\inf _{0 \leq s \leq t} X_{s}, \quad \bar{X}_{t}=\sup _{0 \leq s \leq t} X_{s}$, & infimum and supremum processes (1.7) \\
\hline$L_{t}^{\mid a}=-\left(\underline{X}_{t}-a\right)_{-}, \quad U_{t}=U_{t}^{b]}=\left(\bar{X}_{t}-b\right)_{+}$ & minimal "Skorokhod regulators" (1.7) \\
\hline$X_{t}^{[a}=X_{t}+L_{t}, \quad X_{t}^{b]}=X_{t}-U_{t}$ & regulated processes $(1.7)$ \\
\hline$Y_{t}=\bar{X}_{t}-X_{t}, \quad \widehat{Y}_{t}=X_{t}-\underline{X}_{t}$ & drawdown and draw-up pre \\
\hline$\kappa(\theta), \Phi(q), W_{q}(x), Z_{q}(x, \theta), W_{q, \lambda}(x), Z_{q, \lambda}(x, \theta)$ & $\begin{array}{l}\text { Levy exp. }(2.1) \text {, its invers } \\
(3.1),(5.1),(5.2) \text {, Parisia }\end{array}$ \\
\hline$\nu_{q}(s)=\frac{W_{q}^{\prime}\left(s_{+}\right)}{W_{q}(s)}$ & rate of down excursions larger than $s(3.6)$ \\
\hline$u_{q}(x), u_{q}^{\mid a}(x, y), u_{q}^{[a, b]}(x, y), u_{q}^{[a, b]}(x, y), u_{q}^{[a, b \mid}(x, y)$ & resolvents of free and constrained processes \\
\hline$Z_{q}^{(1)}(x)={\frac{\partial Z_{q}(x, \theta)}{\partial \theta}}_{\theta=0}=\bar{Z}_{q}(x)-\kappa^{\prime}\left(0_{+}\right) \bar{W}_{q}(x)$ & Gerber-Shiu function for $w(x)=x(5.6)$ \\
\hline $\left.\bar{\Psi}_{q, \theta}^{b}(x, a]\right)=\mathbb{E}_{x}^{[a}\left[e^{-q T_{b}^{[a}-\theta L_{T_{b}^{[a}}^{[a}}\right]=\frac{Z_{q}(x-a, \theta)}{Z_{q}(b-a, \theta)}$ & discounted cumulative bailouts (1.11), (6.6) \\
\hline$V^{b]}(x)=\mathbb{E}_{x}^{b]}\left[\int_{0}^{T_{0}^{b]}} e^{-q t} \mathrm{~d} U_{t}\right]=\frac{W_{q}(x)}{W_{q}^{\prime}(b)}$ & expected discounted dividends until $T_{0}^{b]}(6.1)$ \\
\hline$V^{[0, b]}(x)=\mathbb{E}_{x}^{[0, b]}\left[\int_{0}^{\infty} e^{-q t} \mathrm{~d} U_{t}\right]=\frac{Z_{q}(x)}{Z_{q}^{\prime}(b)}$ & $\begin{array}{l}\text { expected discounte } \\
\text { reflection }(6.2)\end{array}$ \\
\hline$V_{w}^{b]}(x)=G_{w}(x)+W_{q}(x) \frac{1-G_{w}^{\prime}(b)}{W_{g}^{\prime}(b)}$ & modified de Finetti objective (9.1) \\
\hline$\delta_{q, \theta}(x, d, s)=\mathbb{E}_{x}\left[e^{-q \tau_{d}-\theta\left(Y_{\tau_{d}}-d\right)} ; \bar{X}_{\tau_{d}} \in \mathrm{d} s\right]$ & ium and $\mathrm{dr}$ \\
\hline$\widetilde{\delta}_{q, \theta}(d)=\mathbb{E}_{x}\left[e^{-q \tau_{d}-\theta\left(Y_{\tau_{d}}-d\right)}\right]=Z_{q}(d, \theta)-W_{q}(d) \frac{Z_{q}^{\prime}(d, \theta)}{W_{q}^{\prime}(d)}, \forall x$ & drawdown function (6.17) \\
\hline$D P_{q, \theta, \vartheta}^{b]}(x):=\mathbb{E}_{x}^{b]}\left[e^{-q T_{0}^{b]}+\theta X_{T_{0}^{b]}}-\vartheta U_{T_{0}^{b]}}}\right], D P_{q, \theta, \vartheta}^{\vdots 0, b]}(x)$ & dividends-penalty functions $(6.19),(8.14)$ \\
\hline$D B_{q, \theta, \vartheta}^{[0, b]}(x)=\mathbb{E}_{x}^{[0, b]}\left[e^{-\vartheta U_{e_{q}}-\theta L_{e_{q}}}\right]$ & function $(6.23)$ \\
\hline $\begin{array}{l}B^{[0, b \mid}(x)=\mathbb{E}_{x}^{[0, b \mid}\left[\int_{0}^{T_{b}^{[0}} e^{-q t} \mathrm{~d} L_{t}\right]=\frac{Z_{q}(x)}{Z_{q}(b)} G_{q}^{B}(b)- \\
G_{q}^{B}(x), G_{q}^{B}(x)=\bar{Z}_{q}(x)+\frac{\kappa^{\prime}\left(0_{+}\right)}{q}, G_{q, \lambda}^{B}(x)=\frac{\lambda}{q+\lambda} G_{q}^{B}(x)\end{array}$ & $\begin{array}{l}\text { expected (Parisian) discounted bailouts until } \\
T_{b}^{[0}(6.26),(6.28),(8.17),(8.18)\end{array}$ \\
\hline$B^{[0, b]}(x)=\mathbb{E}_{x}^{[0, b]}\left[\int_{0}^{\infty} e^{-q t} \mathrm{~d} L_{t}\right]=\frac{Z_{q}(x)}{Z_{q}^{\prime}(b)}\left(G_{q}^{B}\right)^{\prime}(b)-G_{q}^{B}(x)$ & $\begin{array}{l}\text { expected discounted bailouts with double } \\
\text { reflection }(6.27)\end{array}$ \\
\hline$V_{S, k}^{[0, b]}(x)=V^{[0, b]}(x)-k B^{[0, b]}(x)$ & Shreve-Lehoczky-Gaver objective (9.14) \\
\hline $\left.\operatorname{UbD}_{q, \widehat{d}(\cdot)}^{b}(x)=\mathbb{E}_{x} \mid e^{-q T_{b,+}} ; T_{b,+} \leq \tau_{\widehat{d}(\cdot)}\right]$ & up before drawdown (11.12) \\
\hline$D b U_{q, \theta, \widehat{d}(\cdot)}^{b}(x)=\mathbb{E}_{x}\left[e^{-q \tau_{\widehat{d}(\cdot)}-\theta \widetilde{Y}_{\widehat{d}(\cdot)}} ; \tau_{\widehat{d}(\cdot)}<T_{b,+}\right]$ & drawdown before up (11.13) \\
\hline
\end{tabular}




\subsection{A summary of asymptotic relations for spectrally negative Lévy processes}

1. When $\kappa^{\prime}\left(0_{+}\right)>0, \Phi_{q}$ is the asymptotically dominant singularity of $W_{q}(x) \sim \frac{e^{x \Phi_{q}}}{\kappa^{\prime}\left(\Phi_{q}\right)}=\Phi^{\prime}(q) e^{x \Phi_{q}}$ as $x \rightarrow \infty$. Furthermore, by (3.13) $W_{q}(x)=\Phi^{\prime}(q) e^{\Phi_{q} x}-u_{q}(-x)$.

2. Recalling $Z_{q}(x, \theta)=(\kappa(\theta)-q) \int_{0}^{\infty} e^{-\theta y} W_{q}(x+y) \mathrm{d} y(5.1)$, it follows that

$$
\lim _{x \rightarrow \infty} \frac{Z_{q}(x, \theta)}{W_{q}(x)}=(\kappa(\theta)-q) \lim _{x \rightarrow \infty} \int_{0}^{\infty} e^{-\theta y} \frac{W_{q}(x+y)}{W_{q}(x)} \mathrm{d} y=\frac{\kappa(\theta)-q}{\theta-\Phi_{q}} .
$$

When $\theta=0$, this yields

$$
\lim _{x \rightarrow \infty} \frac{Z_{q}(x)}{W_{q}(x)}=\frac{q}{\Phi_{q}}, \quad \lim _{x \rightarrow \infty} \frac{Z_{q}(x)}{Z_{q}(x, \theta)}=\frac{\Phi_{q}-\theta}{q-\kappa(\theta)} \frac{q}{\Phi_{q}} .
$$

3. Recalling $Z_{q}(x, \theta)=\frac{\kappa(\theta)-q}{\theta-\Phi_{q}} W_{q}(x)+\Psi_{q, \theta}(x)(5.1)$, it follows that

$$
\lim _{\theta \rightarrow \infty} Z_{q}(x, \theta) \frac{\theta-\Phi_{q}}{\kappa(\theta)-q}=W_{q}(x)
$$

and

$$
\lim _{\lambda \rightarrow \infty} Z_{q}(x, \Phi(q+\lambda)) \frac{\Phi(q+\lambda)}{\lambda}=\lim _{\lambda \rightarrow \infty} Z_{q}(x, \Phi(q+\lambda)) \frac{\Phi(q+\lambda)-\Phi_{q}}{\lambda}=W_{q}(x)
$$

Acknowledgements. Many thanks to Hansjoerg Albrecher, Ester Frostig, Jevgenijs Ivanovs, Bin Li, Ronnie Loeffen, Zbigniew Palmovski, José-Luis Perez, Martijn Pistorius, Matija Vidmar and Xiaowen Zhou for useful discussions, and for their invaluable contributions to this field. D. Grahovac acknowledges the support of University of Osijek grant ZUP2018-31.

\section{REFERENCES}

[1] H. Albrecher and S. Asmussen, Ruin Probabilities, Vol. 14. World Scientific, Singapore (2010).

[2] H. Albrecher and A. Cani, Risk theory with affine dividend payment strategies, in Number Theory-Diophantine Problems, Uniform Distribution and Applications. Springer, Berlin (2017) 25-60.

[3] H. Albrecher and J. Ivanovs, A risk model with an observer in a Markov environment. Risks 1 (2013) $148-161$.

[4] H. Albrecher and J. Ivanovs, Power identities for Lévy risk models under taxation and capital injections. Stoch. Syst. 4 (2014) $157-172$.

[5] H. Albrecher and J. Ivanovs, Strikingly simple identities relating exit problems for Lévy processes under continuous and Poisson observations. Stoch. Process. Appl. 127 (2017) 643-656.

[6] H. Albrecher and J. Ivanovs, Linking dividends and capital injections-a probabilistic approach. Scand. Actuar. J. 2018 (2018) $76-83$.

[7] H. Albrecher and J. Ivanovs, On the joint distribution of tax payments and capital injections for a Lévy risk model. Probab. Math. Stat. 37 (2018) 219-227.

[8] H. Albrecher, S. Borst, O. Boxma and J. Resing, The tax identity in risk theory - a simple proof and an extension. Insur. Math. Econ. 44 (2009) 304-306.

[9] H. Albrecher, F. Avram and D. Kortschak, On the efficient evaluation of ruin probabilities for completely monotone claim distributions. J. Comput. Appl. Math. 233 (2010) 2724-2736.

[10] H. Albrecher, H.U Gerber and E.S.W. Shiu, The optimal dividend barrier in the Gamma-Omega model. Eur. Actuar. J. 1 (2011) 43-55.

[11] H. Albrecher, F. Avram, C. Constantinescu and J. Ivanovs, The tax identity for Markov additive risk processes. Method. Comput. Appl. Probab. 16 (2014) 245-258.

[12] H. Albrecher, J. Ivanovs and X. Zhou, Exit identities for Lévy processes observed at Poisson arrival times. Bernoulli 22 (2016) 1364-1382. 
[13] S. Asmussen, Applied probability and queues, Vol. 51. Springer, Berlin (2003).

[14] S. Asmussen and T. Rolski, Computational methods in risk theory: a matrix-algorithmic approach. Insur. Math. Econ. 10 (1992) 259-274.

[15] S. Asmussen, F. Avram and M.R. Pistorius, Russian and american put options under exponential phase-type Lévy models. Stoch. Process. Appl. 109 (2004) 79-111.

[16] F. Avram and D. Goreac, A pontryaghin minimum principle approach for the optimization of dividends of spectrally negative markov processes, until a generalized drawdown time. Scand. Actuar. J. 9 (2019) 799-823.

[17] F. Avram and A. Minca, Steps towards a management toolkit for central branch risk networks, using rational approximations and matrix scale functions, in Modern trends in controlled stochastic processes: theory and applications, edited by A.B. Piunovskyi. Luniver Press (2015) 263-285.

[18] F. Avram and A. Minca, On the central management of risk networks. Adv. Appl. Probab. 49 (2017) $221-237$.

[19] F. Avram and M. Vidmar, First passage problems for upwards skip-free random walks via the $\Phi, W, Z$ paradigm. Preprint arXiv:1708.06080 (2017).

[20] F. Avram and X. Zhou, On fluctuation theory for spectrally negative Lévy processes with Parisian reflection below, and applications. Theory Probab. Math. Stat. 95 (2017) 17-40.

[21] F. Avram, T. Chan and M. Usabel, On the valuation of constant barrier options under spectrally one-sided exponential Lévy models and carr's approximation for american puts. Stoch. Process. Appl. 100 (2002) 75-107.

[22] F. Avram, A. Kyprianou and M. Pistorius, Exit problems for spectrally negative Lévy processes and applications to (Canadized) Russian options. Ann. Appl. Probab. 14 (2004) 215-238.

[23] F. Avram, D.-C. Fotso and A. Horváth, On moments based Padé approximations of ruin probabilities. J. Comput. Appl. Math. 235 (2011) 3215-3228.

[24] F. Avram, A. Horvath and M.R. Pistorius, On matrix exponential approximations of the infimum of a spectrally negative Lévy process. Preprint arXiv:1210.2611 (2012).

[25] F. Avram, R. Biard, C. Dutang, S. Loisel and L. Rabehasaina, A survey of some recent results on risk theory. ESAIM: PS 44 (2014) 322-337.

[26] F. Avram, A.D. Banik and A. Horvath, Ruin probabilities by Padé's method: simple moments based mixed exponential approximations (Renyi, De Vylder, Cramér-Lundberg), and high precision approximations with both light and heavy tails. Eur. Actuar. J. 9 (2019) 273-299.

[27] F. Avram, D. Goreac and J.-F. Renaud, The Løkka-Zervos Alternative for a Cramér-Lundberg Process with Exponential Jumps. Risks 7 (2019) 120.

[28] F. Avram, D. Grahovac and C. Vardar-Acar, The $W, Z / \nu, \delta$ paradigm for the first passage of strong markov processes without positive jumps. Risks 7 (2019) 18.

[29] F. Avram, A. Horváth, S. Provost and U. Solon, On the Padé and Laguerre-Tricomi-Weeks Moments Based Approximations of the Scale Function W and of the Optimal Dividends Barrier for Spectrally Negative Lévy Risk Processes. Risks 7 (2019) $273-299$.

[30] F. Avram, B. Li and S. Li, General drawdown of general tax model in a time-homogeneous Markov framework. Preprint arXiv:1810.02079 (2018).

[31] F. Avram, Z. Palmowski and M.R. Pistorius, On the optimal dividend problem for a spectrally negative Lévy process. Ann. Appl. Probab. 17 (2007) 156-180.

[32] F. Avram, Z. Palmowski and M.R. Pistorius, On Gerber-Shiu functions and optimal dividend distribution for a Lévy risk process in the presence of a penalty function. Ann. Appl. Probab. 25 (2015) 1868-1935.

[33] F. Avram, J.L. Pérez and K. Yamazaki, Spectrally negative Lévy processes with Parisian reflection below and classical reflection above. Stoch. Process. Appl. 128 (2018) 255-290.

[34] F. Avram, N.L. Vu and X. Zhou, On taxed spectrally negative Lévy processes with draw-down stopping. Insur. Math. Econ. 76 (2017) 69-74.

[35] P. Azcue and N. Muler, Optimal reinsurance and dividend distribution policies in the Cramér-Lundberg model. Math. Finance 15 (2005) 261-308.

[36] P. Azcue and N. Muler, Stochastic Optimization in Insurance: A Dynamic Programming Approach. Springer, Berlin (2014).

[37] J. Azéma and M. Yor, Une solution simple au probleme de Skorokhod, in Séminaire de probabilités XIII. Springer, Berlin (1979), 90-115.

[38] E.J. Baurdoux, Last exit before an exponential time for spectrally negative Lévy processes. J. Appl. Probab. 46 (2009) 542-558.

[39] E. Bayraktar, A.E. Kyprianou and K. Yamazaki, On optimal dividends in the dual model. ASTIN Bull. J. IAA 43 (2013) 359-372.

[40] E. Baurdoux, J.C. Pardo, J.L. Pérez and J.-F. Renaud, Gerber-Shiu distribution at Parisian ruin for Lévy insurance risk processes. J. Appl. Probab. 53 (2016) 572-584.

[41] E.J. Baurdoux, Z. Palmowski and M.R. Pistorius. On future drawdowns of Lévy processes. Stoch. Process. Appl. 127 (2017) 2679-2698.

[42] J. Bertoin, Exponential decay and ergodicity of completely asymmetric Lévy processes in a finite interval. Ann. Appl. Probab. 7 (1997) 156-169.

[43] J. Bertoin, Lévy processes, Vol. 121. Cambridge University Press, Cambridge (1998).

[44] N.H. Bingham, Continuous branching processes and spectral positivity. Stoch. Process. Appl. 4 (1976) $217-242$. 
[45] O.J. Boxma, A.L. and D. Perry, Threshold strategies for risk processes and their relation to queueing theory. J. Appl. Probab. 48 (2011) 29-38.

[46] E. Boguslavskaya, On optimization of dividend flow for a company in a presence of liquidation value. Available at http://www.boguslavsky.net/fin/dividendflow.pdf (2003).

[47] A.A. Borovkov, Stochastic processes in queueing theory, Vol. 4. Springer Science \& Business Media, Berlin (2012).

[48] P.J. Brockwell, S.I. Resnick and R.L. Tweedie, Storage processes with general release rule and additive inputs. Adv. Appl. Probab. 14 (1982) 392-433.

[49] H. Bühlmann, Mathematical methods in risk theory, Vol. 172. Springer Science \& Business Media, Berlin (2007).

[50] P. Carr, First-order calculus and option pricing. J. Financial Eng. 1 (2014) 1450009.

[51] M.E. Caballero, J.-L.P. Garmendia and G.-U. Bravo, A Lamperti-type representation of continuous-state branching processes with immigration. Ann. Probab. 41 (2013) 1585-1627.

[52] T. Chan, A.E. Kyprianou and M. Savov, Smoothness of scale functions for spectrally negative Lévy processes. Probab. Theory Relat. Fields 150 (2011) 691-708.

[53] C. Cai and B. Li, Occupation times of intervals until last passage times for spectrally negative Lévy processes. J. Theor. Probab. 31 (2018) 2194-2215.

[54] M.E. Caballero, A. Lambert and G.U. Bravo, Proof(s) of the Lamperti representation of continuous-state branching processes. Probab. Surv. 6 (2009) 62-89.

[55] I. Czarna, J.-L. Pérez, T. Rolski and K. Yamazaki, Fluctuation theory for level-dependent Lévy risk processes. Preprint arXiv:1712.00050 (2017).

[56] B. de Finetti. Su un'impostazione alternativa della teoria collettiva del rischio, in Vol. 2 of Transactions of the XVth international congress of Actuaries (1957) 433-443.

[57] F. Dufresne and H.U. Gerber, Risk theory for the compound Poisson process that is perturbed by diffusion. Insur. Math. Econ. 10 (1991) 51-59.

[58] F. Dufresne, H.U. Gerber and Elias S.W. Shiu, Risk theory with the gamma process. ASTIN Bull. 21 (1991) $177-192$.

[59] D.C.M. Dickson, On the distribution of the surplus prior to ruin. Insur. Math. Econ. 11 (1992) $191-207$.

[60] K. Debicki, K.M. Kosiński and M. Mandjes, On the infimum attained by a reflected Lévy process. Queueing Syst. 70 (2012) 23-35.

[61] K. Debicki and M. Mandjes, Queues and Lévy fluctuation theory. Springer, Berlin (2015).

[62] R.A. Doney, Some excursion calculations for spectrally one-sided Lévy processes in Séminaire de Probabilités XXXVIII. Springer, Berlin (2005) 5-15.

[63] R.A. Doney, Fluctuation Theory for Levy Processes: École d'Été de Probabilités de Saint-Flour XXXV-2005. Springer, Berlin (2007).

[64] L. Döring and M. Savov, (Non)Differentiability and asymptotics for potential densities of subordinators. Electron. J. Probab. 16 (2011) 470-503.

[65] L.E. Dubins, L.A. Shepp, A.N. Shiryaev, Optimal stopping rules and maximal inequalities for Bessel processes. Theory Probab. Appl. 38 (1994) 226-261.

[66] D. Dickson and H.R. Waters, Some optimal dividends problems. Astin Bull. 34 (2004) $49-74$.

[67] M. Egami and T. Oryu, An excursion-theoretic approach to regulator's bank reorganization problem. Operat. Res. 63 (2015) $527-539$

[68] H.U. Gerber, Entscheidungskriterien für den zusammengesetzten Poisson-Prozess. Ph.D. thesis, ETH Zurich (1969).

[69] H.U. Gerber, Games of economic survival with discrete-and continuous-income processes. Operat. Res. 20 (1972) $37-45$.

[70] H.U. Gerber, X.S. Lin and H. Yang. A note on the dividends-penalty identity and the optimal dividend barrier. ASTIN Bull. J. IAA 36 (2006) 489-503.

[71] D. Grahovac, Densities of ruin-related quantities in the Cramér-Lundberg model with Pareto claims. Method. Comput. Appl. Probab. 20 (2018) 273-288.

[72] H.U. Gerber and E.S.W. Shiu, On the time value of ruin. North Am. Actuar. J. 2 (1998) 48-72.

[73] H.U. Gerber and E.S.W. Shiu, Optimal dividends: analysis with Brownian motion. North Am. Actuar. J. 8 (2004) 1-20.

[74] H.U. Gerber, E.S.W. Shiu and H. Yang, The Omega model: from bankruptcy to occupation times in the red. Eur. Actuar. J. 2 (2012) 259-272.

[75] C. Hernandez, M. Junca and H. Moreno-Franco. A time of ruin constrained optimal dividend problem for spectrally one-sided Lévy processes. Insur. Math. Econ. 79 (2018) 57-68.

[76] D. Hobson, Optimal stopping of the maximum process: a converse to the results of Peskir. Stoch. Int. J. Probab. Stoch. Process. 79 (2007) 85-102.

[77] M. Huzak, M. Perman, H. Sikic and Z. Vondracek, Ruin probabilities and decompositions for general perturbed risk processes. Ann. Appl. Probab. 7 (2004) 1378-1397.

[78] J. Ivanovs and Z. Palmowski, Occupation densities in solving exit problems for Markov additive processes and their reflections. Stoch. Process. Appl. 122 (2012) 3342-3360.

[79] J. Ivanovs, One-sided Markov additive processes and related exit problems, Ph.D. thesis, Eurandom (2011).

[80] J. Ivanovs, Spectrally-negative Markov additive processes 1.0. Mathematica 8.0 package available from: https://sites.google.com/site/jevgenijsivanovs/files (2013).

[81] J. Ivanovs, Potential measures of one-sided Markov additive processes with reflecting and terminating barriers. J. Appl. Probab. 51 (2014) 1154-1170. 
[82] J. Ivanovs, Sparre Andersen identity and the last passage time. J. Appl. Probab. 53 (2016) 600-605.

[83] M. Jacobsen and A.T. Jensen, Exit times for a class of piecewise exponential Markov processes with two-sided jumps. Stoch. Process. Appl. 117 (2007) 1330-1356.

[84] M. Jeanblanc-Picqué and A.N. Shiryaev, Optimization of the flow of dividends. Russ. Math. Surv. 50 (1995) 257.

[85] A. Kuznetsov, A.E. Kyprianou and V. Rivero, The theory of scale functions for spectrally negative Lévy processes, in Lévy Matters II. Springer, Berlin (2013), 97-186.

[86] A. Kyprianou and R. Loeffen, Refracted Lévy processes. Ann. Inst. Henri Poincaré, Prob. Stat. 46 (2010) $24-44$.

[87] L. Kruk, J. Lehoczky, K. Ramanan and S. Shreve, An explicit formula for the skorokhod map on [0, a]. Ann. Probab. 35 (2007) 1740-1768.

[88] A.E. Kyprianou and Z. Palmowski, A martingale review of some fluctuation theory for spectrally negative Lévy processes, in Séminaire de Probabilités XXXVIII. Springer, Berlin (2005) 16-29.

[89] A. Kyprianou and Z. Palmowski, Fluctuations of spectrally negative Markov additive processes, in Séminaire de probabilités XLI. Springer, Berlin (2008) 121-135.

[90] A. Kyprianou, J.C. Pardo and J.L. Pérez, Occupation times of refracted Lévy processes. J. Theor. Probab. 27 (2014) 12921315.

[91] A.E. Kyprianou and B.A. Surya, Principles of smooth and continuous fit in the determination of endogenous bankruptcy levels. Finance Stoch. 11 (2007) 131-152.

[92] K. Kawazu and S. Watanabe, Branching processes with immigration and related limit theorems. Theory Probab. Appl. 16 (1971) 36-54.

[93] A. Kyprianou, Gerber-Shiu risk theory. Springer Science \& Business Media, Berlin (2013).

[94] A. Kyprianou, Fluctuations of Lévy Processes with Applications: Introductory Lectures. Springer Science \& Business Media, Berlin (2014).

[95] J.P. Lehoczky, Formulas for stopped diffusion processes with stopping times based on the maximum. Ann. Probab. 5 (1977) 601-607.

[96] S. Li, The distribution of the dividend payments in the compound Poisson risk model perturbed by diffusion. Scand. Actuar. J. 2006 (2006) 73-85.

[97] K. Lindensjö and F. Lindskog. Optimal dividends and capital injection under dividend restrictions. Preprint arXiv:1902.06294, (2019).

[98] D. Landriault, B. Li and S. Li, Analysis of a draw-down-based regime-switching Lévy insurance model. Insur. Math. Econ. 60 (2015) 98-107.

[99] D. Landriault, B. Li and H. Zhang, On magnitude, asymptotics and duration of drawdowns for Lévy models. Bernoulli 23 (2017) 432-458.

[100] D. Landriault, B. Li and H. Zhang, A unified approach for drawdown (drawup) of time-homogeneous Markov processes. J. Appl. Probab. 54 (2017) 603-626.

[101] R.L. Loeffen, On optimality of the barrier strategy in de Finetti's dividend problem for spectrally negative Lévy processes. Ann. Appl. Probab. 18 (2008) 1669-1680.

[102] R.L. Loeffen, Stochastic control for spectrally negative Lévy processes. University of Bath, Bath (2008).

[103] S. Loisel, Differentiation of some functionals of risk processes, and optimal reserve allocation. J. Appl. Probab. 42 (2005) 379-392.

[104] B. Li and Z. Palmowski, Fluctuations of Omega-killed spectrally negative Lévy processes. Stoch. Process. Appl. 128 (2018) 3273-3299.

[105] R.L. Loeffen and J.-F. Renaud, De Finetti's optimal dividends problem with an affine penalty function at ruin. Insur. Math. Econ. 46 (2010) 98-108.

[106] D. Landriault, J.-F. Renaud and X. Zhou, Occupation times of spectrally negative Lévy processes with applications. Stoch. Process. Appl. 121 (2011) 2629-2641.

[107] D. Landriault, J.-F. Renaud and X. Zhou, An insurance risk model with Parisian implementation delays. Method. Comput. Appl. Probab. 16 (2014) 583-607.

[108] R.L. Loeffen, J.-F. Renaud and X. Zhou, Occupation times of intervals until first passage times for spectrally negative Lévy processes. Stoch. Process. Appl. 124 (2014) 1408-1435.

[109] F. Lundberg, I. Approximerad framstallning af sannolikhetsfunktionen: II. Aterforsakring af kollektivrisker. Uppsala. 1903.

[110] B. Li, L. Vu and X. Zhou, Exit problems for general draw-down times of spectrally negative Lévy processes. Preprint arXiv:1702.07259 (2017).

[111] X.S. Lin, G.E. Willmot and S. Drekic, The classical risk model with a constant dividend barrier: analysis of the Gerber-Shiu discounted penalty function. Insur. Math. Eco. 33 (2003) 551-566.

[112] Y. Li, C. Yin and X. Zhou, On the last exit times for spectrally negative Lévy processes. J. Appl. Probab. 54 (2017) $474-489$.

[113] A. Løkka and M. Zervos, Optimal dividend and issuance of equity policies in the presence of proportional costs. Insur. Math. Econ. 42 (2008) 954-961.

[114] B. Li and X. Zhou, On weighted occupation times for refracted spectrally negative Lévy processes. J. Math. Anal. Appl. 466 (2018) 215-237.

[115] Y. Li, X. Zhou and N. Zhu, Two-sided discounted potential measures for spectrally negative Lévy processes. Stat. Probab. Lett. 100 (2015) 67-76.

[116] E. Mayerhofer, Three essays on stopping. Preprint arXiv:1909.13050 (2019). 
[117] M.H. Miller and F. Modigliani, Dividend policy, growth, and the valuation of shares. J. Bus. 34 (1961) 411-433.

[118] A. Mijatovic and M.R. Pistorius, On the drawdown of completely asymmetric Lévy processes. Stoch. Process. Appl. 122 (2012) 3812-3836.

[119] L. Nguyen-Ngoc and M. Yor, Some martingales associated to reflected Lévy processes, in Séminaire de probabilités XXXVIII. Springer, Berlin (2005) 42-69.

[120] K. Noba, J.-L. Pérez, K. Yamazaki and K. Yano, On optimal periodic dividend strategies for Lévy risk processes. Insur. Math. Econ. 80 (2018) 29-44.

[121] E.S. Page, Continuous inspection schemes. Biometrika 41 (1954) 100-115.

[122] G. Peskir, Optimal stopping of the maximum process: The maximality principle. Ann. Probab. 26 (1998) $1614-1640$.

[123] P. Picard, On some measures of the severity of ruin in the classical Poisson model. Insur. Math. Econ. 14 (1994) $107-115$.

[124] M.R Pistorius, On doubly reflected completely asymmetric Lévy processes. Stoch. Process. Appl. 107 (2003) $131-143$.

[125] M.R Pistorius, On exit and ergodicity of the spectrally one-sided Lévy process reflected at its infimum. J. Theor. Probab. 17 (2004) 183-220.

[126] M.R. Pistorius, A potential-theoretical review of some exit problems of spectrally negative Lévy processes. Séminaire de Probabilités XXXVIII (2005) 30-41.

[127] M.R. Pistorius, An excursion-theoretical approach to some boundary crossing problems and the Skorokhod embedding for reflected Lévy processes, in Séminaire de Probabilités XL. Springer, Berlin (2007) 287-307.

[128] C. Paroissin and L. Rabehasaina, First and last passage times of spectrally positive Lévy processes with application to reliability. Method. Comput. Appl. Probab. 17 (2015) 351-372.

[129] J.-L. Pérez and K. Yamazaki, On the optimality of periodic barrier strategies for a spectrally positive Lévy process. Insur. Math. Econ. 77 (2017) 1-13.

[130] J.-L. Pérez and K. Yamazaki, Mixed periodic-classical barrier strategies for Lévy risk processes. Risks 6 (2018) 33.

[131] J.-L. Pérez and K. Yamazaki, On the refracted-reflected spectrally negative Lévy processes. Stoch. Process. Appl. 128 (2018) 306-331.

[132] J.-L. Pérez, K. Yamazaki, A. Bensoussan, Optimal periodic replenishment policies for spectrally positive Lévy demand processes. Preprint arXiv:1806.09216 (2018).

[133] L.M. Ricciardi, A.D. Crescenzo, V. Giorno and A.G. Nobile, An outline of theoretical and algorithmic approaches to first passage time problems with applications to biological modeling. Math. Jp. 50 (1999) 247-322.

[134] J.-F. Renaud, On the time spent in the red by a refracted Lévy risk process. J. Appl. Probab. 51 (2014) 1171-1188.

[135] J.-F. Renaud, De finetti's control problem with parisian ruin for spectrally negative Lévy processes. Preprint arXiv:1906.05076 (2019).

[136] T. Rolski, H. Schmidli, V. Schmidt and J. Teugels, Stochastic processes for insurance and finance, volume 505. John Wiley \& Sons, New York (2009).

[137] K.-i. Sato, Lévy processes and infinitely divisible distributions. Cambridge University Press, Cambridge (1999).

[138] N.J. Starreveld, R. Bekker, M. Mandjes, Occupation times of alternating renewal processes with Lévy applications. Preprint arXiv:1602.05131 (2016).

[139] H. Schmidli, Stochastic control in insurance. Springer Science \& Business Media, New York (2007).

[140] S.E. Shreve, J.P. Lehoczky and D.P. Gaver, Optimal consumption for general diffusions with absorbing and reflecting barriers. SIAM J. Control Opt. 22 (1984) 55-75.

[141] L. Shepp and A.N. Shiryaev, The Russian option: reduced regret. Ann. Appl. Probab. 3 (1993) 631-640.

[142] V.N. Suprun, Problem of destruction and resolvent of a terminating process with independent increments. Ukr. Math. J. 28 (1976) 39-51.

[143] A. Shiryaev, P. Xu and X.Y. Zhou, Thou shalt buy and hold. Quant. Finance 8 (2008) 765-776.

[144] H.M. Taylor, A stopped Brownian motion formula. Ann. Probab. 3 (1975) 234-246.

[145] M. Vidmar, Exit problems for positive self-similar Markov processes with one-sided jumps. Preprint arXiv:1807.00486 (2018).

[146] M. Vidmar, First passage upwards for state dependent-killed spectrally negative Lévy processes. Preprint arXiv:1803.04885 (2018).

[147] M. Vidmar, A temporal factorization at the maximum for spectrally negative positive self-similar Markov processes. Preprint arXiv:1805.04036 (2018).

[148] W. Wang, Y. Wang and X. Wu, Dividend and capital injection optimization with transaction cost for spectrally negative Lévy risk processes. Preprint arXiv:1807.11171 (2018).

[149] W. Wang and X. Zhou, General drawdown-based de Finetti optimization for spectrally negative Lévy risk processes. J. Appl. Probab. 55 (2018) 513-542.

[150] K. Yamazaki, Inventory control for spectrally positive Lévy demand processes. Math. Operat. Res. 42 (2016) $212-237$.

[151] C. Yin and Y. Wen, Optimal dividend problem with a terminal value for spectrally positive Lévy processes. Insur. Math. Econ. 53 (2013) 769-773.

[152] Y. Zhao, P. Chen and H. Yang, Optimal periodic dividend and capital injection problem for spectrally positive Lévy processes. Insur. Math. Econ. 74 (2017) 135-146.

[153] X. Zhou, Exit problems for spectrally negative Lévy processes reflected at either the supremum or the infimum. J. Appl. Probab. 44 (2007) 1012-1030.

[154] C. Zhang and R. Wu, Total duration of negative surplus for the compound Poisson process that is perturbed by diffusion. $J$. Appl. Probab. 39 (2002) 517-532. 